\author{
A Dissertation \\ Presented to the Faculty of the Graduate School \\ At the University of Missouri
}

In Partial Fulfillment

Of the Requirements for the Degree

Doctor of Philosophy

\author{
By \\ MONICA L. KEARNEY
}

Dr. John P. Thyfault, Ph.D., Dissertation Supervisor

July 2015 
The undersigned, appointed by the dean of the Graduate School,

Have examined the Dissertation entitled

SHORT-TERM AEROBIC EXERCISE TRAINING IN TYPE 2 DIABETES

\author{
Presented by Monica L. Kearney
}

A candidate for the degree of

Doctor of Philosophy

And hereby certify that, in their opinion, it is worthy of acceptance.

John P. Thyfault, PhD

Jill A. Kanaley, $\mathrm{PhD}$

Heather J. Leidy, PhD

Christopher D. Hardin, PhD

Frank W. Booth, PhD 


\section{DEDICATION}

\section{To my Grandmother, Minerva Ann Ruppert}

(1924-2015)

My grandmother is one whom I can only strive to emulate.

Grandma, thank you for instilling in me an appreciation to move, a spirit to learn, the drive to work hard, and the essence to love. I dedicate this dissertation to you. 


\section{ACKNOWLEDGEMENTS}

This dissertation is the contribution of everyone who has supported and mentored me through this process. To say I am grateful is an understatement.

Foremost, to my advisor and mentor, Dr. John Thyfault, thank you. Thank you for accepting me into your lab. Thank you for allowing me the opportunity to learn techniques in so many areas and to truly understand the value of translational research. Thank you for providing me independence in projects and in learning. Thank you for the countless hours reviewing my writing, critiquing my presentations, and helping me become a stronger grant writer and a better thinker and researcher. Thank you for allowing me to do a dissertation in human subjects even though it was not the easiest path for either of us. Thank you for your encouragement and your support in all aspects of my learning and your guidance in understanding my role in this field. Because of you, I am prepared for the next stage. Thank you for being a true mentor.

To my doctoral and dissertation committee members and other faculty members who have provided intellectual growth, thank you for your insight and your contributions to my learning and to these studies. Dr. Jill Kanaley, Dr. Heather Leidy, Dr. Chris Hardin, Dr. Frank Booth, Dr. Kevin Fritsche, Dr. Elizabeth Parks, and Dr. Tom Thomas, you in particular have each brought a unique perspective to my research that I will carry with me as I continue my career. To each of you and to all of my professors, thank you for fostering a desire for knowledge and for your guidance and instruction.

To Dr. Camila Manrique and Charla Jay, thank you for your medical expertise. Dr. Manrique, thank you for supervising numerous stress tests and for your instrumental 
role as study physician. Charla, thank you for operating as an irreplaceable Research Nurse for the mixed meal tests and for the vigilant care of our subjects. Both of you have been invaluable to this study.

To Matt Morris, Grace Meers, and especially Ying Liu, thank you for helping me navigate the worlds of human and animal research and for your patience in teaching me biochemical techniques and helping me with my studies. To Shana Warner, Katie Mikus, Leryn Boyle, Melissa Linden, Tim Heden, Ryan Sheldon, and Terese Zidon,, and to all of my fellow graduate students and peers, thank you for your support, encouragement, and assistance. Sometimes we learn as much from each other as we do in the lab.

Finally, to my family, thank you for accepting everything that has come with this quest and for sharing in my joys and frustrations. You are my ultimate support network, and I recognize all you have done to help me succeed. Especially to Donovan, thank you for being an amazing kiddo and for rolling with this zany life in your first years on Earth. You are truly the light of my existence, and I have become a better person because of you; you make me try harder and do more. And Matthew, the greatest recognition goes to you. I cannot express in one paragraph how much I appreciate your support and sacrifice. I will never forget that moment in our kitchen when I first said, "I think I might want to do a PhD program," and you, without hesitation, simply replied with, "Yes, you should." From this initial encouragement to the many long days and nights, tears and breakdowns, dirty floors and unwashed dishes, and especially for doing more than your share in caring for our son so that I could train and test subjects, I will be forever grateful. It is truly because of you that I have been able to do this. From the bottom of my heart, thank you. 


\section{TABLE OF CONTENTS}

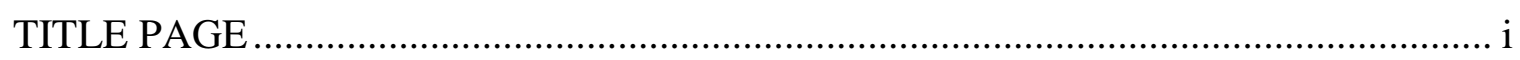

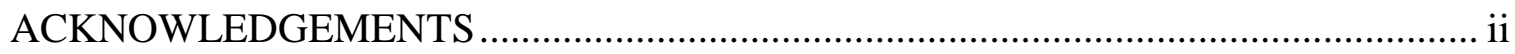

LIST OF TABLES ………………………………........................................... iv

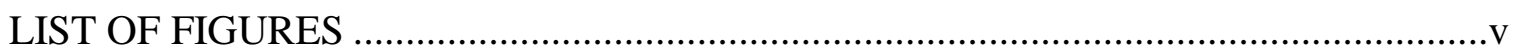

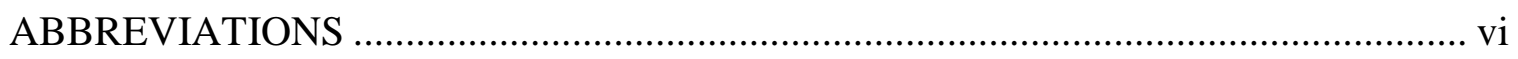

ACADEMIC ABSTRACT ……………......................................................... vii

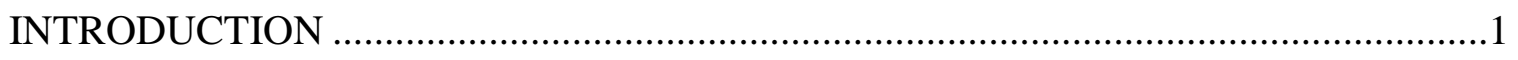

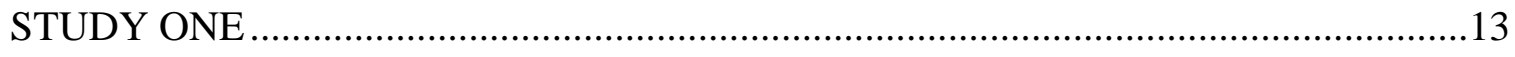

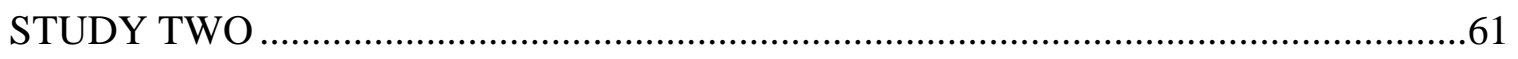

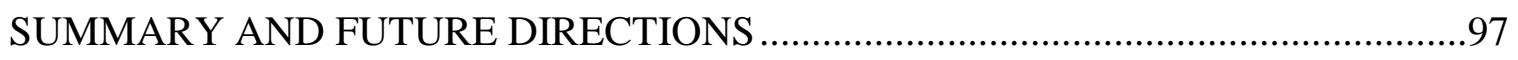

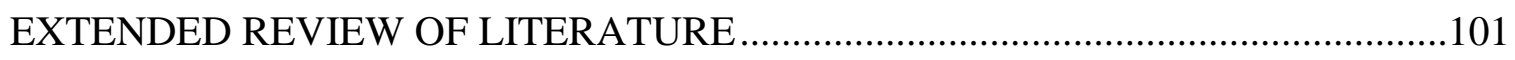

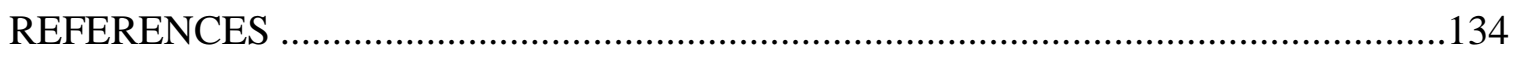

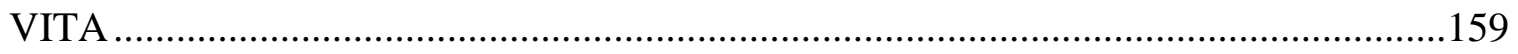




\section{LIST OF TABLES}

\section{Study One}

Table 1. Composition of the breakfast meal for MMTT testing and for the study diet....24

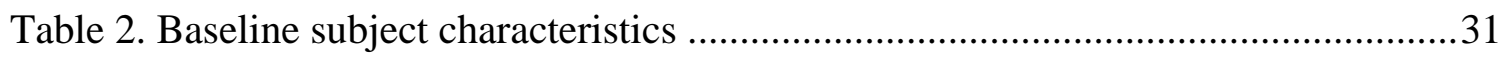

\section{Study Two}

Table 3. Composition of the MMTT meal challenge ................................................. 71

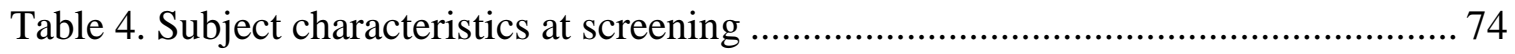




\section{LIST OF FIGURES}

\section{Introduction}

Figure 1. Exercise improves postprandial glycemia through multiple pathways involving increased glucose uptake and decreased glucose output............................................ 5

Figure 2. Schematic representation of the hypothesis for aim 1 ................................. 11

\section{Study One}

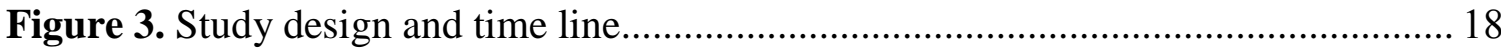

Figure 4. Experimental protocol for the mixed meal tolerance test .............................. 22

Figure 5. Glucose, insulin, and c-peptide responses to a MMTT before (Pre) and after (Post) exercise training

Figure 6. Individual subject responses for glucose, insulin, and c-peptide following a MMTT before (Pre) and after (Post) exercise training 36

Figure 7. Calculations of insulin sensitivity, $\beta$-cell function, and the oral disposition index derived from the MMTT before (Pre) and after (Post) exercise training............... 38

Figure 8. Total glucose rate of appearance $\left(\mathrm{Ra}_{\text {total }}\right)$ was not different between the Pre and Post exercise training conditions

Figure 9. The TTR across the four hour postprandial time course was not different between the Pre and Post exercise training conditions for either $\left[6,6{ }^{2} \mathrm{H}_{2}\right]$ glucose or [U13C6] glucose 


\section{Study Two}

Figure 10. Study design

Figure 11. Glucose responses to MMTTs and OGTTs performed before (Pre) and after (Post) one week of aerobic exercise training in subjects with T2D 76

Figure 12. Insulin responses to MMTTs and OGTTs performed before (Pre) and after (Post) one week of aerobic exercise training in subjects with T2D 78

Figure 13. C-peptide responses to MMTTs and OGTTs performed before (Pre) and after (Post) one week of aerobic exercise training in subjects with T2D. 79

Figure 14. Postprandial insulin clearance following MMTTs and OGTTs performed before (Pre) and after (Post) one week of aerobic exercise training in subjects with T2D

Figure 15. Differences in the Matsuda ISI among MMTTs and OGTTs performed before (Pre) and after (Post) one week of aerobic exercise training in subjects with T2D

\section{Extended Review of Literature}

Figure 16. Illustration of the appearance and disposal of a glucose load in healthy subjects. 104

Figure 17. Plasma glucose and insulin levels during an OGTT (closed circles) and during a MMTT (open circles) 106 


\section{ABBREVIATIONS}

$13 \mathrm{C}$ - uniformly labeled ${ }^{13} \mathrm{C}_{6}$ glucose isotope; $\left[\mathrm{U}^{13} \mathrm{C}_{6}\right]$ glucose

ANOVA - analysis of variance

AUC - area under the curve

BMI - body mass index

bpm - beats per minute

CGMS - continuous glucose monitoring system

d2 glucose - deuterated glucose; $\left[6,6-{ }^{2} \mathrm{H}_{2}\right]$ glucose

DPP-IV - dipeptidyl peptidase IV

DXA - dual energy x-ray absorptiometry

EGP - endogenous glucose production

EX - exercise

GC - glycemic control

GLUT - glucose transporter

HbA1c - hemoglobin A1c

HDL-C - high density lipoprotein cholesterol

HGP - hepatic glucose production

HOMA - homeostatic model assessment

HR - heart rate

IGT - impaired glucose tolerant

GIP - gastric inhibitory polypeptide

GLP-1 - glucagon-like peptide-1 
GLUT4 - glucose transporter 4

iAUC - incremental area under the curve

kcal - kilocalories

LDL-C - low density lipoprotein cholesterol

MMTT - mixed meal tolerance test

mTOR - mammalian target of rapamycin

NADPH - nicotinamide adenine dinucleotide phosphate

OGTT - oral glucose tolerance test

PPG - postprandial glycemia

PPGE - postprandial glycemic excursions

$\mathrm{Ra}_{\text {total }}$ - total glucose rate of appearance

$R a_{\text {oral }}$ - rate of appearance of orally ingested glucose

Rd - rate of disappearance of glucose

SEM - standard error of the mean

SST - serum separator tubes

T2D - type 2 diabetes

$t$ - time

TC - total cholesterol

TTR - tracer to trace ratio

$\mathrm{VO}_{2}$ peak - peak oxygen consumption 


\title{
SHORT-TERM AEROBIC EXERCISE TRAINING \\ IN TYPE 2 DIABETES
}

\author{
Monica L. Kearney \\ John P. Thyfault, PhD, Dissertation Supervisor
}

\begin{abstract}
Type 2 diabetes (T2D) is a worldwide problem, and people with T2D have impaired postprandial glycemic control which contributes to cardiovascular morbidity and mortality. While current pharmaceutical therapies are targeted to reduce glycosylated hemoglobin and may not be effective in improving postprandial glycemia (PPG), exercise is an effective treatment to enhance postprandial glycemic control in patients with T2D. As little as seven days of aerobic exercise has previously been shown to reduce PPG in this population, but the mechanisms by which this occurs currently are not understood. Further, the assessment of postprandial glycemic control generally is done using a nonphysiological glucose overload uncharacteristic of normal human feeding and what the body experiences throughout the day when meals of mixed macronutrients are consumed. Thus, the primary aims of this dissertation were: 1) to identify tissues contributing to changes in glycemic control after short-term exercise training and identify the systemic mechanisms by which exercise improves overall PPG in patients with T2D (Study One), and 2) to determine, in patients with T2D, if a mixed meal tolerance test is a more
\end{abstract}


effective tool for assessing improvements in glycemic control following exercise training than the standard oral glucose tolerance test (Study Two). The data presented here add to current evidence that improved insulin sensitivity is an early adaptation of exercise and short-term exercise training, but we did not see improvements in overall postprandial glucose responses in the sample studied. Further, we show that a mixed meal test is an effective alternative to the oral glucose tolerance test in assessing differences in PPG and the underlying factors which influence PPG. Collectively, when viewed in the presence of the existing literature, these data suggest that while exercise training is ultimately known to enhance postprandial glycemic control, and testing this with a mixed meal test is a viable alternative to the current oral glucose tolerance test, subjects with T2D have variable responses and may not always see improvements in PPG following one week of aerobic exercise training. 


\section{INTRODUCTION}

The burden of type 2 diabetes (T2D) on individuals and society has put it at the forefront of interest, and it is increasing in epidemic proportion. Once a disease thought to occur only later in life, T2D now materializes in an individual's 20 s and 30 s, and the proliferative incidence in children is substantial as well (87). Current estimates indicate that diabetes afflicts $9.3 \%$ of the U.S. population, or over 29 million people (31). Type 2 is by far the most prevalent of the diabetic morbidities, representing greater than $90 \%$ of all diabetes cases, and it is also the segment which is most rapidly rising (31). Diabetes is a huge personal encumbrance, affecting an individual's health, quality of life, and medical expenditures. However, it is also a great social burden due to the high annual and lifetime health care costs of this chronic disease distressing the entire health care system, not just the individuals diagnosed with T2D $(31,41,120)$. Thus, therapies which will effectively treat and manage T2D in individuals, and those which will reduce health care costs, are necessary.

Impairment in glucose regulation is a hallmark of T2D and also affects individuals who are impaired glucose tolerant (IGT) and have pre-diabetes. This lack of glycemic control may be evident in the fasting state, but it is more detrimental following feeding, or the postprandial state, when functional glucose metabolism is critical in avoiding hyperglycemic episodes. Since T2D contributes to cardiovascular events and death in addition to a cacophony of co-morbidities, managing glycemia in these patients is of utmost importance to prolong quality and quantity of life. 
Various methods have been used to assess glycemia, but few truly detect changes in glucose control following a meal. Further, even fewer studies have examined this postprandial glycemic effect in free-living subjects or by using laboratory-based mixed meals, which are more physiologically relevant than standard laboratory methods. Although changes in glycemic control cannot always be detected by the commonly used oral glucose tolerance test (OGTT), other methods, including continuous glucose monitoring (CGMS) and a tolerance test using meals of mixed macronutrient composition (mixed meal tolerance test; MMTT), may be more sensitive in detecting these changes $(108,109)$. Furthermore, exercise, which is perhaps the most cost-effective and advantageous modality to improve glycemic control and particularly postprandial glycemic control, is underutilized due in part to a lack of mechanistic understanding which may contribute to weakness in its acceptance as a first-line therapy in medicine and health care. While it is known that exercise training can improve glycemic control relatively quickly in people with $\mathrm{T} 2 \mathrm{D}(89,109,121)$, how this occurs and what tissues contribute to this improvement remains largely undefined.

\section{Poor glycemic control contributes to morbidity and mortality}

Individuals with T2D have reduced glycemic control (14), and poorly controlled glycemia is linked to cardiovascular disease and other diabetic comorbidities $(40,145$, 154, 172). However, fasting hyperglycemia by itself does not accurately represent glycemic control, and glucose fluctuations in the free-living condition, particularly glucose levels after a meal, or postprandial glycemia (PPG), and the large fluctuations in glucose following meals, or postprandial glycemic excursions (PPGE), may be more 
detrimental to health than stagnant measures of glycemia such as glycosylated hemoglobin (HbA1c). An accumulating body of evidence now suggests that these glucose fluctuations (PPGE) are more tightly correlated to microvascular and macrovascular morbidities and cardiovascular mortality than $\mathrm{HbA} 1 \mathrm{c}$ and measures of fasting glucose $(10,27,28,33)$. Thus, effective therapies are needed which will improve not only stagnant measures of glycemia, but also regulation of glycemia throughout the day and especially after meals, resulting in fewer episodes of both hyperglycemia and hypoglycemia.

\section{Utility of exercise in controlling glycemia in T2D}

Current pharmaceutical therapies for improving glycemic control remain focused on controlling fasting glucose and $\mathrm{HbA} 1 \mathrm{c}$ and are at best moderately effective $(2,168)$. Exercise is the only therapy known to be effective in improving PPG, as available diabetic drugs are designed to lower HbA1c, not PPG or PPGE. Interestingly, increasing physical activity through exercise, one of the most effective therapies for reducing all of these measures, is also one of the most overlooked in the clinical setting. Although clinicians may advise increasing physical activity as a means for weight loss, maintained weight loss can be difficult for overweight, obese, and T2D patients to achieve, and many who lose weight will regain it (92). Even in the absence of weight loss, however, increased physical activity and exercise improve glycemic control (109). Yet, when and how these improvements occur is still very much unknown. This, combined with a general lack of knowledge and adherence from patients, may drive the lack of acceptance of exercise as a first-line treatment from physicians and endocrinologists. If more is 
known on the mechanisms and timing of how exercise improves glycemic control, patients may better understand why exercise is an effective treatment, and physicians may be more apt to prescribe exercise as a primary treatment.

Skeletal muscle insulin resistance, liver insulin resistance, and pancreatic $\beta$-cell dysfunction are all attributes of impaired glycemic control and detrimentally affect PPG $(4,44)$. Ultimately, exercise improves glucose control $(22,46,66,144)$, but certainly not one individual mechanism can be credited. Rather, each of these systems is likely to contribute to an additive or even synergistic effect on glycemic control, and they may do so at different times throughout continued exercise training. As shown in Figure 1, exercise improves PPG in patients with T2D likely through targeting each of these tissues. However, it is not currently known when each of these changes occur and which are affected through short-term exercise training compared to chronic exercise training of longer duration. As such, the improvements seen in glycemic control with a single exercise bout may be mechanistically different from those observed after one week or several weeks of exercise training.

Both an acute exercise bout and short-term exercise training have been shown to improve insulin sensitivity in skeletal muscle $(108,141)$. Though improved skeletal muscle insulin sensitivity is an early outcome likely contributing to improved glycemic control, it is likely not the only early improvement. Changes in endogenous glucose production (EGP), attributed primarily to hepatic glucose production (HGP), also have been shown to occur with exercise training of as little as one week when assessed by hyperinsulinemic euglycemic clamp (89). With improved liver insulin sensitivity, HGP and thus EGP would be more tightly controlled, further reducing glycemic fluctuations. 


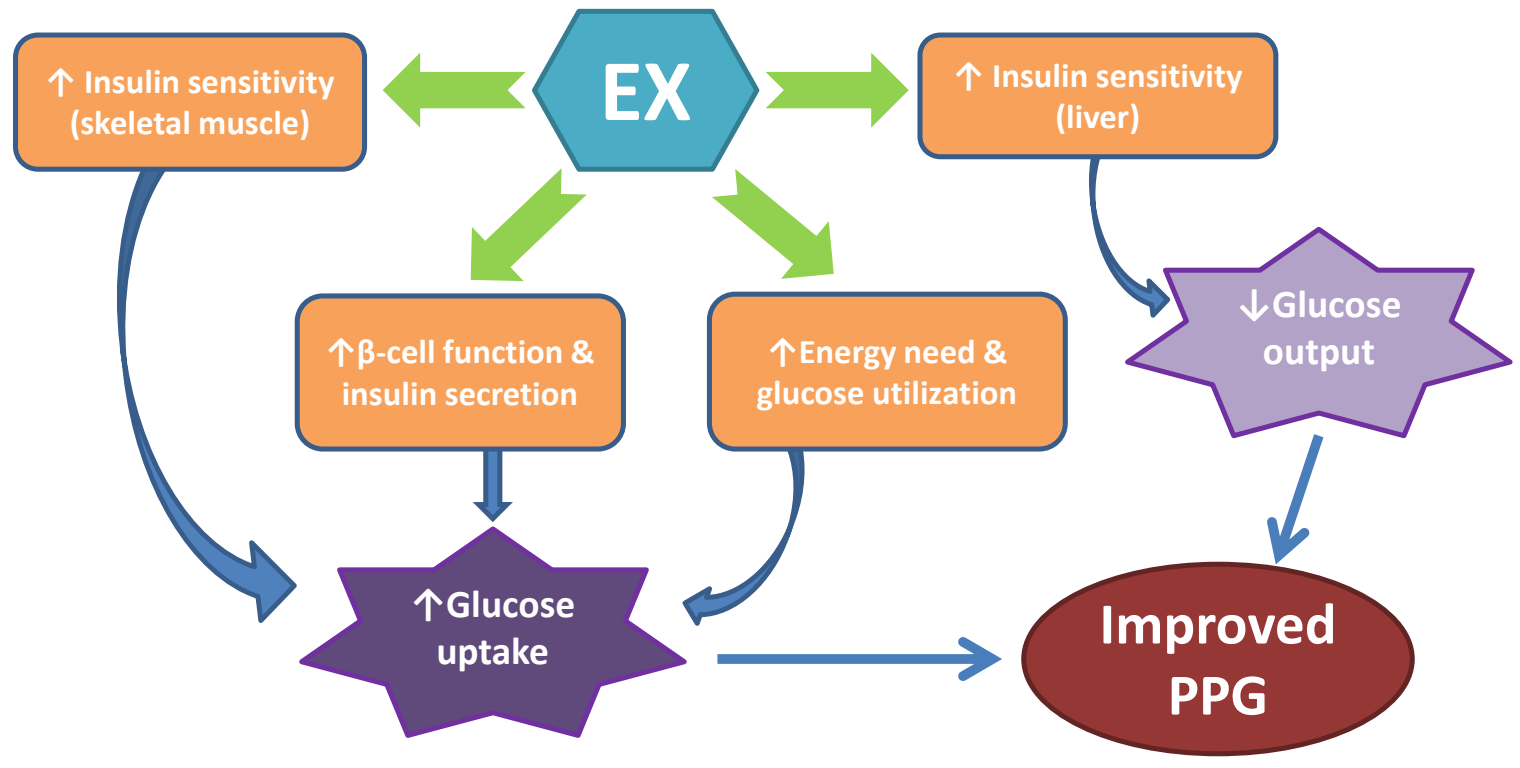

FIGURE 1 - Exercise (EX) improves postprandial glycemia (PPG) through multiple pathways involving increased glucose uptake and decreased glucose output. 
Additionally, $\beta$-cell function and thus insulin secretion improves somewhere along the course of exercise training (148). This may be related to a variety of mechanisms, including reductions in circulating glucose requiring a lesser demand on the $\beta$-cell, and reductions in oxidative stress (135-137). Understanding what cells, tissues, and organs show improvements after a week of exercise training will begin to give us a time line of when and how each of these affects glycemic control with exercise and exercise training.

Before exercise will become accepted as a first-line treatment, many open questions must be answered regarding how, why, and when exercise improves glycemia. First, it is important to define what is meant by the term exercise in this capacity. Exercise has a vast array of modalities and is used in the literature to define anything from single, acute bouts, to short-term training (usually 5-7 days), to longer term training (often 8 weeks to several years).

In most of the literature on exercise and glycemic control, aerobic exercise training of either short-term or long-term duration is typically examined. However, even an acute bout of exercise has been shown to improve some, but not all, measures of glycemic control over a 1-2 day period in subjects with T2D $(103,121)$. Additionally, seven days of exercise improves glycemic control in T2D patients as assessed by a freeliving measure, CGMS, but not by a typical lab challenge, the OGTT $(108,109)$. Longer term exercise training, however, shows enhanced improvements in glycemic control measured by HbA1c, both in the presence and absence of changes in body composition $(22,52,144)$. This suggests that exercise in an acute bout may have different mechanisms for improvement than does exercise training, and longer-term exercise training may also 
be contributing to improved glycemic control differently than short-term exercise training. Importantly, the seven day exercise model is unique in that it provides a window whereby training effects are starting to accumulate and glucose tolerance is improved, but there are not yet changes in many of the traditional markers of training and fitness including maximal oxygen consumption, body weight, or body composition (139).

\section{Assessing glycemic control}

Another important question remains regarding how to most effectively assess glycemic control. Lab-based measures are often non-representative of a human's freeliving condition. The OGTT, in which a $75 \mathrm{~g}$ bolus of glucose is ingested in liquid form, is one such example. While it does not represent what occurs when a person consumes a standard meal outside of the lab, it is the most validated and by far the most used challenge test in scientific and clinical diabetes literature. A more physiological mixedmeal tolerance test (MMTT), in which carbohydrates, protein, and fat are ingested in the form of a meal (in either solid or liquid composition), would be preferential in determining what responses occur in the free-living condition. Although carbohydrates are the primary driver of PPG and the insulin response following meals, protein and fat also have effects on digestion and absorption $(78,79,178,179)$. Notably, the addition of protein and fat to meals decreases the glycemic load of the meal and slows absorption of carbohydrates (79) In addition, dietary lipids have been shown to trigger an incretin response through both gastric inhibitory polypeptide (GIP) and glucagon-like peptide-1 (GLP-1) (104, 115), and protein may stimulate insulin secretion through an increase in ileal mammalian target of rapamycin (mTOR) signaling of GLP-1 (180). With this 
enhanced incretin response, this should result in increased circulating insulin and decreased glucagon to promote better glucose uptake (163). While using a MMTT would mimic the typical meal consumed in vivo, it is difficult to standardize and has not been validated against other measures, including the OGTT.

Little work has been done to assess the validity of a MMTT in assessing postprandial glycemic control. Though one study compared the OGTT with a MMTT (177), examining differences in glucose and insulin concentrations and how subjects tolerated each meal (taste, gastrointestinal distress, nausea, etc.), this study did not examine $\beta$-cell function, measures of insulin sensitivity, or any measures of or relating to glycemic control other than blood glucose and insulin concentrations. Furthermore, this study did not evaluate whether or not each test would differ in its ability to detect changes in glycemic control with an intervention such as exercise training. Finally, the meal used in this study is only one example of a mixed meal and was provided in the form of a dry wafer; no standards currently exist as to what should constitute a mixed meal challenge, a notable weakness of the technique. A few other studies have also attempted to compare the MMTT and the OGTT, but each of these also has weaknesses, and none of them compare changes following an intervention such as exercise $(25,134,165)$.

Another measure of glycemic control gaining popularity is CGMS. This device, which involves a small catheter inserted subcutaneously in the abdomen, measures interstitial glucose concentrations on a continuous basis throughout the day and can be easily used outside of the lab, in a subject's free-living environment. Thus, CGMS allows 24 hour monitoring in normal life and will show changes in glycemia throughout a subject's normal day. The validity of CGMS lies in helping us understand a subject's 
normal responses and how they are altered with exercise. Additionally, when using CGMS in combination with standardized meals and monitored exercise sessions in which timing of meal ingestion and exercise is marked, direct outcomes can be assessed and comparisons may be drawn. CGMS data provide average glycemic control across days and meals, glucose peaks and nadirs, episodes of and time spent in hypoglycemia and hyperglycemia, standard deviation of glycemia, and mean amplitudes of glycemic excursions, all of which give us a view of how glucose fluctuations differ throughout the day and in response to exercise.

\section{Purpose of the current studies}

Collectively, the studies in this dissertation addressed the central hypothesis that short-term aerobic exercise training improves PPG and PPGE through improved muscle insulin sensitivity and glucose uptake and decreased hepatic glucose production, and that testing methods simulating the free-living condition are more meaningful for assessing changes in glycemic control following exercise training.

\section{SPECIFIC AIMS}

In this dissertation, the following specific aims were addressed utilizing a shortterm exercise training intervention with a longitudinal study design in subjects with wellcontrolled T2D. Testing these aims provided answers to the questions of how exercise training improves glycemic control after only one week and what tissues are involved in these outcomes. In addition, it was thought that this study would help to validate the use of the MMTT in the laboratory setting and determine if it is more or less sensitive to 
changes in glycemic control with exercise training when compared to the standard $75 \mathrm{~g}$ OGTT. Collectively, these studies progressed the knowledge of how exercise improves glycemic control and how to more accurately measure these changes, which should ultimately improve clinical outcomes should exercise be provided in a larger therapeutic capacity in patients with T2D.

\section{Specific aim 1}

The primary aim of this dissertation was to identify tissues contributing to changes in glycemic control after short-term exercise training (e.g. muscle, liver, pancreas) and determine what the systemic mechanisms are by which exercise lowers PPGE and improves overall glycemic control in patients with T2D. To study this, we utilized lab-based MMTTs with dual glucose tracers $\left(\left[6,6-{ }^{2} \mathrm{H}_{2}\right]\right.$ glucose and $\left[\mathrm{U}_{-}{ }^{13} \mathrm{C}_{6}\right]$ glucose) combined with measures of pancreatic $\beta$-cell function and muscle insulin sensitivity to assess changes in skeletal muscle glucose disposal, control of endogenous glucose production, and pancreatic $\beta$-cell function, and how these relate to changes in PPG. In addition to the MMTT measures of PPG, subjects also were outfitted with CGMS during the tests to compare glycemic control in the free-living condition with labbased measures.

Hypothesis for aim 1. It was hypothesized that short-term exercise training would improve glucose disposal and decrease hepatic glucose output in patients with T2D. However, we did not think one week would be enough of a training stimulus to result in significant improvements in pancreatic $\beta$-cell function in this population (Figure 2). 


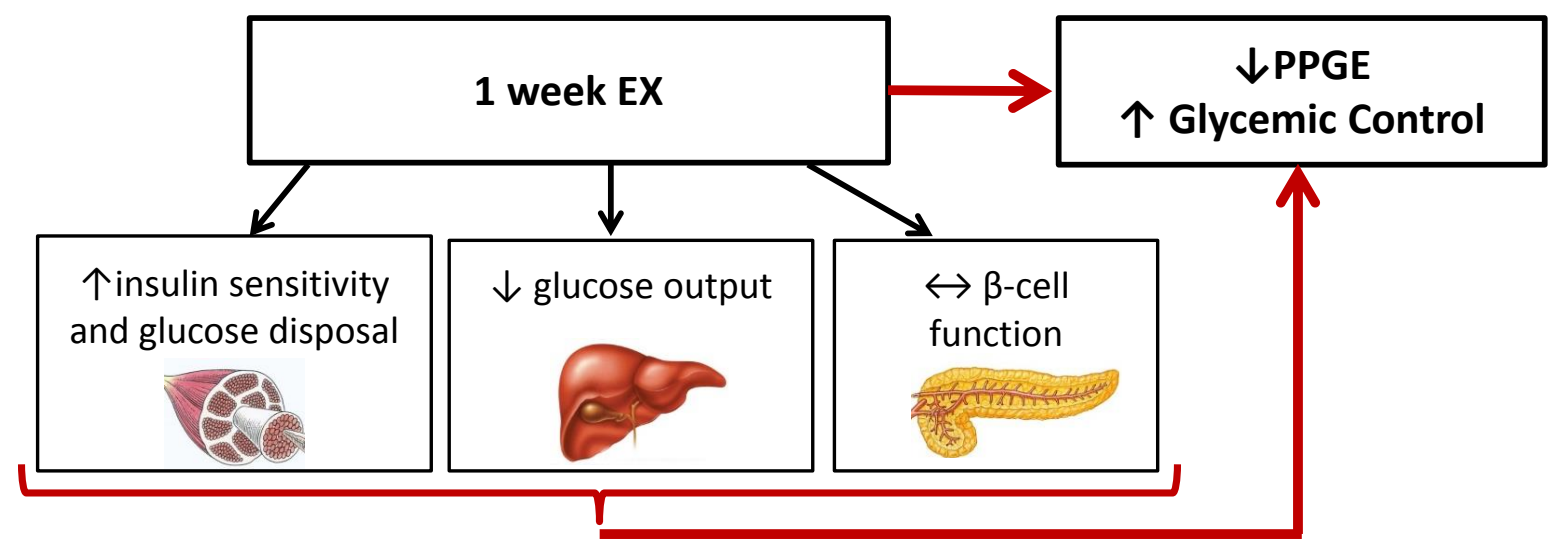

FIGURE 2 - Schematic representation of the hypothesis for aim 1. One week of aerobic exercise training (EX) will lower postprandial glycemic excursions (PPGE) and improve glycemic control through improved skeletal muscle insulin sensitivity and glucose disposal and decreased endogenous glucose production, or hepatic glucose output. However, the short-term training stimulus will not significantly affect $\beta$-cell function. 


\section{Specific Aim 2}

The secondary aim of this dissertation was to determine, in patients with $\mathrm{T} 2 \mathrm{D}$, if a MMTT is a more effective tool for assessing improvements in glycemic control following exercise training than the standard OGTT. Subjects in this study underwent both labbased MMTT and OGTT prior to and following one week of exercise training to determine which method provides a more sensitive determination of changes in postprandial glycemic control following a short-term exercise training intervention.

Hypothesis for aim 2. It was hypothesized that measures of postprandial glycemic control from the MMTT would be significantly improved following exercise training while the OGTT would not show improvements in glycemic control. 


\section{STUDY ONE}

\section{SHORT-TERM AEROBIC EXERCISE TRAINING IN TYPE 2 DIABETES: EVALUATION OF GLYCEMIC CONTROL}

\section{Introduction}

Type 2 diabetes (T2D) is an epidemic both in the United States (U.S.) and throughout the world $(15,33)$. In the U.S. alone, diabetes presents in over 29 million people, or $9.3 \%$ of the nation's population, and $>90 \%$ of all diabetes cases are type 2 (15). Further, T2D results in reduced quality and years of life for those directly afflicted, and it also encumbers society with a large financial burden through drastically increased health care expenditures $(15,22)$.

Poor glycemic control is central to the development and dysfunction of T2D (63), and people with T2D have both elevated fasting hyperglycemia and impaired glucose control after feeding (postprandial glycemia; PPG), as well as chronically high blood glucose as measured by hemoglobin A1c (HbA1c) $(2,6,63)$. While post-absorptive and chronic glycemia are undoubtedly important, as evidenced by large scale trials assessing endpoints associated with $\mathrm{HbA1c}(54,59)$, impaired PPG by itself also increases risk for cardiovascular morbidity and mortality in people with T2D $(5,13)$. Additionally, overall PPG as well as extreme shifts or excursions in glucose after a meal, sometimes called postprandial glycemic excursions (PPGE), appear to be more detrimental to health than average glucose concentrations as assessed by $\operatorname{HbA1c}(14,16,17,29,61)$. Further, it is important to study changes in PPG in response to interventions targeted to improve 
glycemic control, as they may or may not be related to overall blood glucose concentrations throughout the day or averaged over time.

Since T2D is such a large scale problem, effective therapies are needed which will improve PPG. Yet, current pharmaceutical options are aimed at reducing HbA1c, a chronic measure of average glucose concentration, rather than PPG. Exercise, on the other hand, is an effective treatment for improving PPG $(41,46)$, in addition to reducing overall hyperglycemia as assessed by $\operatorname{HbA1c}(4,53)$. Aerobic exercise improves both insulin and non-insulin stimulated glucose uptake $(26,30,40)$, which is thought to be its primary mechanism of enhanced glycemic control. Exercise training also impacts other tissues related to glycemic control, including the liver and the pancreatic $\beta$-cell (43), both of which are influential in reducing PPG. While much has been discovered about the benefits of exercise in $\mathrm{T} 2 \mathrm{D}$, the precise time course of exercise training changes occurring in these tissues in patients with T2D is still unknown.

The one week model of exercise provides for a unique view of exercise training because it allows us to determine what the addition of daily exercise does independent of chronic training adaptations known to impact insulin sensitivity and glycemic control including improved body composition, increased maximal oxygen consumption $\left(\mathrm{VO}_{2 \max }\right)$, or glucose transporter 4 (GLUT4) protein content (51). One week of exercise training ( 1 hour per day) has been previously shown to improve insulin-stimulated glucose uptake, PPG, and PPGE (40, 45, 46, 51), but the mechanisms of these changes have yet to be elucidated. Additionally, most studies assessing PPG do not use a true meal challenge but instead examine glucose responses to pure glucose ingestion (via an oral glucose tolerance test; OGTT). However, the highly concentrated glucose beverages 
used in the OGTT do not accurately represent the physiological condition because they do not match the mixed macronutrients (containing carbohydrate, proteins, and fats) that are commonly ingested by humans on a day to day basis.

Therefore, the purpose of the present study was to identify, in patients with T2D, what tissues (muscle, liver, and pancreas) contribute to changes in glucose control after consumption of mixed meals following a short-term, one week, exercise training intervention. We utilized a lab-based mixed meal tolerance test (MMTT) with dual

glucose tracers $\left(\left[6,6-{ }^{2} \mathrm{H}_{2}\right]\right.$ and $\left[\mathrm{U}_{-}{ }^{13} \mathrm{C}_{6}\right]$ glucose) combined with measures of pancreatic $\beta$-cell function and muscle insulin sensitivity to assess changes in skeletal muscle glucose disposal, control of endogenous glucose production, and pancreatic $\beta$-cell function, and how these relate to improvements in PPG, PPGE, and 24 hour glycemic control. It was hypothesized that one week of exercise training would improve glucose disposal and decrease hepatic glucose output in patients with T2D but that pancreatic $\beta$-cell function would not be improved after only one week of training.

\section{Methods}

The study protocol was approved by the Health Sciences Institutional Review Board at the University of Missouri. All subjects provided written informed consent before participation.

\section{Subjects}

Sedentary and low-activity (0-1 sessions/week of physical activity lasting 30 minutes or more and not employed in a physically active job or hobby) overweight to obese [body mass index (BMI) $25.0-37.0 \mathrm{~kg} / \mathrm{m}^{2}$ ] male and female subjects with 
physician-diagnosed T2D were recruited for participation. Subjects were older (48-67 years), non-smokers, not on exogenous insulin therapy, had T2D with an $\mathrm{HbA} 1 \mathrm{c}<10 \%$, and reported no previous cardiac events. Additionally, all subjects were screened for comorbidities using a combination of questionnaires, a fasting blood sample, and a physician-monitored maximal exercise stress test with electrocardiography (ECG). They were weight-stable ( $<5 \%$ weight change in previous 3 months), were not breakfast skippers and had no other reported irregular dietary patterns, and had no physical limitations which would restrict them from engaging in a structured exercise program. Nine subjects took at least one medication for diabetes management (biguanide: $\mathrm{n}=9$, sulfonylurea: $\mathrm{n}=2$, GLP-1 receptor agonist: $\mathrm{n}=1$ ), and all but one subject also took medications for either hypertension or dyslipidemia, or both ( $\beta$-blocker: $n=3, A C E$ inhibitor: $n=4$, diuretic: $n=6$, angiotensin II receptor antagonist: $n=2$, statin: $n=6$, fibrate: $n=2)$.

Subsequent to an initial phone screen and provision of written informed consent, subjects completed a medical history questionnaire, a dietary questionnaire, and a food and activity record before returning to the exercise physiology lab. Following a 12 hour overnight fast, subjects returned to the lab for a fasting blood draw used to assess blood glucose, HbA1c, and blood lipids [total cholesterol, low-density lipoprotein cholesterol (LDL-C), high-density lipoprotein cholesterol (HDL-C), and triglycerides (TAG)] by a commercial laboratory, as done previously (46). Additionally, subjects underwent anthropometric assessment for height and weight during this visit and a dual energy $\mathrm{x}$-ray absorptiometry (DXA) scan (Hologic QDR 4500A; Hologic, Bedford, MA) for determination of body composition. 
On a subsequent visit, subjects reported to the Clinical Research Center (CRC) at the University of Missouri for participation in a maximal exercise stress test for determination of peak oxygen consumption $\left(\mathrm{VO}_{2 \text { peak }}\right)$. The physician-monitored test was done on a treadmill using the Bruce Protocol in accordance with guidelines outlined by the American College of Sports Medicine (ACSM), as performed previously $(1,48)$. Heart rate, blood pressure, and respiratory gases were monitored throughout the test, and subjects were encouraged to continue to exhaustion or until they met three of the criteria for achieving a maximal exercise test as described by ACSM (1). Respiratory gases were measured and oxygen consumption $\left(\mathrm{VO}_{2}\right)$ assessed using a metabolic cart (ParvoMedics True One 2400 Metabolic Measurement System; ParvoMedics, Sandy, UT), and cardiovascular function was monitored using a 12-lead ECG (Quinton Q-Stress v3.5 Exercise Test Monitor; Quinton Cardiology, Inc./Burdick, Deerfield, WI). A cardiologist reviewed ECG data for each exercise test to ensure that subjects could safely participate in an exercise training program. Following the exercise stress test, there was a minimum one-week washout period before subjects began the study protocol to eliminate any residual effects of the testing exercise bout.

\section{Experimental design}

Metabolic parameters relating to postprandial glycemic control were compared before (Pre) and after (Post) a seven day exercise intervention in subjects with T2D using a continuous glucose monitoring system (CGMS) and mixed meal tolerance test (MMTT) with stable isotope glucose tracers. This pre-post longitudinal design allowed for direct comparisons of changes within subjects. The study design is outlined in Figure 3. 


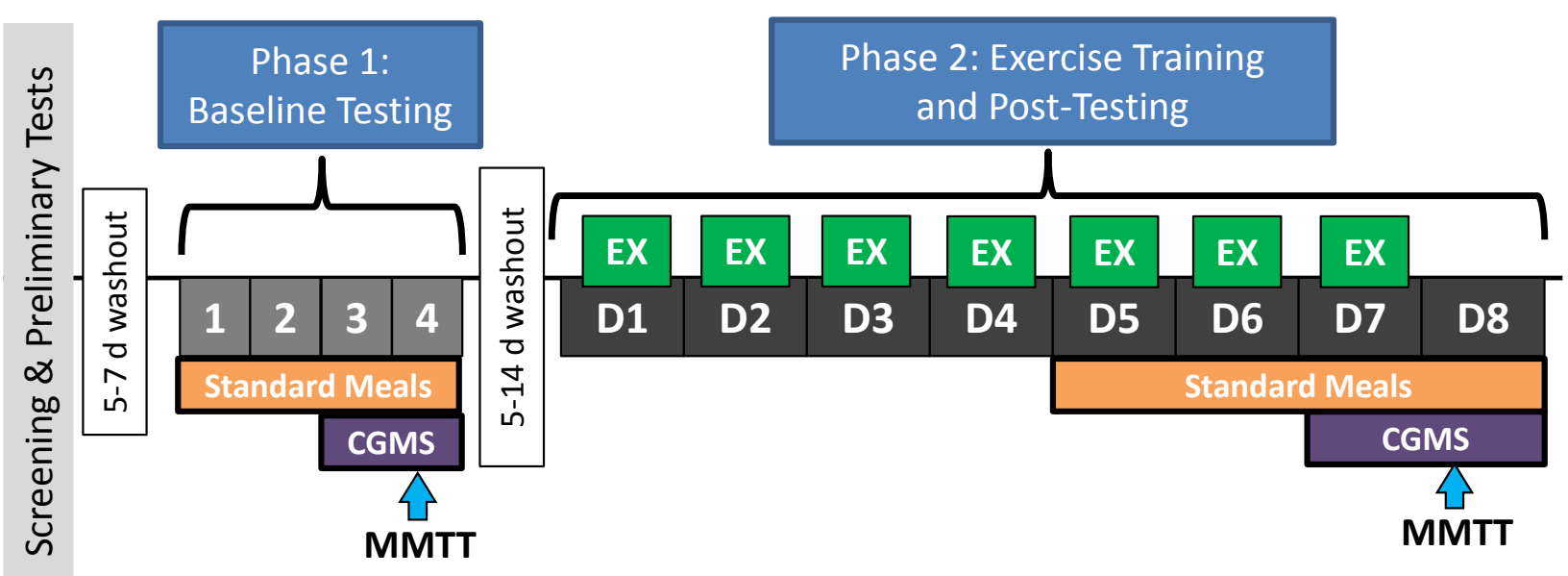

FIGURE 3 - Study design and time line (EX=60 minutes of aerobic exercise

training; $\mathrm{D}=$ day of exercise training and post-testing period; $C G M S=c o n t i n u o u s$

glucose monitoring system; MMTT=mixed meal tolerance test). 


\section{Exercise prescription and training}

Following a graded exercise test to determine fitness and maximal heart rate, aerobic exercise was prescribed at $60 \%$ of heart rate reserve (HRR). This intensity has been shown previously to relate to maximal oxygen consumption $\left(\mathrm{VO}_{2 \max }\right)$ and correlate with reserve oxygen consumption $\left(\mathrm{VO}_{2 \text { reserve }}\right)(9,60)$ and was chosen because it is considered to be at the high range of moderate intensity $(9,27)$ and can be tolerated in this population, as evidenced by previous studies $(45,46,48)$. Additionally, this exercise duration and intensity is in agreement with recommendations by the ACSM and the American Diabetes Association for patients with T2D $(18,19)$. HRR was calculated as described by Karvonen and Vuorimaa (39) using the peak heart rate ( $\left.\mathrm{HR}_{\text {peak }}\right)$ and resting heart rate $\left(\mathrm{HR}_{\text {rest }}\right)$ values attained during the exercise stress test, with the following formula: $\mathrm{HRR}=\left[\left(\mathrm{HR}_{\text {peak }}-\mathrm{HR}_{\text {rest }}\right) \times \%\right.$ intensity $]+\mathrm{HR}_{\text {rest }}$.

Daily exercise training consisted of 20 minutes of brisk treadmill walking followed immediately by 20 minutes of stationary cycling and then another 20 minutes of treadmill walking, totaling 60 minutes of exercise per session. All exercise sessions were supervised in the Exercise Physiology fitness laboratory by trained study personnel. Seven consecutive days of exercise training were performed prior to the MMTT. Subjects performed the majority of exercise sessions in the late afternoon, before the dinner meal, and the last exercise session before the MMTT was always completed 14-16 hours prior to the MMTT study day testing meal. Exercise intensity was monitored continuously throughout each exercise session using heart rate telemetry. To minimize subject discomfort and reduce risk for injury and boredom, subjects alternated from the treadmill to the bike and back to the treadmill for the workout, as this model has previously shown 
to be successful in diabetic populations with low fitness (46). Blood glucose was taken via a finger prick using a handheld glucometer before and after each exercise session, and subjects' feet were checked for sores, blisters, or other abrasions prior to each workout as well as following the exercise sessions. If blood glucose values were $<100 \mathrm{mg} / \mathrm{dL}$ prior to exercise, a snack was administered and glucose concentration was re-checked before initiation of exercise. To offset the energy expenditure from the exercise sessions, snacks of 350 kilocalories (kcal) were given to compensate for the energy deficit and prevent weight loss, as we have done previously (46).

\section{MMTT experimental protocol}

A MMTT with stable isotope glucose tracers, $\left[\mathrm{U}^{13} \mathrm{C}_{6}\right]$ glucose $(13 \mathrm{C}$; administered orally) and $\left[6,6-{ }^{2} \mathrm{H}_{2}\right]$ glucose (d2; infused) (Cambridge Isotope Laboratories, Inc., Tewksbury, MA) was performed before (Pre) and after (Post) one week of exercise training. Each MMTT was performed on the morning of the second CGMS monitoring day, 14-16 hours after the last exercise session and following a 12 hour fast, at the CRCat the University of Missouri School of Medicine. Subjects were asked to refrain from taking their morning medications on the day of the test, for both conditions, to eliminate any acute pharmaceutical influences. The meal for the MMTT was exactly the same as what was provided for the breakfasts for all CGMS testing days (breakfast wrap with orange juice) except that the MMTT in the laboratory had $\left[\mathrm{U}_{-}{ }^{13} \mathrm{C}_{6}\right]$ glucose put into the orange juice, while $\left[6,6-{ }^{2} \mathrm{H}_{2}\right]$ glucose was infused intravenously.

The testing design for each MMTT is depicted in Figure 4. Subjects were admitted to the CRC between 6:30 and 7:00 A.M. following a 12 hour overnight fast. A first-void urine sample was provided, and subjects were weighed before reclining in a bed 
for testing preparation. Inline catheters were placed by a research nurse into an antecubital arm vein in one arm for infusion of $\left[6,6-{ }^{2} \mathrm{H}_{2}\right]$ glucose and the antecubital arm vein in the other arm for repeated blood sampling. The arm used for blood sampling was immediately placed in a heated box to provide for sampling of arterialized venous blood, as described previously (57). A baseline blood sample was taken, and an intravenous priming infusion of $\left[6,6-{ }^{2} \mathrm{H}_{2}\right]$ glucose $[20 \mu \mathrm{mol} / \mathrm{kg}$ bolus (adjusted based on fasting plasma glucose)] was administered. Immediately following the priming bolus infusion, a continuous infusion $(0.2 \mu \mathrm{mol} / \mathrm{kg} / \mathrm{min})$ was started, and the clock was started and time recorded as -120 (120 minutes prior to consumption of the mixed meal). This infusion was continued throughout the duration of the test, to 240 minutes following meal consumption. 


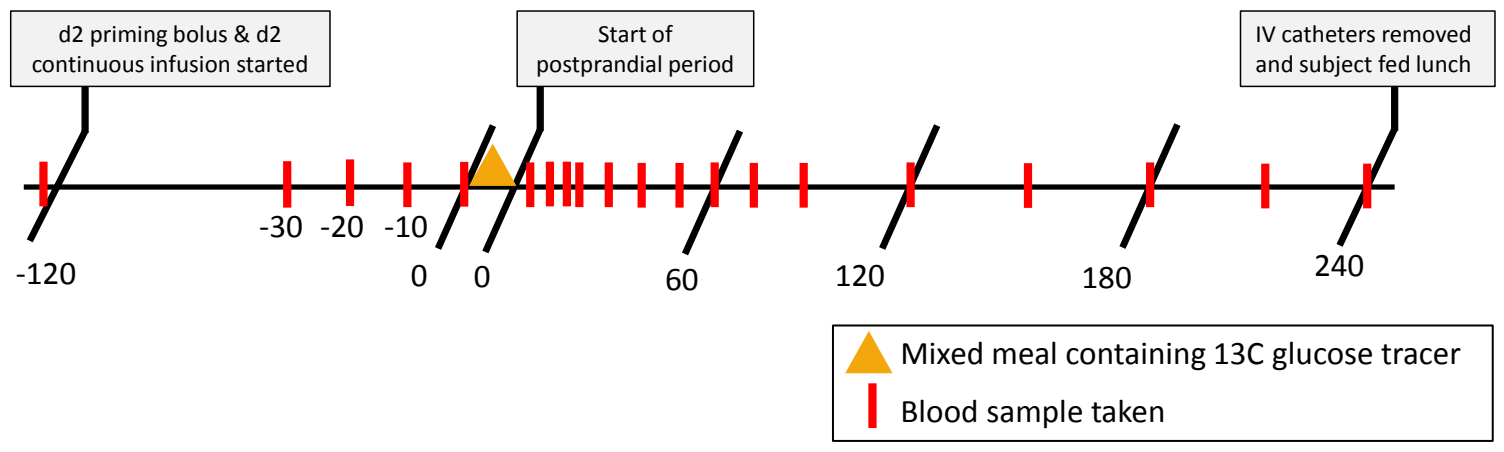

FIGURE 4 - Experimental protocol for the mixed meal tolerance test (MMTT). 
Following a blood sample at time zero, subjects consumed a 400 kilocalorie (kcal) mixed meal (Table 1; breakfast wrap and orange juice; 60\% carbohydrate, $30 \%$ fat, $10 \%$ protein). The meal composition was chosen to mirror the typical caloric content of breakfasts consumed in the United States (38). The orange juice in the meal contained $\left[\mathrm{U}-{ }^{13} \mathrm{C}_{6}\right]$ glucose at $>5 \%$ enrichment. The meal was consumed in approximately five minutes, with the exact consumption time for each subject recorded in the Pre condition and replicated in the Post condition. Half of the orange juice was sipped throughout the five minutes while the subject ate the breakfast wrap and the other half was consumed immediately following the last bite of the wrap. This was done to both promote adequate mixing of the tracer with the meal in the stomach and to ensure that half of the tracer was consumed at time zero for each subject. Following consumption of the orange juice, the drinking glass was rinsed twice with $10 \mathrm{~mL}$ of water, which the subject consumed to ensure that all of the $\left[\mathrm{U}_{-}{ }^{13} \mathrm{C}_{6}\right]$ glucose was ingested. The clock was immediately restarted to time zero once all fluid was ingested. Blood was sampled from the arterialized venous site at $-120,-30,-20,-10,0,5,10,15,20,30,40,50,60,75,90,120,150,180,210$, and 240 minutes. Samples were collected in serum separator (SST) and EDTA tubes with aprotinin (a protease inhibitor) and dipeptidyl peptidase IV (DPP-IV) inhibitors added. Collection tubes were centrifuged at $3500 \mathrm{rpm}$ for 15 minutes, at $4^{\circ} \mathrm{C}$, and plasma and serum were aliquoted and stored at $-80^{\circ} \mathrm{C}$ until time of analysis. 
TABLE 1. Composition of the breakfast meal for MMTT testing and for the study diet.

\begin{tabular}{lrrrrr}
\hline Food & $\begin{array}{r}\text { Serving } \\
(\mathbf{g})\end{array}$ & $\begin{array}{r}\text { CHO } \\
(\mathbf{g})\end{array}$ & $\begin{array}{r}\text { Fat } \\
(\mathbf{g})\end{array}$ & $\begin{array}{r}\text { Protein } \\
(\mathbf{g})\end{array}$ & $\begin{array}{r}\text { Energy } \\
(\mathbf{k c a l})\end{array}$ \\
\hline Flour tortilla & 45.9 & 23.4 & 3.3 & 3.7 & 140.5 \\
Egg beaters & 2.0 & 0 & 0 & 0.2 & 1.1 \\
Egg yolk (from large egg) & 7.7 & 0.2 & 1.2 & 0.7 & 14.5 \\
American cheese & 18.0 & 1.7 & 4.3 & 2.6 & 60 \\
Margarine spread & 9.0 & 0 & 4.5 & 0 & 38.6 \\
100\% orange juice, pulp free & 321.0 & 34.8 & 0 & 2.7 & 147.1 \\
TOTAL & & $\mathbf{6 0 . 1}$ & $\mathbf{1 3 . 3}$ & $\mathbf{9 . 9}$ & $\mathbf{4 0 1 . 8}$ \\
\hline
\end{tabular}




\section{Continuous glucose monitoring}

After recruitment and screening, subjects were outfitted with a continuous glucose monitoring system (CGMS; iPro2, Medtronic Diabetes, Minneapolis MN) which was worn for two days (the day before the MMTT and during the MMTT study day) for the Pre condition and then repeated for two days at the end of the 7 days of exercise training (Post condition). Glucose sensors were placed subcutaneously in the periumbilical region and connected to the CGMS monitor either the night before or early in the morning the day prior to the MMTT, a minimum of one hour before the subject consumed breakfast. Subjects were instructed to take four finger stick blood glucose measures per day during the CGMS periods and record precisely when all meals were consumed, as done previously $(45,46)$. Additionally, they were asked to leave at least three hours between each meal and snack so that postprandial glucose could be assessed before the next meal occurred. The meals (both food provided and caloric content, as well as meal-timing) during CGMS monitoring periods were exactly the same during both Pre and Post exercise training, with the one exception of post-exercise snacks consumed in the Post condition.

To control for potential variation in dietary intake during CGMS, subjects consumed a controlled study diet for two days prior to and during the CGMS monitoring period. The meals consisted of common palatable foods and were prepared as pack-out meals by the metabolic kitchen at the University of Missouri. Dietary composition was $60 \%$ carbohydrate, $30 \%$ fat, and $10 \%$ protein, provided as three main meals and an afternoon snack. The total caloric content was prescribed according to each subject's individual energy needs to maintain energy balance calculated by the Harris-Benedict 
equation, rounded to the nearest $200 \mathrm{kcal}$ (31). For the first three subjects, the result of the Harris-Benedict equation was averaged with the subject's mean reported three day dietary caloric intake; because this did not change the prescribed study diet compared to using the Harris-Benedict equation alone, the Harris-Benedict equation was used in solitude for the remaining subjects. With the exception of additional snacks (350 kcal) consumed in the exercise condition to offset energy expenditure, as done previously (45, 46), all meals were exactly the same each day and for each condition.

CGMS values were determined by Carelink Minimed iPro2 software specific to the CGMS model used (Medtronic CareLink iProTherapy Management Software for Diabetes). CGMS data collected at baseline and after one week of exercise were used to calculate measures of glycemic control, PPG, and PPGE, including 24 hour average glucose concentrations, number of hyperglycemic (>10 mM glucose) and hypoglycemic ( $<4 \mathrm{mM}$ glucose) episodes, average incremental area under the curve (iAUC) for glucose following meals, daily maximum and minimum glucose levels, and differences between maximum and minimum glucose following meals, as has been performed previously (46, 48).

\section{Blood analyses and calculations for the MMTT and OGTT}

Serum samples collected from the MMTTs were analyzed for glucose by the glucose oxidase method (Sigma, St. Louis, MO), as done previously (47). Insulin and cpeptide were analyzed by enzyme-linked immunosorbent assay (IMMULITE 1000 Analyzer, Siemens, Inc., Deerfield, IL).

$\boldsymbol{A} \boldsymbol{U C}$ and $\boldsymbol{i} \boldsymbol{A} \boldsymbol{U C}$. Paired sample t-tests were used to assess baseline differences for glucose, insulin, and c-peptide for determination of whether total area under the curve 
(AUC) or iAUC should be used in analyses. Because no baseline differences were found for insulin or c-peptide, total AUC was calculated for each of these measures, while iAUC was used for glucose because of small but statistically significant differences between glucose concentrations at baseline. Glucose iAUC and AUC for insulin and cpeptide were calculated by the trapezoidal method, with the former adjusted with respect to increase from baseline, as described previously $(12,50,64)$.

Percent (\%) change. The \% change in variables from Pre to Post was calculated using the following formula: $\%$ change $=[($ Post-Pre $) /$ Post $] * 100$. Negative $\%$ change values indicated a reduction in the variable with exercise training, while positive $\%$ change indicated that the variable increased with exercise training.

Insulin sensitivity. The calculated Matsuda insulin sensitivity index (ISI) was used as an estimate of insulin sensitivity and was calculated using baseline and average glucose and insulin concentrations throughout the tests as described previously (44). A second determination of a change in insulin sensitivity with this Pre/Post design was a comparison between changes in glucose iAUC and insulin AUC, as has been defined by the relationship between insulin resistance and hyperinsulinemia (52). Briefly, a reduction in the postprandial insulin response with no change in glucose concentrations is indicative of improved insulin sensitivity, while an increase in postprandial insulin concentrations with no change or a decrease in glucose concentrations indicates reduced insulin sensitivity (52).

$\boldsymbol{\beta}$-cell function. The ratio of the change in c-peptide concentrations to the change in glucose concentrations early in the test was used to estimate $\beta$-cell function using the formula: $\beta$-cell function $=\Delta$ c-peptide $(30-0) / \Delta$ glucose $_{(30-0)}$, as done by others $(55)$. 
Oral disposition index $\left(\boldsymbol{D I}_{O}\right)_{\text {. }}$. The disposition index for examination of insulin sensitivity and $\beta$-cell function in response to an ingested challenge $\left(\mathrm{DI}_{\mathrm{O}}\right)$ was calculated as the product of the insulinogenic index and the reciprocal of fasting insulin concentration: $\mathrm{DI}_{O}=\left[\Delta \operatorname{insulin}_{(0-30)} / \Delta\right.$ glucose $\left._{(0-30)}\right] \times$ (1/fasting insulin), as described previously (62). The $\mathrm{DI}_{\mathrm{O}}$ is a version of the disposition index modified by Kahn's group from the traditional disposition index (36) to be used for studies where glucose is ingested orally rather than infused intravenously.

Glucose isotope analysis and kinetic modeling. Plasma $\left[6,6-{ }^{2} \mathrm{H}_{2}\right]$ glucose and [U${ }^{13} \mathrm{C}_{6}$ ] glucose enrichment were measured by gas-chromatography mass spectroscopy (GC-MS) at Washington University as performed previously (11) and used to calculate the tracer to tracee ratio (TTR) for each isotope. Calculations of total glucose rate of appearance $\left(\mathrm{Ra}_{\text {total }}\right)$, oral glucose rate of appearance $\left(\mathrm{Ra}_{\text {oral }}\right)$, endogenous glucose production (EGP), and glucose rate or disappearance (Rd) (glucose disposal) were performed as previously described $(11,28)$.

Glucose $\mathrm{Ra}_{\text {total }}$, or the rate of appearance of all glucose into the bloodstream (both from glucose ingested in the meal and production from endogenous sources), was calculated based on Steele's equation for single-pool, non-steady state kinetics (58) as described by Gastaldelli et al. (28), normalized to total body mass (kg). For each time point $(t), \mathrm{Ra}_{\text {total }}$ was calculated as:

$R a_{\text {total }}(t)=\left[I R-C(t) x V x\left(d T T R_{[6,6-2 H 2] ~ g l u c o s e}(t) / d t\right)\right] / T T R_{[6,6-2 H 2] ~ g l u c o s e}(t)$, where IR is the infusion rate of $\left[6,6-{ }^{2} \mathrm{H}_{2}\right]$ glucose, $\mathrm{C}$ is the concentration of plasma glucose, $V$ is a constant value for the volume of distribution $(162.5 \mathrm{ml} / \mathrm{kg})$, $d$ is a smoothed derivative function, and $(t)$ is the value at a particular time point. 
Calculations for glucose $\mathrm{Ra}_{\text {oral }}$ (glucose appearance from the ingested meal), EGP (glucose appearing in the blood from endogenous sources), and glucose $\mathrm{Rd}$ (rate of glucose disposal or uptake; glucose removal from the blood) were also initially performed using previously described formulas (28) but are not reported due to unforeseen complications in modeling starch within the consumed meal, as starch is digested and absorbed more slowly than glucose and sucrose (8). In place of direct comparisons of these outcomes, AUC for the orally ingested $\left[\mathrm{U}_{-}{ }^{13} \mathrm{C}_{6}\right]$ glucose TTR and the for the infused $\left[6,6-{ }^{2} \mathrm{H}_{2}\right]$ glucose TTR were calculated and analyzed independently using paired-sample t-tests to determine if any differences existed in the Pre vs. Post conditions. If Pre vs. Post differences existed, conclusions could not be drawn regarding $\mathrm{Ra}_{\text {oral }}$, EGP, and Rd. However, if there were no differences in either TTR Pre and Post, it was assumed that there were no differences in the aforementioned outcomes since the TTRs were the only values influencing the changeable outcomes of the equations used to calculate $\mathrm{Ra}_{\text {oral }}, \mathrm{EGP}$, and glucose $\mathrm{Rd}$ in this study (28).

\section{Statistical analysis}

Statistical analyses were performed using IBM SPSS Statistical Software, version 22 (IBM Corporation, Armonk, NY). Paired samples t-test were run on time zero measures of glucose, insulin, and c-peptide concentration for determination of whether total AUC or iAUC would be appropriate calculations for comparison of the postprandial responses for these variables.

Paired samples t-tests with Bonferroni correction (7) were used for all determinations in which one outcome variable was assessed for the Pre and Post conditions. Thus, paired samples t-tests were utilized to compare all CGMS outcomes, all 
MMTT outcomes of glycemic control or insulin responses (glucose, insulin, and cpeptide AUC or iAUC), and subsequent MMTT outcomes related to tissue contributions and mechanisms of glycemic control (Matsuda ISI, $\beta$-cell function, $\mathrm{DI}_{\mathrm{O}}$, and $\mathrm{Ra}_{\mathrm{total}}$ ). Statistical significance was set at $\mathrm{p}<0.05$, and all data are expressed as mean \pm standard error of the mean (SEM).

Paired samples t-tests also were used to compare Pre vs. Post differences in AUC for each TTR to confirm that there were no changes in isotope enrichment between conditions.

\section{Results}

Ten subjects ( 7 women, 3 men) completed the study protocol and were included in all MMTT analyses except tracer kinetic modeling. One subject was excluded from tracer modeling analyses due to equipment malfunction during the test that confounded the accuracy of modeling; thus, nine subjects are included in tracer kinetic data outcomes. Baseline subject characteristics are described in Table 2. 
Table 2. Baseline subject characteristics $(n=10)$.

\begin{tabular}{lr}
\hline Variable & $58 \pm 2$ \\
\hline Age (years) & $7 / 3$ \\
Sex (F/M) & $95.0 \pm 6.2$ \\
Body Weight $(\mathrm{kg})$ & $33.9 \pm 1.7$ \\
Body Mass Index $\left(\mathrm{kg} / \mathrm{m}^{2}\right)$ & $38.0 \pm 1.9$ \\
Body Fat $(\%)$ & $159 \pm 8$ \\
Total Cholesterol $(\mathrm{mg} / \mathrm{dl})$ & $85 \pm 7$ \\
LDL-C (mg/dl) & $45 \pm 3$ \\
HDL-C (mg/dl) & $151 \pm 23$ \\
Triglycerides (mg/dl) & $6.8 \pm 0.1$ \\
Hemoglobin Alc $(\%)$ & $128 \pm 10$ \\
Fasting blood glucose $(\mathrm{mg} / \mathrm{dl})$ & $3 \pm 1$ \\
Duration of diabetes $(\mathrm{years})$ & $20.6 \pm 1.1$ \\
Relative VO 2 peak $\left(\mathrm{ml} \cdot \mathrm{kg}^{-1} \cdot \mathrm{min}^{-1}\right)$ & \\
\hline Data are expressed as mean $\pm \mathrm{SEM}$. &
\end{tabular}


Exercise adherence was $100 \%$, with all subjects completing seven consecutive days of supervised exercise training at the appropriate intensity. Further, the re-feeding strategy was effective in maintaining energy balance, as evidenced by maintenance of body weight $(\mathrm{p}>0.05)$.

\section{CGMS}

Due to malfunctions in the recording of the glucose monitors over various time periods, complete data sets were not available for all subjects. Thus, only 7-9 subjects could be used for each of the CGMS determinations. Based on the available data, no statistically significant differences were observed in any of the CGMS measures of 24 hour glycemic control, PPG, or PPGE.

\section{MMTT}

Glucose, insulin, and c-peptide. Because glucose at time 0 was modestly different (determined by a paired samples t-test; $108 \pm 7 \mathrm{mg} / \mathrm{dL}$ Pre vs. $103 \pm 7 \mathrm{mg} / \mathrm{dL}$ Post; $\mathrm{p}<0.05)$, iAUC was calculated for determination of the postprandial glucose response to account for baseline differences. Since there were no differences in insulin at time 0 or c-peptide at time 0 ; thus, total AUC calculations were compared for these measures. While glucose iAUC was not different between conditions, insulin AUC was $20 \%$ lower following exercise training $(11920 \pm 952 \mu \mathrm{IU} \cdot \mathrm{min} / \mathrm{mL} . P r e$ vs. $9979 \pm 645$ $\mu \mathrm{IU} \cdot \mathrm{min} / \mathrm{mL}$ Post; $\mathrm{p}<0.05$ ), and c-peptide AUC was also significantly lower, with a $14 \%$ difference $(1996 \pm 472 \mathrm{ng} \cdot \mathrm{min} / \mathrm{mL}$ Pre vs. $1753 \pm 318 \mathrm{ng} \cdot \mathrm{min} / \mathrm{mL}$ Post; $\mathrm{p}<0.05)$

\section{(Figure 5).}



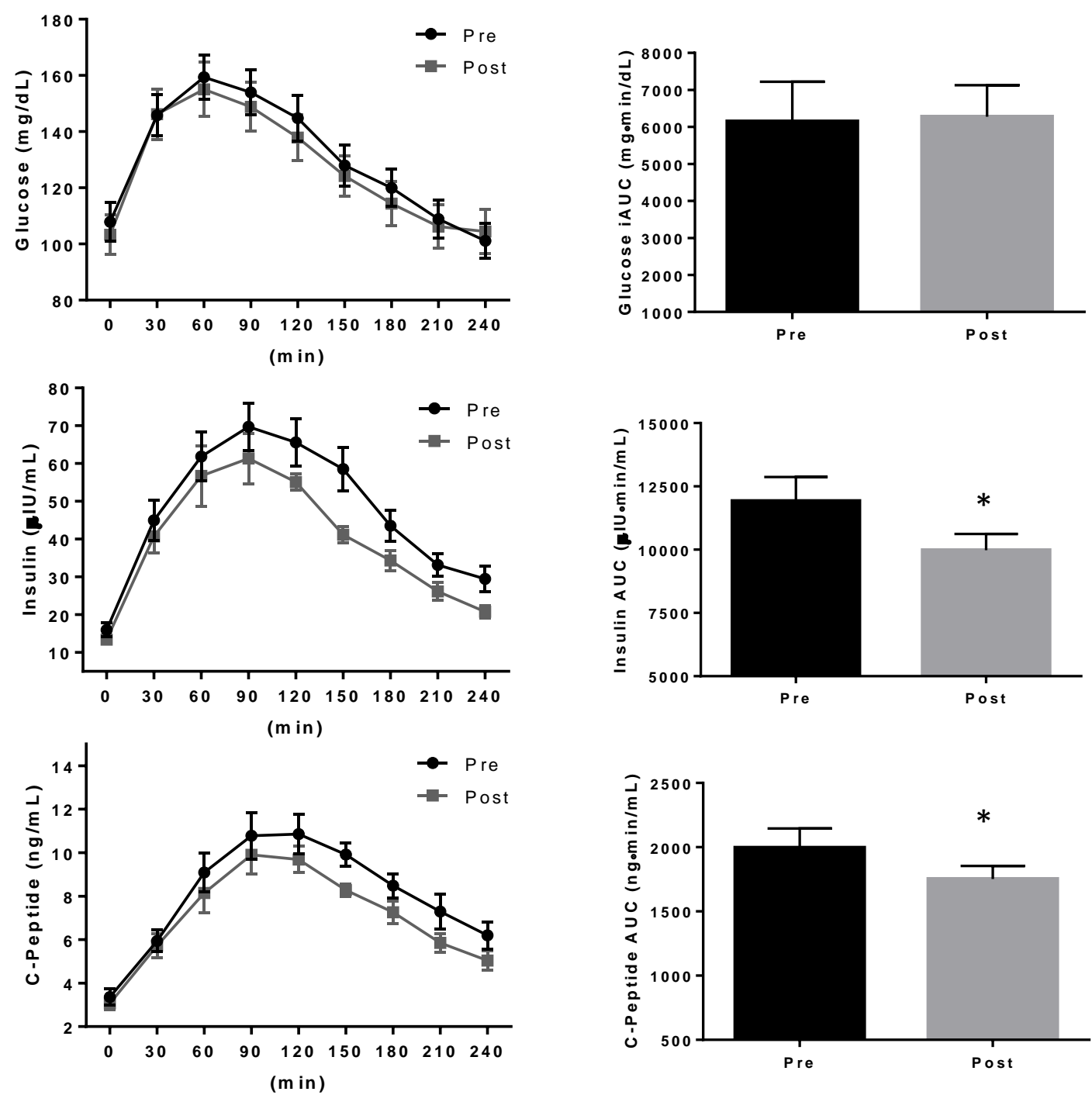

FIGURE 5 - Glucose, insulin, and c-peptide responses to a mixed meal tolerance test (MMTT) before (Pre) and after (Post) exercise training. *Significantly different from Pre; $\mathrm{p}<0.05$. 
Individual subject responses for glucose iAUC and insulin and c-peptide AUC are shown in Figure 6. Notably, there was a great deal of variability between subjects in glucose iAUC, with five subjects showing a decrease in the postprandial glucose response following exercise training and five subjects showing an increase. Because of this, an exploratory non-parametric analysis was conducted to determine if factors could be identified which might explain differences between "Responders" (those who decreased glucose iAUC following our exercise training intervention) and "Nonresponders" (those who did not decrease glucose iAUC following our exercise training intervention). Pearson's product moment correlations were determined for \% change in glucose iAUC and descriptive data for all subjects, including BMI, \% body fat, the homeostatic model of insulin resistance (HOMA-IR), $\mathrm{VO}_{2 \text { peak }}$, fasting glucose, $\mathrm{HbA1c}$, age, and duration of diabetes. Additionally, subjects were divided into Responders and Non-responders based on whether glucose iAUC was higher in the Pre condition (Responders; $\mathrm{n}=5$ ) or in the Post condition (Non-responders; $\mathrm{n}=5$ ), and biserial correlations and t-tests were used to determine variables which might differentiate Responders from Non-responders. Results of the raw data correlations showed a trend for a relationship between $\%$ change in glucose iAUC and $\mathrm{HbA1c}(\mathrm{r}=0.522 ; \mathrm{p}=0.06)$ with greater reductions in glucose iAUC trending to associate with lower HbAlc levels, Additionally, there was a trend for a relationship between $\%$ change in glucose iAUC and fasting glucose concentrations $(r=0.426 ; p=0.11)$ with lower fasting glucose concentrations seen with greater reductions in glucose iAUC. Biserial correlation analysis of Responders and Non-responders indicated a significant relationship for $\mathrm{HbA1c}(\mathrm{r}=$ $0.61 ; \mathrm{p}<0.05)$ with Responders generally having lower levels compared to Non- 
responders. Similarly, the t-test showed a trend for Responders to exhibit lower HbA1c vs. Non-responders $(6.5 \pm 0.1 \%$ vs $7.0 \pm 0.2 \% ; p=0.08)$. The biserial correlation analysis also revealed that age was significantly correlated with the response $(r=0.62 ; p<0.05)$, with the t-test confirming that Responders were significantly older compared to Nonresponders (62 \pm 2 years vs. $54 \pm 3$ years, respectively; $\mathrm{p}<0.05)$. 
A

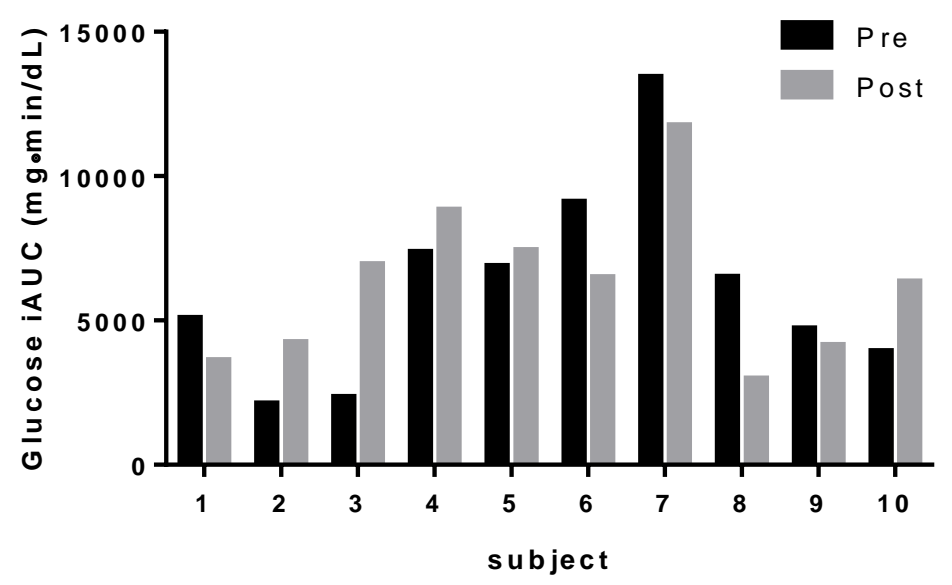

B

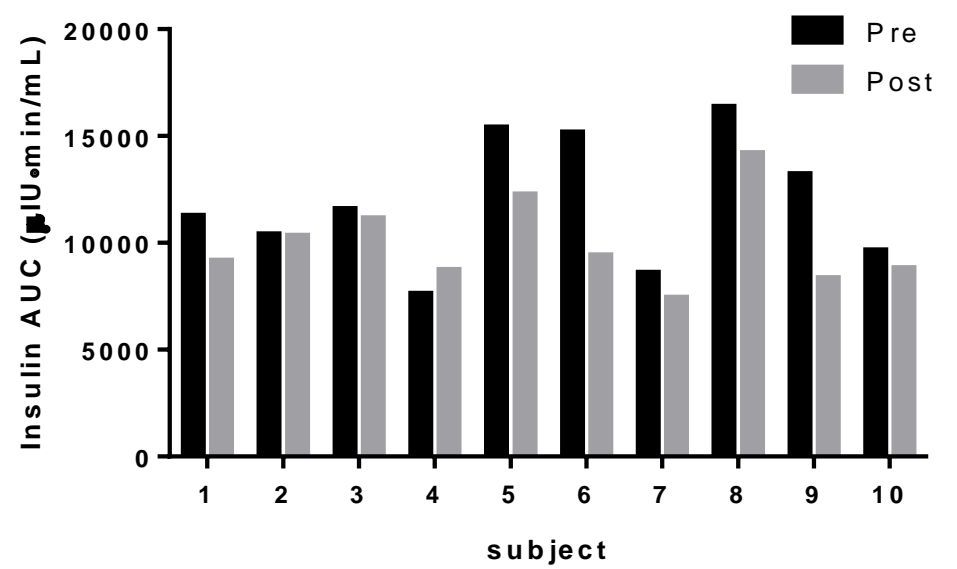

C

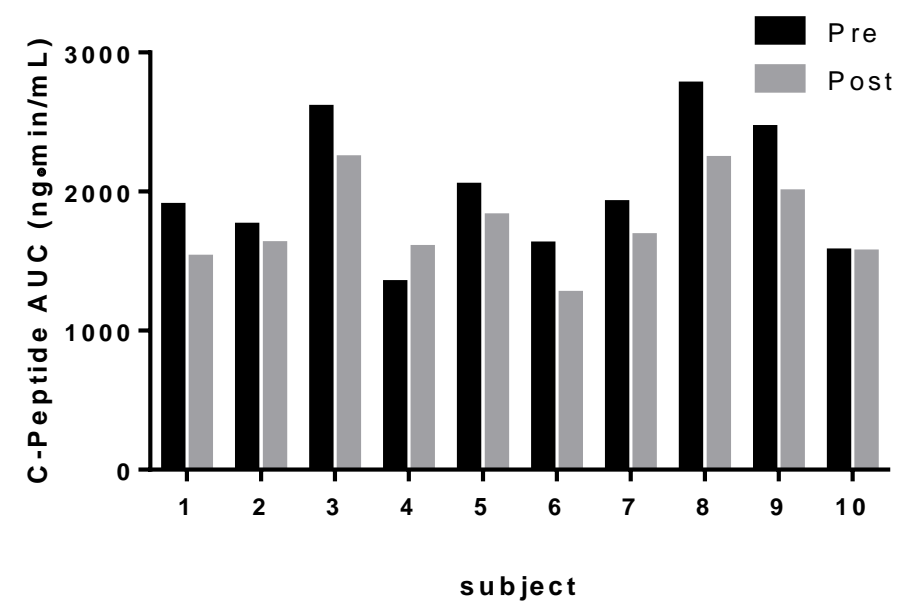

FIGURE 6 - Individual subject responses for glucose (A), insulin (B), and c-peptide

(C) following a mixed meal tolerance test (MMTT) before (Pre) and after (Post) exercise training. 


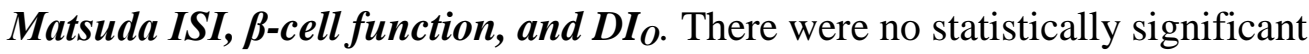

differences Pre vs. Post exercise training for Matsuda ISI, $\Delta$ glucose $_{(30-0)} / \Delta$ c-peptide $(30-$ 0), or $\mathrm{DI}_{\mathrm{O}}$. However, there was a $20 \%$ increase in the Matsuda ISI following exercise training that trended towards statistical significance $(\mathrm{p}<0.10) \quad($ Figure 7). 

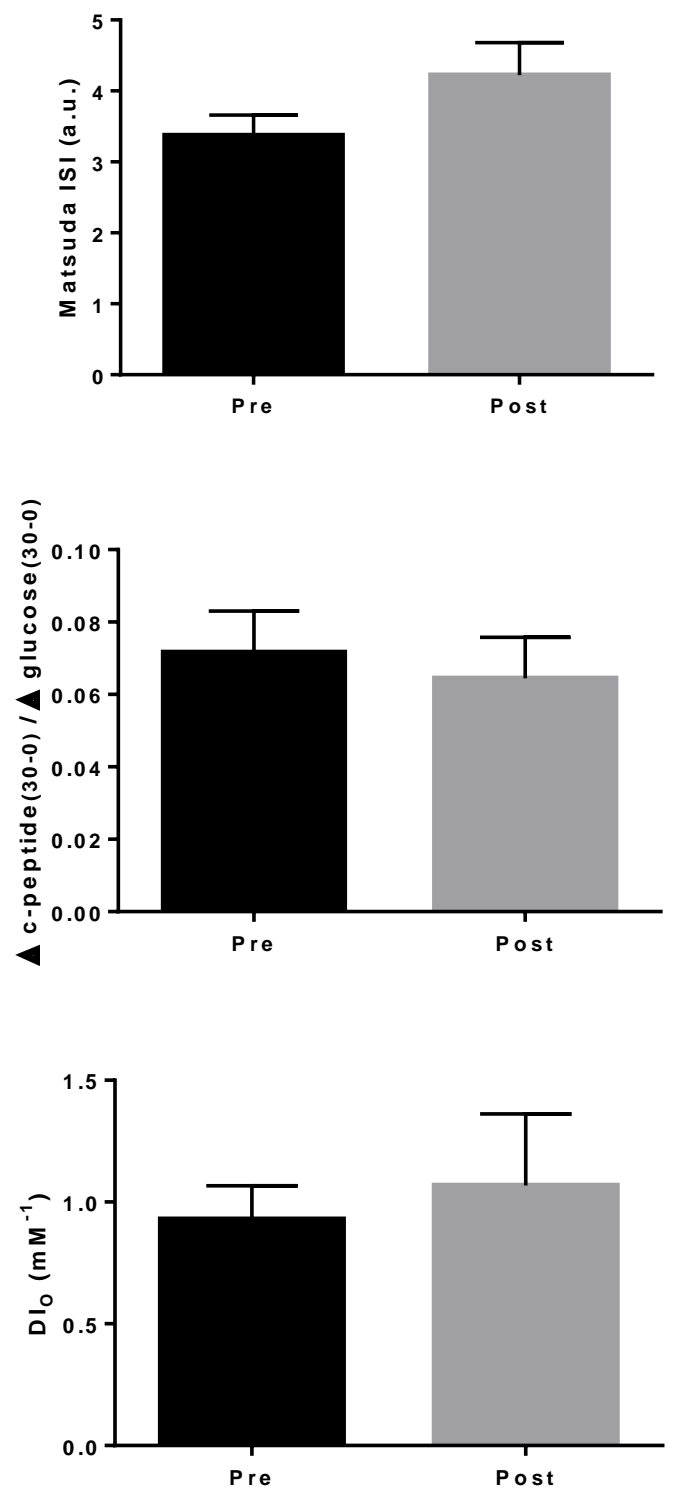

FIGURE 7 - Calculations for the Matsuda Insulin Sensitivity Index (Matsuda ISI), $\beta$-cell function ( $\Delta$ c-peptide $(30-0) / \Delta$ glucose $\left._{(30-0)}\right)$, and the oral disposition index $\left(\mathrm{DI}_{\mathrm{O}}\right)$ derived from the MMTT before (Pre) and after (Post) exercise training. 
Tracer kinetic modeling outcomes. There was no difference in $\mathrm{Ra}_{\text {total }}$ (calculated using the TTR from the infused $\left[6,6-{ }^{2} \mathrm{H}_{2}\right]$ glucose isotope) between the Pre and Post conditions (Figure 8). Because using calculations of the entire glucose sum (glucose from pure glucose, from sucrose, and from starch) for the meal produced nonphysiological values in kinetic modeling for $\mathrm{Ra}_{\text {oral }}, \mathrm{EGP}$, and $\mathrm{Rd}, \mathrm{Ra}_{\text {total }}$ is the only outcome that can be reported numerically with scientific validity. However, because the only alterable variables included in the equations for $\mathrm{Ra}_{\text {oral }}$, EGP, and $\mathrm{Rd}$ remained unchanged (Figure 9; no differences in tracer to TTR for $\left[\mathrm{U}_{-}{ }^{13} \mathrm{C}_{6}\right]$ glucose Pre/Post or for $\left[6,6-{ }^{2} \mathrm{H}_{2}\right]$ glucose Pre/Post, verified by paired sample t-tests; $\left.\mathrm{p}>0.05\right)$, we can robustly conclude that these outcomes were not changed by our intervention. 

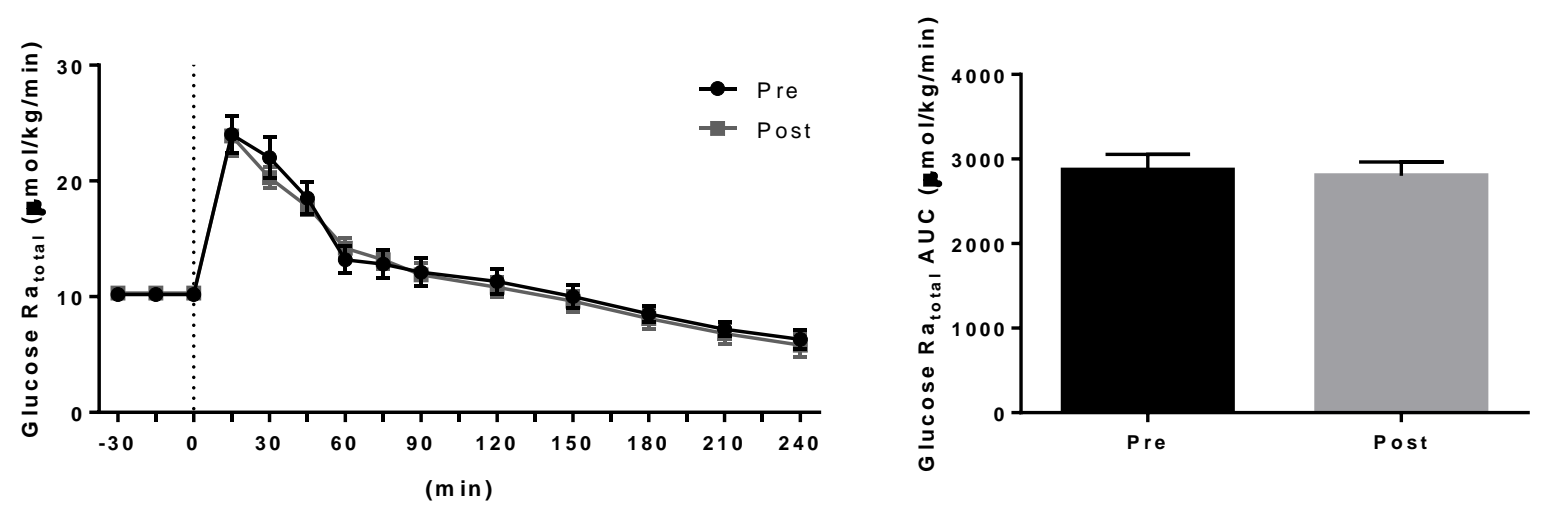

FIGURE 8 - Total glucose rate of appearance ( $\left(\mathrm{a}_{\text {total }}\right)$ was not different between the Pre and Post exercise training conditions $(p<0.05)$. 
A

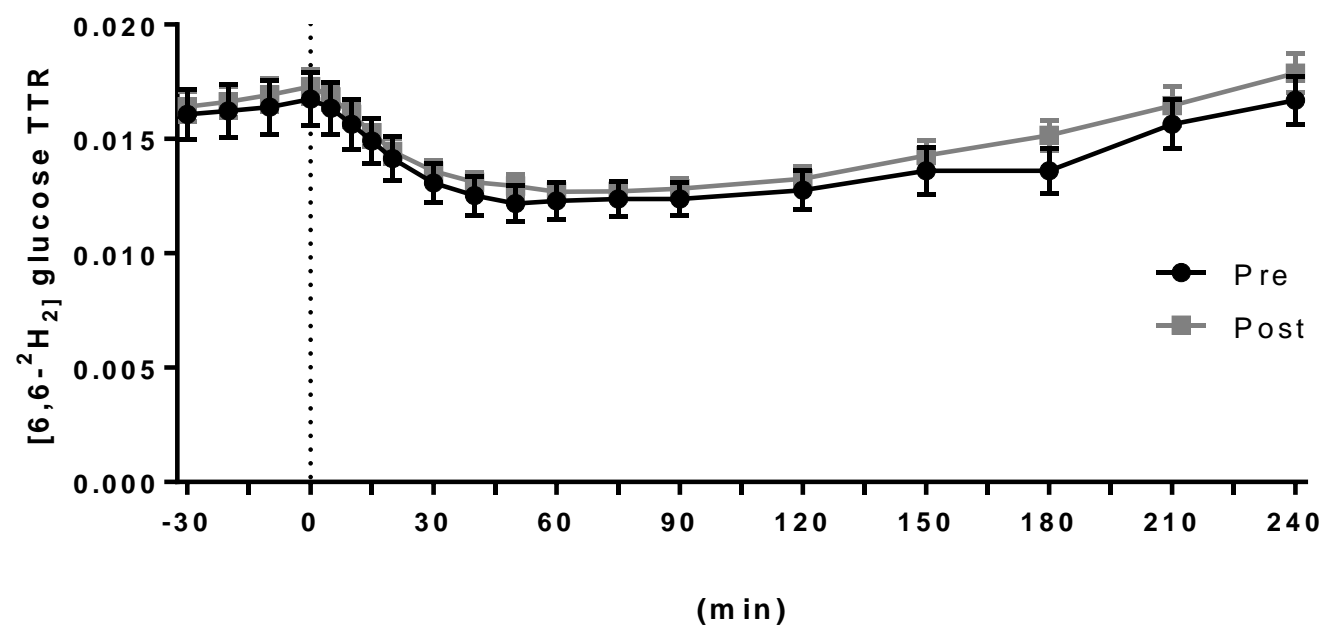

B

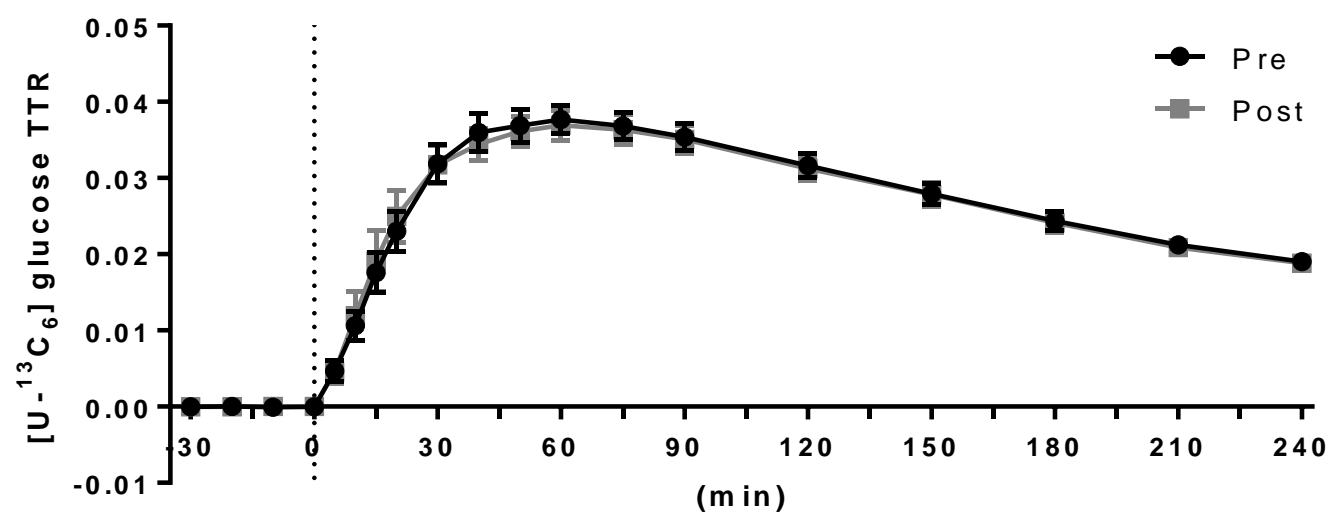

FIGURE 9 - The TTR across the four hour postprandial time course was not different between the Pre and Post exercise training conditions for either $\left[6,6-{ }^{2} \mathrm{H}_{2}\right]$ glucose (A) or $\left[\mathrm{U}^{13} \mathrm{C}_{6}\right]$ glucose (B). This was verified by paired samples t-tests comparing Pre vs. Post AUC for each TTR (p < 0.05). 


\section{Discussion}

The primary findings of this study were that one week of aerobic exercise training in subjects with T2D improved insulin sensitivity (reduction in the postprandial insulin response with no change in glucose concentration; trend for improved Matsuda ISI) but did not improve PPG. There also were no changes in the TTR for either of the glucose isotopes $\left(\left[\mathrm{U}_{-}{ }^{13} \mathrm{C}_{6}\right]\right.$ glucose or $\left[6,6-{ }^{2} \mathrm{H}_{2}\right]$ glucose $)$; thus, we conclude that there was no change in Ra, EGP, or Rd. Though glucose iAUC was not reduced following a week of aerobic exercise training, and there were no changes in measures of PPG as assessed by CGMS, both insulin and c-peptide AUC were reduced. The same postprandial glucose concentrations with less of an insulin response (indicated by the reduction in c-peptide concentrations) and a lower circulating insulin concentration is suggestive of improved insulin sensitivity, and this also is reflected in the $20 \%$ change in the Matsuda ISI.

The reductions in postprandial insulin and c-peptide AUC following the intervention, with no change in postprandial glucose concentrations, indicate an improvement in insulin sensitivity. This is in agreement with previous work from our lab examining responses to an OGTT following seven days of exercise training (45).In contrast to the study by Mikus et al. (45), though, the Matsuda ISI, a common calculation of insulin sensitivity, was not changed in the current study, likely due to a difference in the statistical models employed. The current study examined several dependent variables and thus applied a Bonferroni correction to reduce the probability of type 1 statistical error.(7). In contrast, the previous study used a paired-samples t-test but did not apply a Bonferroni correction since the Matsuda ISI was the only outcome assessed. The Bonferroni adjustment method is notably conservative, and because of this there is an 
increased risk of type 2 errors, particularly in studies with smaller subject populations (7). In spite of this, though, it is recommended to use the correction when multiple dependent variables are assessed since a type 2 statistical error is considered a lesser violation than a type 1 error $(7,25)$. If the same statistical method used by Mikus et al. $(45)$ were to be applied to the present data, the result of the physiologically relevant $20 \%$ change would be statistically significant at $\mathrm{p}<0.05$. Another potential reason a significant difference was not observed in the calculated Matsuda ISI could be the high degree of variability seen in this study with a relatively low number of subjects. Subjects included in the present study were on a variety of different medications, were of mixed sex, spanned a wide age range, and reported having diabetes over a wide duration range. Thus, while it is important to study diabetic subjects across the ranges of all of these variables, it is possible that the present study was underpowered to detect changes in insulin sensitivity indices in such a heterogeneous diabetic population. Considering the possibility of statistical errors, a $20 \%$ increase in insulin sensitivity, which is in agreement with other literature examining exercise training and postprandial glycemic control $(40,45,51)$, is considered clinically relevant.

Another interesting finding of the current study was that insulin concentrations (insulin AUC) and a marker of the insulin response (c-peptide AUC) were both reduced in the presence of unchanged hyperglycemia. It is somewhat counterintuitive that the insulin response would be decreased in the presence of unchanged postprandial hyperglycemia. Although this change indicates increased insulin sensitivity, as there was the same concentration of glucose for less insulin, one might expect that the initial response would be for glucose to be reduced. However, this is not the only study to have 
observed this phenomenon $(20,45,46)$. As mentioned previously, Mikus et al. (45) also found that insulin sensitivity was improved but glucose unchanged, in response to an OGTT before and after seven days of exercise training. In the study by Mikus, the change in the Matsuda ISI was driven by a trend for lower insulin following exercise training, but the difference in insulin was not as pronounced as in the present study. This discrepancy (significance in Matsuda ISI but not insulin in previous study; significance in insulin AUC but not Matsuda ISI in present study) could be due to differences in glucose concentrations, though the differences were not significant in either study.

Similar to these studies, Cononie et al. (20) reported in older non-diabetic men and women that insulin responses also were reduced while glucose concentration went unchanged in response to an OGTT following seven days of exercise training. Likewise, our similar observation in subjects with T2D in response to a meal test is consistent with what is seen in relatively healthy older adults, a population that often exhibits hyperinsulinemia (32). As already stated, the reduction in postprandial insulin with no change in glucose concentrations, in both studies, demonstrates improved insulin sensitivity, and it is possible that this early adaptation of reducing hyperinsulinemia would translate to reduced circulating blood glucose with continued training. In this same study, Cononie and colleagues also examined glucose and insulin responses to an OGTT after just one day of exercise and found no changes. Thus, it appears that seven days of exercise training produces adaptations beyond what is observed with a single exercise bout in healthy individuals, suggesting a time course for improvements in factors influencing postprandial glycemic control. However, because the subjects in the study by 
Cononie and colleagues were comparatively healthy, there could be less room for improvement in PPG than in subjects with T2D if chronic hyperglycemia is not present.

One of the first studies to utilize the seven day exercise model to examine changes in postprandial glycemic control was from John Holloszy's lab and performed in subjects with T2D. In this study, Rogers et al. (51) found that both insulin and glucose responses to an OGTT were reduced following seven days of aerobic exercise training but not after just one day of exercise. However, in the present study, we did not see reduced PPG following a week of exercise training. This could be due to a number of differences between the two studies. First, Rogers, et al. utilized a 100 gram glucose beverage to assess PPG, while the meal used in the present study contained 60 grams of carbohydrate from mixed sources as part of a meal. Because of the large (40\%) difference in total carbohydrate, as well as differences in digestion and absorption rates of starch compared to simple sugars and of mixed macronutrient meals compared to simple sugars $(34,35$, 64), there were likely large differences in absorption of glucose from the two different challenges. Although similar in total energy ( 400 kcal), overall blood glucose concentrations would likely be higher with the 100 gram glucose beverage compared to the mixed meal, since $100 \%$ of the energy from the beverage was from carbohydrate, compared to $60 \%$ from the meal. This greater glucose challenge could theoretically contribute to a more severe detriment in glucose uptake in the sedentary condition and therefore more room to show improvement with exercise training.

Another difference between the present study and that of Rogers et al. (51) is how exercise training was performed. The present study utilized the same intensity and duration (60\% of HRR for 60 minutes) every day for seven consecutive days. Rogers et 
al. utilized a more intense, inverse taper protocol in which subjects exercised for 60 minutes at moderate intensity (60\% maximal heart rate) for the first four days but then increased the intensity on the last three days to $68 \%$ of $\mathrm{VO}_{2 \max }$, with a 10 minute break halfway through the exercise session. Thus, the effects shown by Rogers et al. are responses to more vigorous intensity exercise than what is currently recommended by ACSM and the American Diabetes Association $(18,19)$. It is possible that the increased exercise intensity resulted in greater improvements than what would be seen with a more moderate intensity training protocol. Further, differences in subjects' ages, duration of diabetes, and medications might also contribute to differences in the observed outcomes.

Although the aforementioned studies found differences in PPG following exercise training when assessed by OGTT, there are several outlined differences between those studies and the present methodology and subjects utilized. Further, other studies examining the effects of a week of exercise on PPG have shown improved insulin sensitivity but no change in glucose responses to an OGTT $(37,45)$. Thus, while in contrast to our hypothesis, our observation that PPG was not improved following seven days of exercise training in response to a MMTT is not inconceivable.

There are also other potential explanations for why subjects in this study did not respond as robustly to exercise training as in previous studies. T2D is a heterogeneous diagnosis, with varying degrees of multi-tissue pathology that may cause differences in how subjects respond to different treatments, including exercise $(10,24)$. As shown by Solomon et al. (56), there is great inter-subject variability in changes in glycemic control in response to exercise training in individuals with impaired glucose tolerance and T2D, and differences in insulin secretion and $\beta$-cell function appear to contribute greatly to 
how subjects respond. In the study by Solomon et al., only $2 / 3$ of subjects with exerciseinduced improvements in $\mathrm{VO}_{2 \max }$ and body composition, following 12-16 weeks of aerobic exercise training, exhibited improved glycemic control when assessed by HbA1c, fasting glucose, or response to an oral glucose challenge. Factors determined to predict whether or not a subject would respond to exercise training were a high fasting glucose concentration pre-training and low pre-training glucose-stimulated insulin secretory capacity. Together, this suggests that those patients with more advanced or less controlled T2D may not respond to exercise interventions as readily as those who exhibit more normal pancreatic $\beta$-cell function and lower fasting glucose levels.

While we hypothesized that PPG would be reduced following the one week aerobic exercise training intervention, we instead saw reduced insulin concentrations without a reduction in PPG. While in contrast to what we expected, this outcome is supported mechanistically by work in cell culture. Galbo et al. (3) exposed primary pancreatic islets isolated from exercise trained rats to physiological glucose concentrations and found that compared to control islets from sedentary rats, the islets from exercised rats secreted less insulin. They attributed this to reduced glucose sensitivity, which could explain the lower insulin concentrations observed with exercise training. Because less insulin is needed for a given glucose concentration, less is secreted from the pancreas.

Yet, even in the absence of a change in PPG following our exercise training intervention, the reduction in insulin concentrations in physiologically important. While previous dogma insisted that hyperglycemia was the cause of hyperinsulinemia, such that insulin levels increased as a response to high circulating glucose, newer evidence 
suggests that the opposite may be true and that hyperinsulinemia is a cause of hyperglycemia rather than an effect $(21,49,52)$. Thus, reducing hyperinsulinemia early in the course of exercise training, as seen in this study, could be a mechanism to drive down PPG with continued training. As reviewed by Pories and Dohm (49), studies on patients with T2D who undergo bariatric surgery show these patients to have a resolution of the disease with complete early reversal of hyperinsulinemia to concentrations observed in lean control subjects. Mechanistically, Pories and Dohm hypothesize that the decreased insulin leads to a decrease in the production of gluconeogenic substrate (e.g., lactate) and less Cori cycling, ultimately reducing hyperglycemia. As reviewed by Corkey (21), work in animals and cell culture has shown that several environmental factors and food additives, including monoacylglycerides, artificial sweeteners, and iron, increase basal insulin secretion irrespective of glucose, most likely via increasing reactive oxygen species (ROS). With repeated exposure to increased ROS, it is likely that insulin hypersecretion would lead to insulin resistance, further potentiating the need for more insulin to be secreted to maintain glycemia (52). In view of the emerging datadriven hypothesis that hyperinsulinemia drives hyperglycemia $(21,49,52)$, the early reduction in circulating insulin with exercise training is viewed as a beneficial adaptation. Further, hyperinsulinemia has been linked to several morbidities including hypertension (23), Alzheimer disease (42), and various cancers $(3,65)$, and hyperinsulinemia even with normal glucose tolerance has been tied to increased coronary artery disease (66) . Thus, a reduction in insulin concentrations, even without a change in glucose concentrations, may be advantageous. 
Consistent with our hypothesis, $\beta$-cell function was not changed in this study. As $\beta$-cell function and insulin sensitivity are the two factors influencing the disposition index, it is not surprising that $\mathrm{DI}_{\mathrm{O}}$ also was unchanged with this model of short-term exercise training. Although insulin sensitivity was improved when assessed by examining the reduction in insulin with no change in glucose, $\beta$-cell function remained unchanged following seven days of training.. However, improvements in the disposition index have been shown utilizing longer exercise training interventions (56). Thus, the results of the present study, when viewed in light of previous work, indicate that short-term, moderate intensity aerobic exercise training is not enough of a training stimulus to improve $\mathrm{DI}_{\mathrm{O}}$, primary due to the lack of change in $\beta$-cell function.

Although previous studies using CGMS have found short-term exercise training to improve PPG and PPGE $(46,48)$, this study found no differences in any of the measures of PPG or PPGE examined. A major limitation of the current study is the small sample size included in CGMS analyses, and it is possible that this could affect the outcomes. Another potential explanation is the extreme variability commonly observed with CGMS devices, which could further potentiate the problem of having a small sample size. Even considering the small sample size, however, individual subject responses were similar, and it is unlikely that adding more subjects would change the results. Finally, though manufacturer recommendations were followed, the monitors did not record data for several time blocks. Other studies have had similar problems, and to combat this, some researchers recommend using two separate monitors on the same subject, at the same time (secondary reports and oral discussions). This would prove complicated, 
though, as the monitors may not provide the same data and choosing which data to use at which times would introduce added subjectivity into the analysis.

A major limitation of this study was the unforeseen violation of the assumption that the glucose tracer used in the meal $\left(\left[\mathrm{U}_{-}-{ }^{13} \mathrm{C}_{6}\right]\right.$ glucose $)$ would be metabolically undetectable from all of the glucose consumed in the meal, particularly the starch portion in the tortilla. Both pure glucose from the meal and glucose derived from the rapid dissociation of glucose from sucrose should theoretically be traced effectively by the oral glucose isotope; but, glucose derived from starch in the meal would be digested more slowly and thus would not appear as rapidly as the tracer (8). This oversight was realized when calculating $\mathrm{Ra}_{\text {oral }}$ provided negative, non-physiological values. Thus, several of the primary outcomes $\left(\mathrm{Ra}_{\text {oral }}, \mathrm{EGP}\right.$, and $\left.\mathrm{Rd}\right)$ for mechanistic explanation could not be reported with numeric validity. However, the redeeming quality of this particular study is that ultimately, it did not matter since there were no differences in TTR for the orally consumed isotope. Because there were no differences in TTR for either glucose isotope, and this is the only variable that differed in the Pre/Post design, we can robustly conclude, in the face of problems in kinetic analysis, that there was no change in $\mathrm{Ra}_{\text {oral }}$, EGP, or Rd. Even so, this should be avoided in the future by altering the meal so that all ingested glucose is provided in a form that is metabolically undetectable from the tracer. Although providing all carbohydrate in the form of glucose might detract slightly from the conceptual validity of using a mixed meal, the outcomes would be more meaningful and thus provide stronger data. And in fact, this has been done previously (28).

The conclusion that EGP and Rd did not change was in contrast to our hypothesis. We hypothesized that Rd (glucose disposal rate) would be increased due to increased 
glucose uptake primarily by skeletal muscle. We also hypothesized that EGP would be decreased following exercise training, since short-term exercise training has previously shown enhanced suppression of EGP when assessed by hyperinsulinemic euglycemic clamp (40). Nonetheless, these results coincide with the observation that glucose iAUC was not changed. If the postprandial glucose response did not change following the intervention, it is not surprising that the mechanisms purported to influence the hypothesized change also would not differ.

\section{Conclusion}

In summary, while the free-living measures of PPG and PPGE from CGMS were not changed and postprandial glucose concentrations were not affected following the laboratory-based MMTT, insulin sensitivity, when considering the change in insulin AUC with no change in glucose iAUC, was improved, indicative of an underlying change in postprandial glycemic control mechanisms. However, since glucose concentration was not lowered following the exercise intervention in this study, it is not surprising that the hypothesized mechanisms of reduced PPG (increased glucose disposal and reduced EGP) were not changed. The results of this study suggest that one week of aerobic exercise training does not lower postprandial glucose responses to a physiological mixed meal test, but it does improve insulin sensitivity. 


\section{References}

1. American College of Sports Medicine. ACSM's Guidelines for Exercise Testing and Prescription. 9th ed.: Lippincott Williams \& Wilkins; 2013.

2. American Diabetes Association. Standards of medical care in diabetes--2014. Diabetes Care. 2014;37 Suppl 1:S14-80.

3. Argiles JM, Lopez-Soriano FJ. Insulin and cancer (Review). International Journal of Oncology. 2001;18(4):683-7.

4. Armstrong MJ, Boule NG, Sigal RJ. Exercise interventions and glycemic control in patients with diabetes. JAMA. 2011;306(6):607; author reply 8-9.

5. Avogaro A. Postprandial glucose: marker or risk factor? Diabetes Care. 2011;34(10):2333-5.

6. Bantle JP, Laine DC, Castle GW, Thomas JW, Hoogwerf BJ, Goetz FC. Postprandial glucose and insulin responses to meals containing different carbohydrates in normal and diabetic subjects. The New England Journal of Medicine. 1983;309(1):7-12.

7. Bland JM, Altman DG. Multiple significance tests: the Bonferroni method. British Medical Journal. 1995;310(6973):170.

8. Borgstrom B, Dahlqvist A, Lundh G, Sjovall J. Studies of intestinal digestion and absorption in the human. The Journal of Clinical Investigation. 1957;36(10):1521-36.

9. Boulay MR, Simoneau JA, Lortie G, Bouchard C. Monitoring high-intensity endurance exercise with heart rate and thresholds. Medicine and Science in Sports and Exercise. 1997;29(1):125-32. 
10. Boule NG, Weisnagel SJ, Lakka TA et al. Effects of exercise training on glucose homeostasis: the HERITAGE Family Study. Diabetes Care. 2005;28(1):108-14.

11. Bradley D, Magkos F, Eagon JC et al. Matched weight loss induced by sleeve gastrectomy or gastric bypass similarly improves metabolic function in obese subjects. Obesity. 2014;22(9):2026-31.

12. Brouns F, Bjorck I, Frayn KN et al. Glycaemic index methodology. Nutrition Research Reviews. 2005;18(1):145-71.

13. Cavalot F, Pagliarino A, Valle M et al. Postprandial blood glucose predicts cardiovascular events and all-cause mortality in type 2 diabetes in a 14-year follow-up: lessons from the San Luigi Gonzaga Diabetes Study. Diabetes Care. 2011;34(10):2237-43.

14. Cavalot F, Petrelli A, Traversa M et al. Postprandial blood glucose is a stronger predictor of cardiovascular events than fasting blood glucose in type 2 diabetes mellitus, particularly in women: lessons from the San Luigi Gonzaga Diabetes Study. The Journal of Clinical Endocrinology and Metabolism. 2006;91(3):813-9.

15. Centers for Disease Control and Prevention. National Diabetes Statistics Report. 2014.

16. Ceriello A, Esposito K, Piconi L et al. Oscillating glucose is more deleterious to endothelial function and oxidative stress than mean glucose in normal and type 2 diabetic patients. Diabetes. 2008;57(5):1349-54.

17. Ceriello A, Hanefeld M, Leiter L et al. Postprandial glucose regulation and diabetic complications. Archives of Internal Medicine. 2004;164(19):2090-5. 
18. Colberg SR, Albright AL, Blissmer BJ et al. Exercise and type 2 diabetes: American College of Sports Medicine and the American Diabetes Association: joint position statement. Exercise and type 2 diabetes. Medicine and Science in Sports and Exercise. 2010;42(12):2282-303.

19. Colberg SR, Sigal RJ, Fernhall B et al. Exercise and type 2 diabetes: the American College of Sports Medicine and the American Diabetes Association: joint position statement executive summary. Diabetes Care. 2010;33(12):2692-6.

20. Cononie CC, Goldberg AP, Rogus E, Hagberg JM. Seven consecutive days of exercise lowers plasma insulin responses to an oral glucose challenge in sedentary elderly. Journal of the American Geriatrics Society. 1994;42(4):394-8.

21. Corkey BE. Banting lecture 2011: hyperinsulinemia: cause or consequence? Diabetes. 2012;61(1):4-13.

22. Dall TM, Yang W, Halder P et al. The economic burden of elevated blood glucose levels in 2012: diagnosed and undiagnosed diabetes, gestational diabetes mellitus, and prediabetes. Diabetes Care. 2014;37(12):3172-9.

23. DeFronzo RA. The effect of insulin on renal sodium metabolism. A review with clinical implications. Diabetologia. 1981;21(3):165-71.

24. Defronzo RA. Banting Lecture. From the triumvirate to the ominous octet: a new paradigm for the treatment of type 2 diabetes mellitus. Diabetes. 2009;58(4):77395.

25. Field AP. Discovering statistics using SPSS : (and sex and drugs and rock ' $n$ ' roll). 3rd ed. Los Angeles: SAGE; 2009, xxxiii, 821 pages. 
26. Gao J, Ren J, Gulve EA, Holloszy JO. Additive effect of contractions and insulin on GLUT-4 translocation into the sarcolemma. Journal of Applied Physiology. 1994;77(4):1597-601.

27. Garber CE, Blissmer B, Deschenes MR et al. American College of Sports Medicine position stand. Quantity and quality of exercise for developing and maintaining cardiorespiratory, musculoskeletal, and neuromotor fitness in apparently healthy adults: guidance for prescribing exercise. Medicine and Science in Sports and Exercise. 2011;43(7):1334-59.

28. Gastaldelli A, Casolaro A, Pettiti M et al. Effect of pioglitazone on the metabolic and hormonal response to a mixed meal in type II diabetes. Clinical Pharmacology and Therapeutics. 2007;81(2):205-12.

29. Hanefeld M, Koehler C, Henkel E, Fuecker K, Schaper F, TemelkovaKurktschiev T. Post-challenge hyperglycaemia relates more strongly than fasting hyperglycaemia with carotid intima-media thickness: the RIAD Study. Risk Factors in Impaired Glucose Tolerance for Atherosclerosis and Diabetes. Diabetic Medicine : a Journal of the British Diabetic Association. 2000;17(12):835-40.

30. Hansen PA, Nolte LA, Chen MM, Holloszy JO. Increased GLUT-4 translocation mediates enhanced insulin sensitivity of muscle glucose transport after exercise. Journal of Applied Physiology. 1998;85(4):1218-22.

31. Hasson RE, Howe CA, Jones BL, Freedson PS. Accuracy of four resting metabolic rate prediction equations: effects of sex, body mass index, age, and race/ethnicity. Journal of Science and Medicine in Sport/Sports Medicine Australia. 2011;14(4):344-51. 
32. Holloszy JO, Schultz J, Kusnierkiewicz J, Hagberg JM, Ehsani AA. Effects of exercise on glucose tolerance and insulin resistance. Brief review and some preliminary results. Acta Medica Scandinavica. Supplementum. 1986;711:55-65.

33. IDF Diabetes Atlas Group. Update of mortality attributable to diabetes for the IDF Diabetes Atlas: estimates for the year 2011. Diabetes Research and Clinical Practice. 2013;100(2):277-9.

34. Jenkins DJ, Ghafari H, Wolever TM et al. Relationship between rate of digestion of foods and post-prandial glycaemia. Diabetologia. 1982;22(6):450-5.

35. Jenkins DJ, Wolever TM, Taylor RH et al. Glycemic index of foods: a physiological basis for carbohydrate exchange. The American Journal of Clinical Nutrition. 1981;34(3):362-6.

36. Kahn SE, Prigeon RL, McCulloch DK et al. Quantification of the relationship between insulin sensitivity and beta-cell function in human subjects. Evidence for a hyperbolic function. Diabetes. 1993;42(11):1663-72.

37. Kang J, Robertson RJ, Hagberg JM et al. Effect of exercise intensity on glucose and insulin metabolism in obese individuals and obese NIDDM patients. Diabetes Care. 1996;19(4):341-9.

38. Kant AK, Graubard BI. Secular trends in patterns of self-reported food consumption of adult Americans: NHANES 1971-1975 to NHANES 1999-2002. The American Journal of Clinical Nutrition. 2006;84(5):1215-23.

39. Karvonen J, Vuorimaa T. Heart rate and exercise intensity during sports activities. Practical application. Sports Medicine. 1988;5(5):303-11. 
40. Kirwan JP, Solomon TP, Wojta DM, Staten MA, Holloszy JO. Effects of 7 days of exercise training on insulin sensitivity and responsiveness in type 2 diabetes mellitus. American Journal of Physiology. Endocrinology and Metabolism. 2009;297(1):E151-6.

41. Little JP, Jung ME, Wright AE, Wright W, Manders RJ. Effects of high-intensity interval exercise versus continuous moderate-intensity exercise on postprandial glycemic control assessed by continuous glucose monitoring in obese adults. Applied Physiology, Nutrition, and Metabolism = Physiologie appliquee, nutrition et metabolisme. 2014;39(7):835-41.

42. Luchsinger JA, Tang MX, Shea S, Mayeux R. Hyperinsulinemia and risk of Alzheimer disease. Neurology. 2004;63(7):1187-92.

43. Malin SK, Solomon TP, Blaszczak A, Finnegan S, Filion J, Kirwan JP. Pancreatic beta-cell function increases in a linear dose-response manner following exercise training in adults with prediabetes. American Journal of Physiology. Endocrinology and Metabolism. 2013;305(10):E1248-54.

44. Matsuda M, DeFronzo RA. Insulin sensitivity indices obtained from oral glucose tolerance testing: comparison with the euglycemic insulin clamp. Diabetes Care. 1999;22(9):1462-70.

45. Mikus CR, Fairfax ST, Libla JL et al. Seven days of aerobic exercise training improves conduit artery blood flow following glucose ingestion in patients with type 2 diabetes. Journal of Applied Physiology. 2011;111(3):657-64. 
46. Mikus CR, Oberlin DJ, Libla J, Boyle LJ, Thyfault JP. Glycaemic control is improved by 7 days of aerobic exercise training in patients with type 2 diabetes. Diabetologia. 2012;55(5):1417-23.

47. Mikus CR, Oberlin DJ, Libla JL, Taylor AM, Booth FW, Thyfault JP. Lowering physical activity impairs glycemic control in healthy volunteers. Medicine and Science in Sports and Exercise. 2012;44(2):225-31.

48. Oberlin DJ, Mikus CR, Kearney ML et al. One bout of exercise alters free-living postprandial glycemia in type 2 diabetes. Medicine and Science in Sports and Exercise. 2014;46(2):232-8.

49. Pories WJ, Dohm GL. Diabetes: have we got it all wrong? Hyperinsulinism as the culprit: surgery provides the evidence. Diabetes Care. 2012;35(12):2438-42.

50. Pruessner JC, Kirschbaum C, Meinlschmid G, Hellhammer DH. Two formulas for computation of the area under the curve represent measures of total hormone concentration versus time-dependent change. Psychoneuroendocrinology. 2003;28(7):916-31.

51. Rogers MA, Yamamoto C, King DS, Hagberg JM, Ehsani AA, Holloszy JO. Improvement in glucose tolerance after $1 \mathrm{wk}$ of exercise in patients with mild NIDDM. Diabetes Care. 1988;11(8):613-8.

52. Shanik MH, Xu Y, Skrha J, Dankner R, Zick Y, Roth J. Insulin resistance and hyperinsulinemia: is hyperinsulinemia the cart or the horse? Diabetes Care. 2008;31 Suppl 2:S262-8. 
53. Sigal RJ, Kenny GP, Boule NG et al. Effects of aerobic training, resistance training, or both on glycemic control in type 2 diabetes: a randomized trial. Annals of Internal Medicine. 2007;147(6):357-69.

54. Singer DE, Nathan DM, Anderson KM, Wilson PW, Evans JC. Association of HbA1c with prevalent cardiovascular disease in the original cohort of the Framingham Heart Study. Diabetes. 1992;41(2):202-8.

55. Solomon TP, Haus JM, Kelly KR, Rocco M, Kashyap SR, Kirwan JP. Improved pancreatic beta-cell function in type 2 diabetic patients after lifestyle-induced weight loss is related to glucose-dependent insulinotropic polypeptide. Diabetes Care. 2010;33(7):1561-6.

56. Solomon TP, Malin SK, Karstoft K, Kashyap SR, Haus JM, Kirwan JP. Pancreatic beta-cell function is a stronger predictor of changes in glycemic control after an aerobic exercise intervention than insulin sensitivity. The Journal of Clinical Endocrinology and Metabolism. 2013;98(10):4176-86.

57. Sonnenberg GE, Keller U. Sampling of arterialized heated-hand venous blood as a noninvasive technique for the study of ketone body kinetics in man. Metabolism: Clinical and Experimental. 1982;31(1):1-5.

58. Steele R. Influences of glucose loading and of injected insulin on hepatic glucose output. Annals of the New York Academy of Sciences. 1959;82:420-30.

59. Stratton IM, Adler AI, Neil HA et al. Association of glycaemia with macrovascular and microvascular complications of type 2 diabetes (UKPDS 35): prospective observational study. British Medical Journal. 2000;321(7258):40512. 
60. Swain DP, Leutholtz BC. Heart rate reserve is equivalent to \% VO2 reserve, not to \%VO2max. Medicine and Science in Sports and Exercise. 1997;29(3):410-4.

61. Temelkova-Kurktschiev TS, Koehler C, Henkel E, Leonhardt W, Fuecker K, Hanefeld M. Postchallenge plasma glucose and glycemic spikes are more strongly associated with atherosclerosis than fasting glucose or HbA1c level. Diabetes Care. 2000;23(12):1830-4.

62. Utzschneider KM, Prigeon RL, Faulenbach MV et al. Oral disposition index predicts the development of future diabetes above and beyond fasting and 2-h glucose levels. Diabetes Care. 2009;32(2):335-41.

63. Wolever TM, Chiasson JL, Csima A et al. Variation of postprandial plasma glucose, palatability, and symptoms associated with a standardized mixed test meal versus $75 \mathrm{~g}$ oral glucose. Diabetes Care. 1998;21(3):336-40.

64. Wolever TM, Jenkins DJ. The use of the glycemic index in predicting the blood glucose response to mixed meals. The American Journal of Clinical Nutrition. 1986;43(1):167-72.

65. Yoon YS, Keum N, Zhang X, Cho E, Giovannucci EL. Hyperinsulinemia, insulin resistance and colorectal adenomas: A meta-analysis. Metabolism: Clinical and Experimental. 2015.

66. Zavaroni I, Bonora E, Pagliara $\mathrm{M}$ et al. Risk factors for coronary artery disease in healthy persons with hyperinsulinemia and normal glucose tolerance. The New England Journal of Medicine. 1989;320(11):702-6. 


\section{STUDY TWO}

\section{ASSESSING POSTPRANDIAL GLYCEMIC CONTROL RESPONSES TO SHORT-TERM AEROBIC EXERCISE TRAINING IN TYPE 2 DIABETES: A COMPARISON OF MIXED MEAL TOLERANCE TESTING TO STANDARD ORAL GLUCOSE TOLERANCE TESTS}

\section{Introduction}

Postprandial glycemic control is a major problem in people with type 2 diabetes (T2D), with impaired postprandial glycemia (PPG) contributing to multiple diabetic comorbidities, including cardiovascular disease and death $(30,31,36,37,171)$. Since T2D is a worldwide problem which afflicts between 9 and 10 percent of the United States population alone (34), and people with T2D and impaired PPG have drastically increased mortality rates (168), it is important to determine effective treatment interventions to reduce PPG and improve postprandial glycemic control.

Exercise is effective in improving postprandial glycemic control, and the one week, short term exercise intervention has previously been used to demonstrate this (120, 121). Previously, Mikus et al. demonstrated that several measures of free-living PPG were improved following exercise training as assessed by continuous glucose monitors following a one week aerobic exercise training intervention (121), but the oral glucose tolerance test (OGTT) does not appear to be sensitive in detecting these differences (92, 120). 
The OGTT is a common laboratory-based measure, the most used challenge test in scientific and clinical diabetes literature, and the only challenge-based test used to diagnose and classify diabetes or risk for diabetes $(8,10)$. Despite the high clinical use of the OGTT, it is not characteristic of a human's free-living condition, as it does not represent what occurs when a person consumes a mixed meal containing carbohydrates, proteins, and fat. Instead, the OGTT shows the response to a large bolus of glucose alone, a challenge not typically seen in the free-living condition. Further, repeated OGTT testing in the same individuals has revealed inconsistent results $(118,119,127)$, which are attributed to its high glycemic index (193). Thus, a mixed meal tolerance test (MMTT) in which carbohydrates, protein, and fat are ingested in the form of a meal (in either solid or liquid composition), would more accurately illustrate the daily physiological response and thus be preferential in determining what changes occur when people eat normal meals in the free-living condition (30,193). Mimicing the typical meal situation would and add validity to the application of laboratory-based testing to the free-living condition. Since the OGTT is a more standard measure clinically, the MMTT should thus be compared to the OGTT to see how responses to exercise, a stimulus previously shown to improve insulin sensitivity and glucose handling, relate.

To the researcher's knowledge, no study has examined how the MMTT and the OGTT compare in assessing responses to exercise training, which is an indispensable lifestyle therapy for improving postprandial glycemic control. While the MMTT is a promising measure, additional research is necessary to substantiate it against the OGTT and to compare measures of glycemic control between the two tests in response to exercise in the same subjects. Thus, the purpose of this study was to compare measures of 
postprandial glycemic control from an OGTT and a MMTT before and after a short-term, one week aerobic exercise training intervention.

\section{Methods}

All protocols were approved by the Health Sciences Institutional Review Board (IRB) at the University of Missouri, and subjects provided written informed consent before participating in the study.

\section{Subjects}

Subjects $(n=10)$ included in this study were sedentary and low-activity $(\leq 1$ session/week of physical activity of 30 minutes or more and not employed in a physically active job or hobby), overweight to obese [body mass index (BMI) $25.0-37.0 \mathrm{~kg} / \mathrm{m}^{2}$ ], males and females with physician-diagnosed T2D. They were recruited in and around Columbia, MO, for participation in a different study (See "Study One”), with a subset completing additional testing which is described here. Subjects were older (48-67 years), non-smokers, had glycosylated hemoglobin (HbA1c) $<10 \%$, did not use exogenous insulin in their diabetes management, and reported no previous cardiac events. Additionally, all subjects were screened for other co-morbidities using a combination of questionnaires, a fasting blood sample, and a physician-monitored maximal exercise stress test with electrocardiography (ECG). Further, all participants were weight-stable ( $<5 \%$ weight change in previous 3 months), were not breakfast skippers and reported no other irregular dietary patterns, and reported no restrictions which would prevent them from engaging in structured aerobic exercise training. 
Subjects answered preliminary phone screening questions as approved by the IRB, and if initial qualification criteria were met, they met with study staff and were given detailed information on study procedures, risks, and benefits, and all initial questions were addressed. After deciding to participate and providing written informed consent, subjects enrolled in the study completed a medical history questionnaire, a dietary questionnaire, and a food and activity record before returning to the exercise physiology lab for further screening and preliminary testing. Following a 12 hour overnight fast, subjects returned to the lab for a fasting blood draw used to assess blood glucose, HbA1c, blood lipids [total cholesterol, low-density lipoprotein cholesterol (LDL-C), high-density lipoprotein cholesterol (LDL-C), and triglycerides (TAG)] and screen for underlying metabolic disease by a commercial laboratory, as done previously (121). Additionally, subjects underwent anthropometric assessment for height and weight during this visit and a dual energy $\mathrm{x}$-ray absorptiometry (DXA) scan (Hologic QDR 4500A; Hologic, Bedford, MA) for determination of body composition. On a subsequent visit, subjects underwent a physician-monitored maximal exercise stress test at the University of Missouri's Clinical Research Center (CRC). This test was performed on a treadmill using the Bruce Protocol in accordance with guidelines outlined by the American College of Sports Medicine (ACSM), as performed previously (5, 135). Heart rate, blood pressure, and respiratory gases were monitored throughout the test, and subjects were encouraged to continue to exhaustion or until they met three of the criteria for achieving a maximal exercise test as described by ACSM (5). Respiratory gases were measured and oxygen consumption $\left(\mathrm{VO}_{2}\right)$ assessed using a metabolic cart (ParvoMedics True One 2400 Metabolic Measurement System; ParvoMedics, Sandy, UT), and 
cardiovascular rates and rhythms were monitored using a 12-lead ECG (Quinton Q-Stress v3.5 Exercise Test Monitor; Quinton Cardiology, Inc./Burdick, Deerfield, WI). Peak oxygen consumption $\left(\mathrm{VO}_{2 \text { peak }}\right)$ was determined from this test, and a cardiologist reviewed ECG reports to ensure that subjects could safely participate in the exercise training program. After completion of the exercise stress test, there was a minimum one-week washout period before subjects began the study protocol to eliminate any residual effects of the testing exercise bout, during which time the subjects were asked to resume their normal habitual living.

\section{Experimental design}

The study design is outlined in Figure 10. Glucose, insulin, and c-peptide responses to a glucose bolus (OGTT) and a mixed meal (MMTT) were compared over four hours in the morning, following an overnight fast, before (Pre) and after (Post) one week of aerobic exercise training. Additionally, an estimate of insulin sensitivity was calculated for each condition (Pre and Post) using the Matsuda insulin sensitivity index (ISI) (115), and Pre and Post $\beta$-cell function was estimated by calculating the change in cpeptide over the first 30 minutes divided by the change in glucose over the same time.

Following completion of all screening procedures, subjects returned to the laboratory to pick up pack-out meals for the study's control diet (consumed 3 days prior to the MMTT, the day of the MMTT, and one day following the MMTT and prior to the OGTT). Subjects also received instruction for consumption and detailed recording of food consumption and meal timing during this visit. This controlled study diet was administered prior to testing for both the Pre (before exercise training) and Post (after exercise training) conditions. 


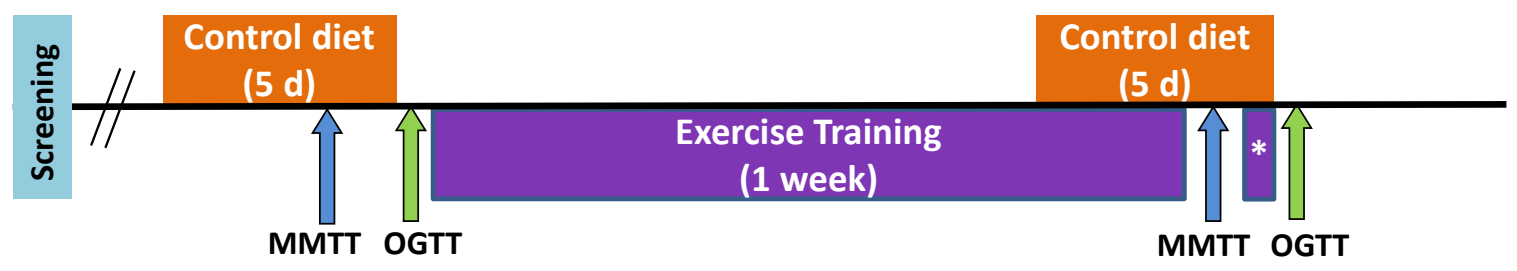

FIGURE 10 - Study design. A mixed meal tolerance test (MMTT) and an oral glucose tolerance test (OGTT) were separated by one day and performed both before and after a week of aerobic exercise training in subjects with T2D. Following the MMTT in the Post condition, 30 minutes of treadmill walking plus one additional day of exercise was done to maintain the training adaptations through the end of the study. 


\section{Control diet}

Meals for the control diet consisted of commonly consumed foods in the form of three main meals and an afternoon snack. The overall diet, as well as each individual meal, was comprised of $60 \%$ carbohydrate $(\mathrm{CHO}), 30 \%$ fat, and $10 \%$ protein. In the "Pre" condition (before the exercise training intervention), subjects were asked to leave at least 3 hours between meals and to try to eat at the same time each day. They were given recording sheets and asked to record the precise time of each meal or snack and note any variations from the study diet (e.g., if they did not finish something). In the "Post" condition (following the exercise training intervention), subjects were given a copy of their logs from the Pre condition and asked to replicate them exactly; they again recorded the exact time each meal was consumed and any variations (though variations were strongly discouraged). Thus, the control diet was exactly the same in the Pre and Post conditions, with the exception of the re-feeding protocol during the Post condition, described elsewhere.

\section{Exercise training}

Heart rates achieved during the maximal graded exercise test were used to calculate exercise intensity as a percentage of heart rate reserve (HRR), where HRR = $\left[\left(\mathrm{HR}_{\text {peak }}-\mathrm{HR}_{\text {rest }}\right) \mathrm{x} \%\right.$ intensity $]+\mathrm{HR}_{\text {rest }}$, derived from the Karvonen formula (94). Aerobic exercise was prescribed at $60 \%$ of HRR, chosen because it is considered to be at the high range of moderate intensity $(23,68)$ with previous studies demonstrating its tolerability in this relatively unfit population $(120,121,135)$.

Each daily exercise session started with of 20 minutes of brisk treadmill walking on an incline, followed immediately by 20 minutes of stationary cycling against 
resistance and then another 20 minutes of treadmill walking. Total time spent exercising was 60 minutes for each session. All exercise sessions were supervised in the Exercise Physiology fitness laboratory by trained study staff, and intensity was continuously monitored using heart rate telemetry. Seven consecutive days of exercise training were performed prior to the Post MMTT. Because the MMTT was a long testing day requiring subjects to remain supine in bed, completely sedentary, 30 minutes of treadmill walking was done immediately following the MMTT to counter the inactivity imposed by the test. Additionally, one regular exercise bout was performed the day after the MMTT study day so that any acute effects of exercise on each of the tests (MMTT and OGTT) would remain constant.

Subjects performed the majority of exercise sessions in the late afternoon, before the dinner meal, and the last exercise session before the MMTT or OGTT was completed 14-16 hours prior to the study day testing meal or glucose challenge. To minimize subject discomfort and reduce risk for injury and boredom, subjects alternated from the treadmill to the bike and back to the treadmill for the workout, as this model has previously shown to be successful in our laboratory (121). Subjects' feet were checked for sores, blisters, and abrasions prior to each workout, and blood glucose was taken using a handheld glucometer before and after each exercise session, with a small capillary blood sample obtained via finger prick. If blood glucose values were $<100 \mathrm{mg} / \mathrm{dL}$ prior to exercise, a snack (juice box and crackers) was administered, and glucose concentration was rechecked before starting the exercise session. This was incorporated into the exercise protocol because some subjects with T2D could experience hypoglycemia with exercise if blood glucose levels are low upon exercise initiation (42). To compensate for the 
energy expenditure from the exercise sessions, snacks of 350 kilocalories (kcal) were given to offset the energy deficit and prevent weight loss, as this has been done previously and has been successful in maintaining energy balance in a similar population (121). Subjects were instructed to consume the additional energy with their afternoon snack.

\section{MMTT and OGTT experimental protocols}

The MMTT employed in this study was part of another study and has been described in detail elsewhere (See "Study One"). Thus, for the purpose of the present study, only the methodology relevant to comparing outcomes of the MMTT to the OGTT is reported here. Because aforementioned data from the MMTT comprised the primary aim of the studies, the researchers decided to keep the timing of the Pre and Post MMTTs consistent rather than randomizing the order of the MMTT and OGTT.

For each testing day, subjects arrived to the laboratory following a 12 hour overnight fast and had not taken any morning medications, including those prescribed for T2D. A research nurse or trained technician placed an inline catheter into an antecubital vein for frequent blood sampling, and the arm was heated throughout the test for collection of arterialized venous blood samples. Baseline blood samples were collected prior to consumption of the meal or glucose drink. The clock was reset to zero immediately following the challenge meal or beverage, and blood samples were collected frequently over the first 30 minutes and every 30 minutes over the next four hours. Samples were placed in serum separator and EDTA tubes containing aprotinin and dipeptidyl-peptidase IV inhibitors. Before centrifugation, SST tubes were allowed to sit at room temperature for 30 minutes with EDTA tubes chilled on ice during this time. 
Samples were spun at $3500 \mathrm{rpm}$ for 15 minutes, and aliquots were stored at $-80^{\circ} \mathrm{C}$ until analysis.

The meal challenge for the MMTT consisted of a breakfast wrap with orange juice (Table 3). Subjects consumed the meal in approximately five minutes, with half of the orange juice sipped while eating the wrap and the other half consumed quickly immediately after the last bite of wrap was ingested. The precise time of meal consumption was noted during the Pre condition and replicated for the Post condition.

A $75 \mathrm{~g}$ standard glucose beverage (Azer Scientific, Morgantown, PA) was used as the glucose challenge for the OGTT. Following collection of a baseline blood sample, subjects were instructed to drink the glucose beverage within two minutes, with the exact time recorded during the Pre condition and replicated for the Post condition. 
Table 3. Composition of the MMTT meal challenge.

\begin{tabular}{lrrrrr}
\hline Food & $\begin{array}{r}\text { Serving } \\
(\mathbf{g})\end{array}$ & $\begin{array}{r}\text { CHO } \\
(\mathbf{g})\end{array}$ & $\begin{array}{r}\text { Fat } \\
(\mathbf{g})\end{array}$ & $\begin{array}{r}\text { Protein } \\
(\mathbf{g})\end{array}$ & $\begin{array}{r}\text { Energy } \\
(\mathbf{k c a l})\end{array}$ \\
\hline Flour tortilla & 45.9 & 23.4 & 3.3 & 3.7 & 140.5 \\
Egg beaters & 2.0 & 0 & 0 & 0.2 & 1.1 \\
Egg yolk (from large egg) & 7.7 & 0.2 & 1.2 & 0.7 & 14.5 \\
American cheese & 18.0 & 1.7 & 4.3 & 2.6 & 60 \\
Margarine spread & 9.0 & 0 & 4.5 & 0 & 38.6 \\
100\% orange juice, pulp free & 321.0 & 34.8 & 0 & 2.7 & 147.1 \\
TOTAL & & $\mathbf{6 0 . 1}$ & $\mathbf{1 3 . 3}$ & $\mathbf{9 . 9}$ & $\mathbf{4 0 1 . 8}$ \\
\hline
\end{tabular}




\section{Calculations}

$\boldsymbol{A} \boldsymbol{U C}$. Paired samples t-tests were used to assess baseline differences for glucose, insulin, and c-peptide for determination of whether total area under the curve (AUC) or incremental area under the curve (iAUC) should be used in analysis. Because no baseline differences were found for glucose, insulin, or c-peptide, total AUC was calculated for each of these measures using the trapezoidal method, as described previously $(26,194)$.

Percent (\%) change. The \% change in variables from Pre to Post was calculated using the following formula: $\%$ change $=[($ Post-Pre $) /$ Post $] * 100$. Negative $\%$ change values indicate a reduction in the variable with exercise training, while positive $\%$ change indicates that the variable increased with exercise training.

Matsuda ISI. The Matsuda ISI was calculated using baseline and average glucose and insulin concentrations throughout the tests as described previously (115).

$\boldsymbol{\beta}$-cell function. The ratio of the change in c-peptide concentrations to the change in glucose concentrations early in the test was used as an estimate of $\beta$-cell function using the formula, $\beta$-cell function $=\Delta \mathrm{c}$-peptide $(30-0) / \Delta$ glucose $_{(30-0)}$.

Insulin clearance. Hepatic insulin extraction, or insulin clearance, was calculated using the molar ratio of insulin to c-peptide at each time point, by the following formula: $\%$ hepatic insulin extraction $=\left[1-\left(\operatorname{insulin}_{(\mathrm{pmol} / \mathrm{L})} / \mathrm{c}-\right.\right.$ $\left.\left.\operatorname{peptide}_{(\mathrm{pmol} / \mathrm{L})}\right)\right]^{* 100}$. Individual time values were used to calculate AUC for insulin clearance.

\section{Statistical analysis}

Data were analyzed using IBM SPSS Statistical Package, version 22 (IBM Corporation, Armonk, NY). Two-way repeated measures analysis of variance (ANOVA) 
was used to determine if overall main effects existed among variables between the tests (MMTT and OGTT) and the treatments (Pre and Post exercise training). Where significant main effects were found, paired samples t-tests were used to detect test and treatment differences for individual variables. Significance was accepted at $\mathrm{p}<0.05$. Percent change in variables from Pre to Post exercise training for the MMTT and the OGTT were compared using paired samples t-tests.

\section{Results}

Six subjects completed all testing protocols, and baseline subject characteristics are shown in Table 4. Adherence to exercise training was $100 \%$, with all subjects completing every exercise session. There was no change in body weight following the exercise training intervention $(\mathrm{p}>0.05)$. 
Table 4. Subject characteristics at screening $(n=6)$.

\begin{tabular}{|c|c|}
\hline Variable & Mean \pm SEM \\
\hline Age (years) & $58 \pm 3$ \\
\hline $\operatorname{Sex}(\mathrm{F} / \mathrm{M})$ & $4 / 2$ \\
\hline Body Weight (kg) & $97.7 \pm 9.5$ \\
\hline Body Mass Index $\left(\mathrm{kg} / \mathrm{m}^{2}\right)$ & $34.5 \pm 2.6$ \\
\hline Body Fat (\%) & $36.8 \pm 2.0$ \\
\hline Total Cholesterol (mg/dl) & $149 \pm 7$ \\
\hline LDL-C (mg/dl) & $79 \pm 8$ \\
\hline HDL-C (mg/dl) & $43 \pm 4$ \\
\hline Triglycerides (mg/dl) & $136 \pm 24$ \\
\hline Hemoglobin A1c (\%) & $6.9 \pm 0.2$ \\
\hline Fasting blood glucose (mg/dl) & $131 \pm 16$ \\
\hline Duration of diabetes (years) & $5 \pm 2$ \\
\hline Relative $\mathrm{VO}_{2 \text { peak }}\left(\mathrm{ml} \cdot \mathrm{kg}^{-1} \cdot \mathrm{min}^{-1}\right)$ & $22.1 \pm 1.2$ \\
\hline
\end{tabular}




\section{Glucose, insulin, and c-peptide}

The two-way repeated measures ANOVA for measures of postprandial glycemic control (glucose, insulin, and c-peptide AUC) revealed an overall main effect for treatment (Pre vs. Post exercise training; $p<0.01$ ) and an overall main effect for test type (MMTT vs. OGTT; $p<0.05)$. There was no significant interaction effect for treatment by test type. The results of post-hoc paired samples t-tests for individual variables are thus reported where indicated. Percent change between Pre and Post exercise training also is described. All data are reported as mean \pm standard error of the mean (SEM).

Glucose. Glucose responses to the MMTT and the OGTT for both Pre and Post exercise training are shown in Figure 11). Comparisons indicated no difference in glucose AUC by treatment (Pre and Post exercise training; $p>0.05$ ), but glucose AUC was lower for the MMTT than for the OGTT (MMTT: $31784 \pm 2434 \mathrm{mg} \cdot \mathrm{min} / \mathrm{dL}$ vs.

OGTT: $42463 \pm 4456 \mathrm{mg} \cdot \mathrm{min} / \mathrm{dL} ; \mathrm{p}<0.05)$. The change in glucose AUC with exercise training was $-5.1 \pm 3.2 \%$ for the MMTT and $-3.1 \pm 5.8 \%$ for the OGTT and was not significantly different between the tests $(\mathrm{p}>0.05)$. 


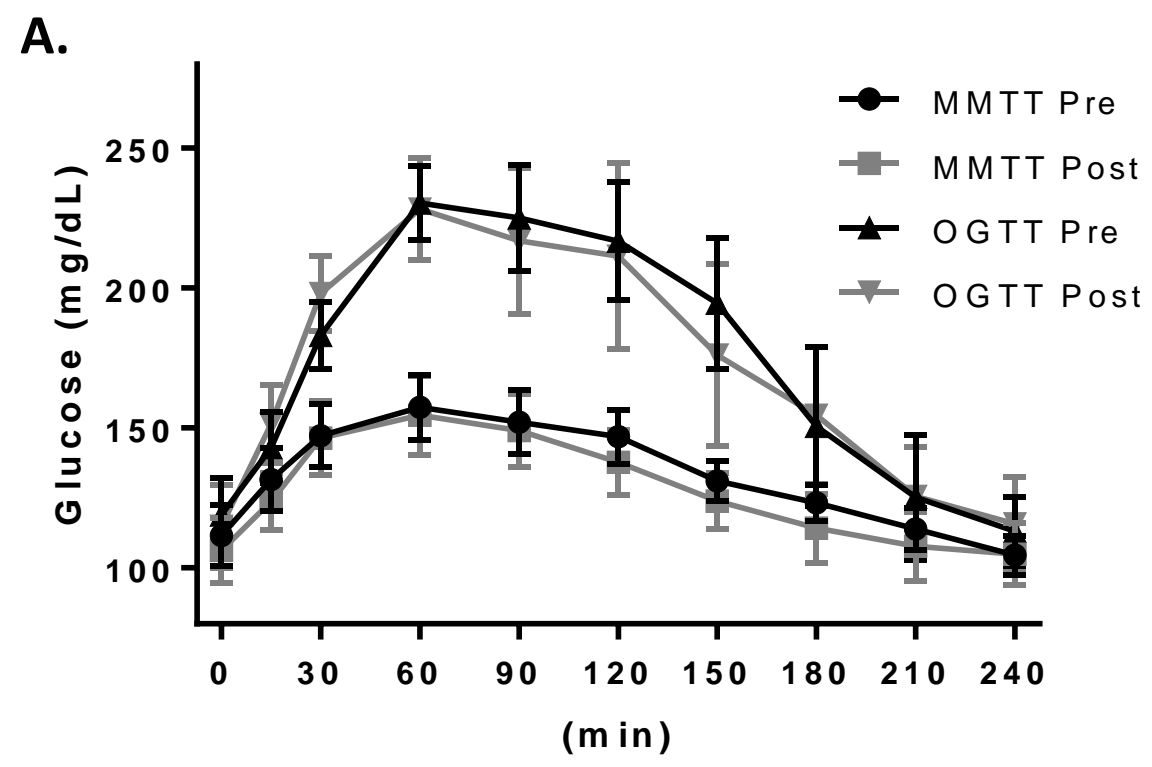

B.

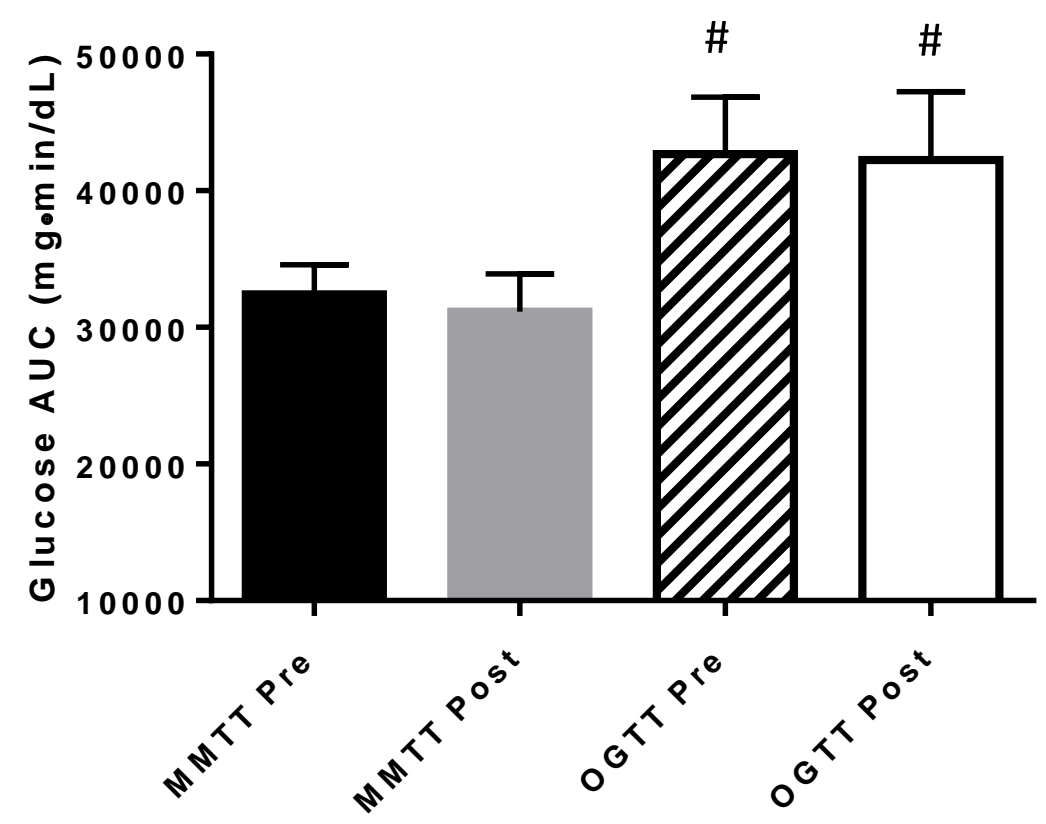

FIGURE 11 - Glucose responses to MMTTs and OGTTs performed before (Pre) and after (Post) one week of aerobic exercise training in subjects with T2D. A.) Blood glucose concentrations over time. B.) Glucose AUC. Data are expressed as mean \pm SEM. * Pre vs. Post, $\mathrm{p}<0.05$; \# MMTT vs. OGTT, $\mathrm{p}<0.05$. 
Insulin. Insulin AUC was significantly lower after exercise training compared to before (Pre: $18059 \pm 1757 \mathrm{mg} \cdot \mathrm{min} / \mathrm{dL}$ vs. Post: $13316 \pm 1126 \mathrm{mg} \cdot \mathrm{min} / \mathrm{dL} ; \mathrm{p}<0.01)$ and was also different between the MMTT and the OGTT tests, with higher insulin AUC observed for the OGTT (MMTT: $12031 \pm 920 \mu \mathrm{IU} \cdot \mathrm{min} / \mathrm{mL}$ vs. OGTT: $19345 \pm 2036$ $\mu \mathrm{IU} \cdot \mathrm{min} / \mathrm{mL} ; \mathrm{p}<0.01)$. Insulin AUC was significantly lower Post vs. Pre for both the MMTT (Pre: $13233 \pm 1120 \mu \mathrm{IU} \cdot \mathrm{min} / \mathrm{mL}$ vs Post: $10828 \pm 861 \mu \mathrm{IU} \cdot \mathrm{min} / \mathrm{mL} ; \mathrm{p}<0.05$ ) and for the OGTT (Pre: $22886 \pm 2716 \mu \mathrm{IU} \cdot \mathrm{min} / \mathrm{mL}$ vs. Post: $15804 \pm 1740 \mu \mathrm{IU} \cdot \mathrm{min} / \mathrm{mL}$; $\mathrm{p}<0.05)$. These responses are shown in Figure 12. Exercise training resulted in a change of $-23.0 \pm 8.3 \%$ in circulating insulin for the MMTT and $-46.0 \pm 14.4 \%$ for the OGTT, but this change was not significantly different between the two tests $(\mathrm{p}>0.05)$.

C-peptide. The responses for c-peptide to the glucose and meal challenges are shown in Figure 13. C-peptide AUC was significantly lower Post compared to Pre exercise training (Pre: $2439 \pm 167 \mathrm{ng} \cdot \mathrm{min} / \mathrm{mL}$ vs. Post: $2069 \pm 191 \mathrm{ng} \cdot \mathrm{min} / \mathrm{mL} ; \mathrm{p}<0.01$ ). However, there was no difference between the MMTT and the OGTT tests $(\mathrm{p}>0.05)$. Cpeptide AUC was significantly lower after exercise training than before training for both the MMTT (Pre: $2082 \pm 205 \mathrm{ng} \cdot \mathrm{min} / \mathrm{mL}$ vs. Post: $1774 \pm 163 \mathrm{ng} \cdot \mathrm{min} / \mathrm{mL} ; \mathrm{p}<0.05$ ) and for the OGTT (Pre: $2795 \pm 338 \mathrm{ng} \cdot \mathrm{min} / \mathrm{mL}$ vs. Post: $2364 \pm 344 \mathrm{ng} \cdot \mathrm{min} / \mathrm{mL} ; \mathrm{p}<0.05$ ). Exercise training resulted in a similar $-17.5 \pm 4.2 \%$ change in c-peptide concentration in the blood for the MMTT and $-21.3 \pm 7.7 \%$ for the OGTT $(\mathrm{p}>0.05)$. 
A.

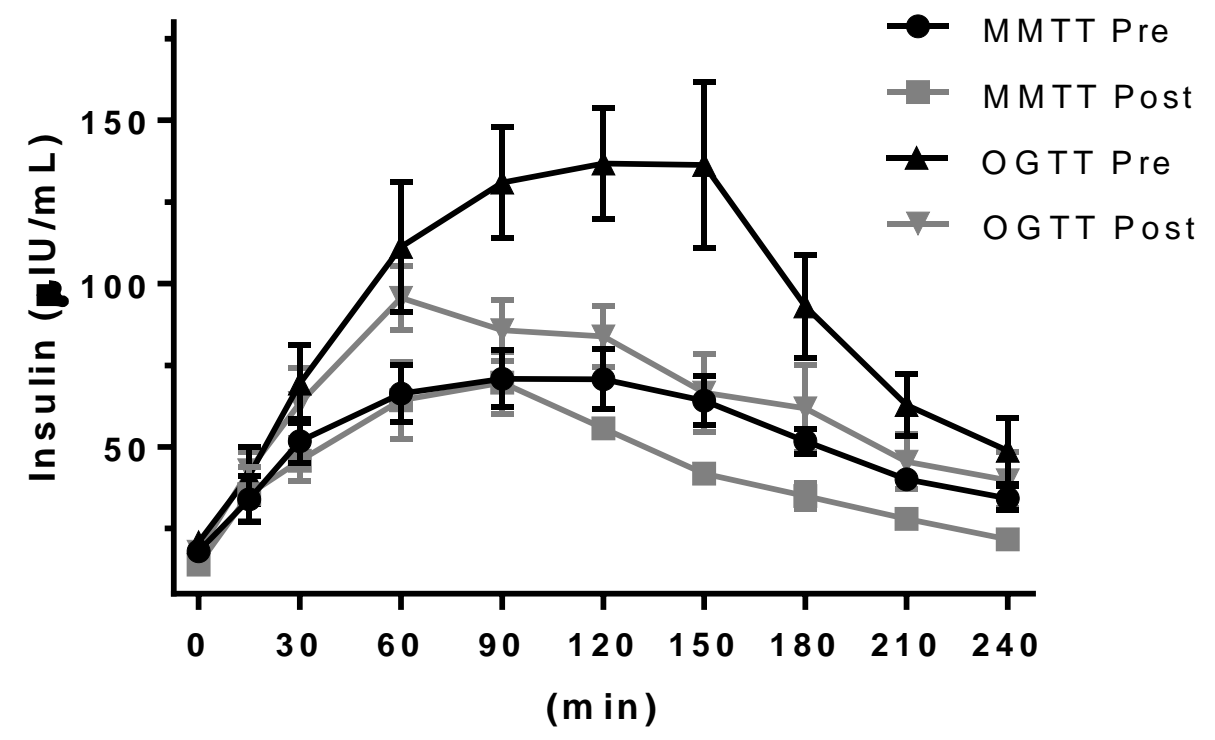

B.
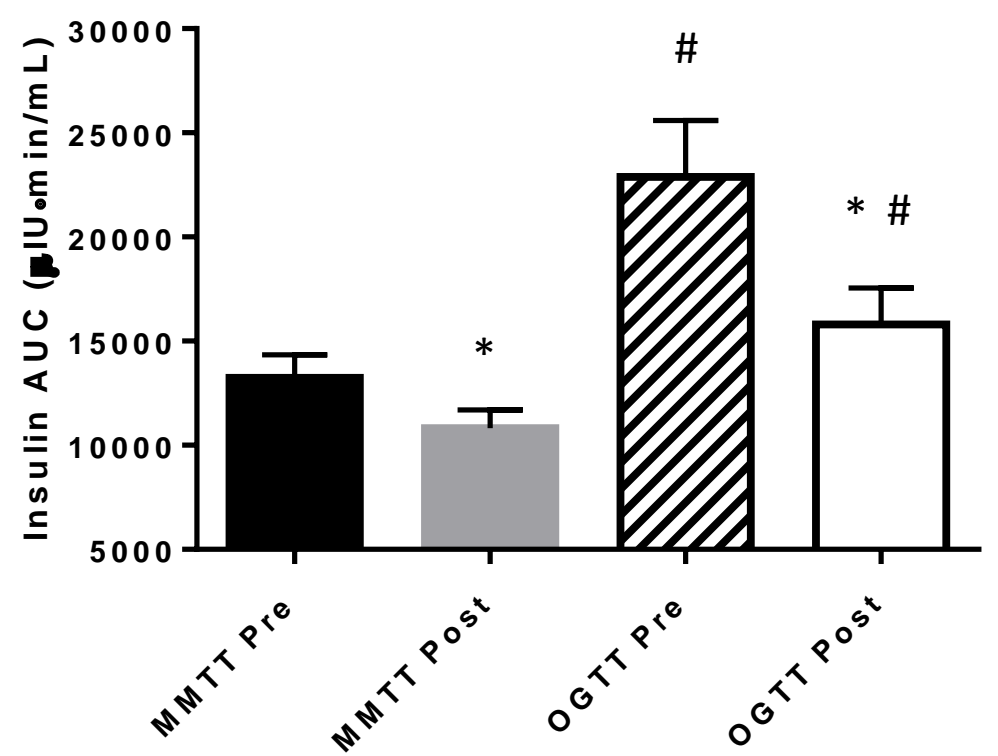

FIGURE 12- Insulin responses to MMTTs and OGTTs performed before (Pre) and after (Post) one week of aerobic exercise training in subjects with T2D. A.) Insulin concentrations over time. B.) AUC for the postprandial insulin response. Data are expressed as mean \pm SEM. * Pre vs. Post, $p<0.05$; \# MMTT vs. OGTT, $p<0.05$. 


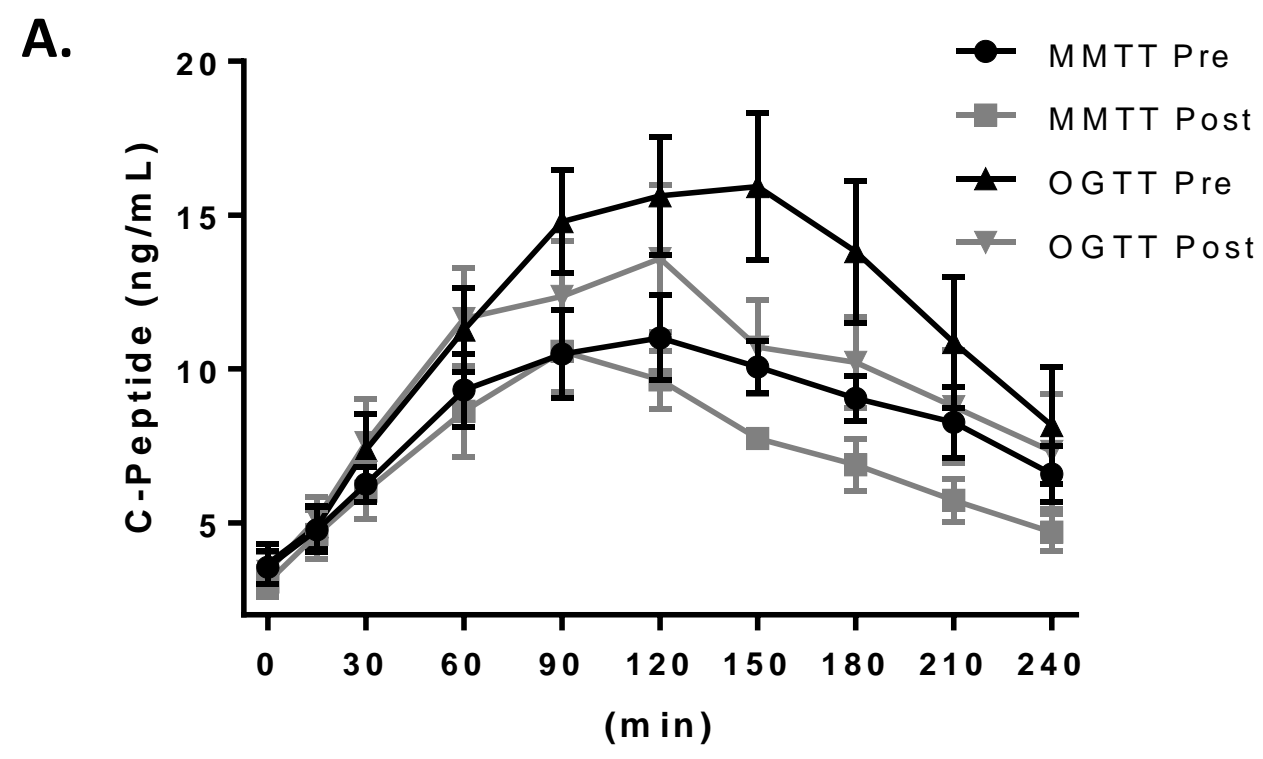

B.
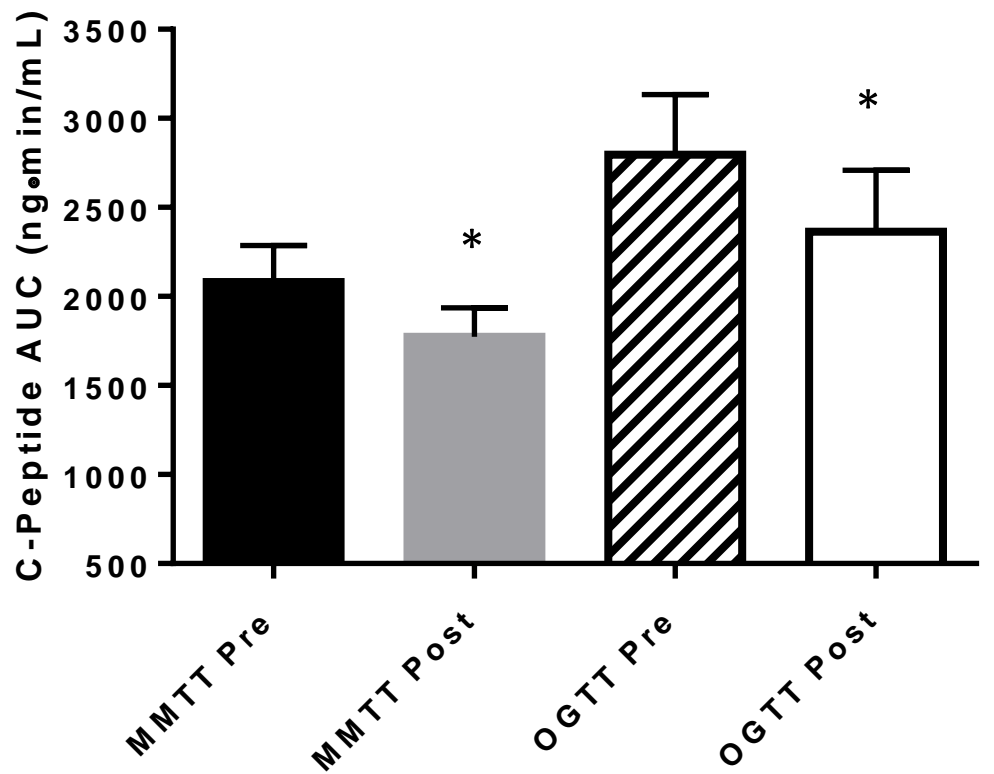

FIGURE 13 - C-peptide responses to MMTTs and OGTTs performed before (Pre) and after (Post) one week of aerobic exercise training in subjects with T2D. A.) Cpeptide concentrations over time. B.) AUC for the postprandial c-peptide response. Data are expressed as mean \pm SEM. * Pre vs. Post, $p<0.05$; \# MMTT vs. OGTT, p $<0.05$. 
Insulin clearance. Because of the observed differences in insulin responses between the MMTT and the OGTT with no difference in c-peptide between the tests, insulin clearance was assessed and expressed as AUC for \% hepatic insulin extraction (insulin clearance), and is shown in Figure 14. There was a significant main effect for insulin clearance AUC for treatment, with clearance higher Pre compared to Post exercise training (Pre: $3532 \pm 482 \% \cdot \min$ vs. Post: $3114 \pm 386 \% \cdot \min ; \mathrm{p}<0.05$ ). There were no significant main effects for test type or for interaction of treatment and test. However, paired samples t-tests for treatment revealed that the Pre vs. Post differences were driven by differences in the OGTT (Pre: $4040 \pm 857 \% \cdot \min$ vs. Post: $3422 \pm 697 \% \cdot \min ; \mathrm{p}<$ $0.05)$ that were not present in the MMTT ( $p>0.05)$. The change in \% hepatic insulin extraction AUC was $-6.2 \pm 5.6 \%$ for the MMTT and $-19.5 \pm 7.8 \%$ for the OGTT; $p$ > $0.05)$. 
A.

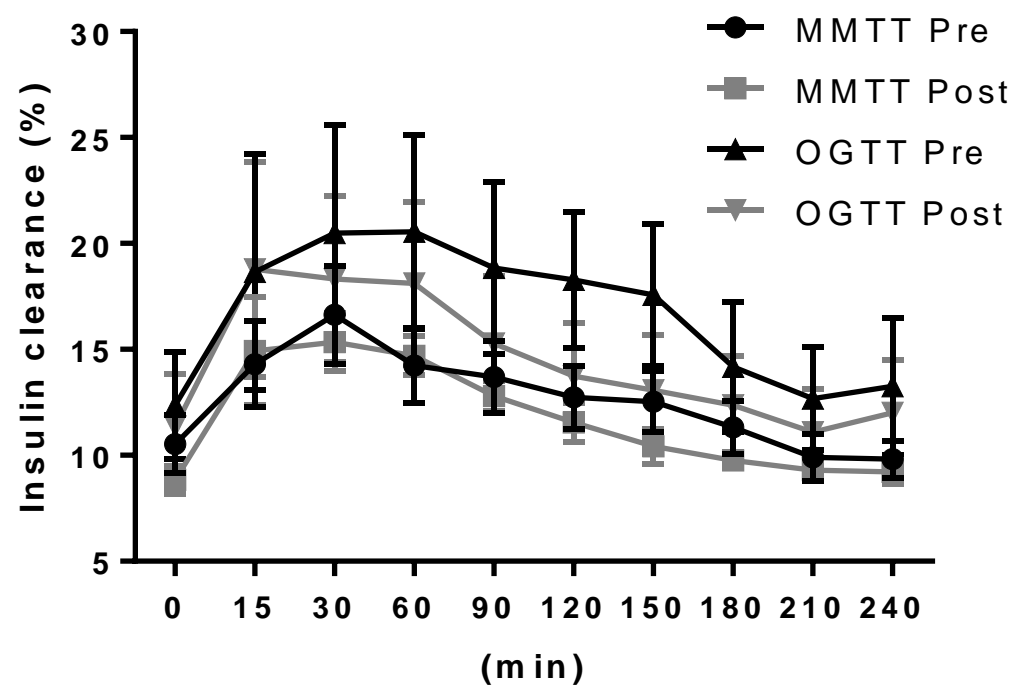

B.

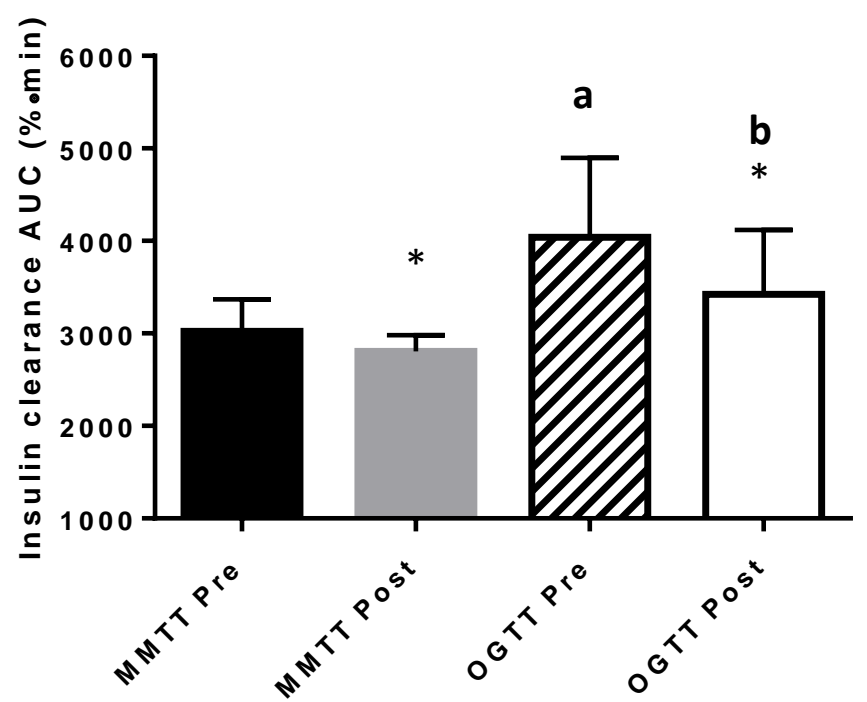

FIGURE 14 - Postprandial insulin clearance following MMTTs and OGTTs performed before (Pre) and after (Post) one week of aerobic exercise training in subjects with T2D. A.) \% insulin clearance over time. B.) AUC for the 4 hour postprandial insulin clearance. Data are expressed as mean \pm SEM. * Pre vs. Post main effect, $\mathrm{p}<0.05$; \# MMTT vs. OGTT main effect, $\mathrm{p}<0.05$. Differences between letters denote post-hoc Pre vs. Post differences within test type. 


\section{Matsuda ISI and $\beta$-cell function}

The two-way repeated measures ANOVA revealed an overall main effect for both test type $(\mathrm{p}<0.01)$ and treatment $(\mathrm{p}<0.01)$ for analysis of calculated measures of $\beta$-cell function and the Matsuda ISI, but there was no significant interaction effect for test type by treatment.

Univariate analysis and post-hoc tests indicated that the Matsuda ISI was significantly higher with the MMTT than with the OGTT (MMTT: $3.51 \pm 0.52$ a.u. vs. OGTT: $2.09 \pm 0.25$ a.u.; $p<0.01)$ and was also different between the Pre and Post conditions, with a higher Matsuda ISI after exercise training indicative of greater insulin sensitivity (Pre: $2.34 \pm 0.24$ a.u. vs. Post: $3.27 \pm 0.53$ a.u.; $p<0.05$ ), as illustrated in

Figure 15. Paired samples t-tests indicated that these Pre vs. Post effects were significant both for the MMTT (Pre: $2.90 \pm 0.30$ a.u. vs. Post: $4.12 \pm 0.75$ a.u.; $p<0.05$ ) and for the OGTT (Pre: $1.77 \pm 0.21$ a.u. vs. Post: $2.41 \pm 0.33$ a.u.), with greater insulin sensitivity after exercise training seen for both tests. The MMTT vs. OGTT differences were due to differences for both Pre (MMTT: $2.90 \pm 0.30$ a.u. vs. OGTT: $1.77 \pm 0.21$ a.u.; $p<0.05$ ) and Post conditions (MMTT: $4.12 \pm 0.75$ a.u. vs. OGTT: $2.41 \pm 0.33$ a.u.; $p<0.05$ ). The change in the Matsuda ISI from Pre to Post was a similar $24.3 \pm 6.8 \%$ for the MMTT and $23.5 \pm 7.7 \%$ for the OGTT $(\mathrm{p}>0.05)$

There were no differences in $\beta$-cell function between the two test types $(p>0.05)$ or between treatments $(\mathrm{p}>0.05)$. 


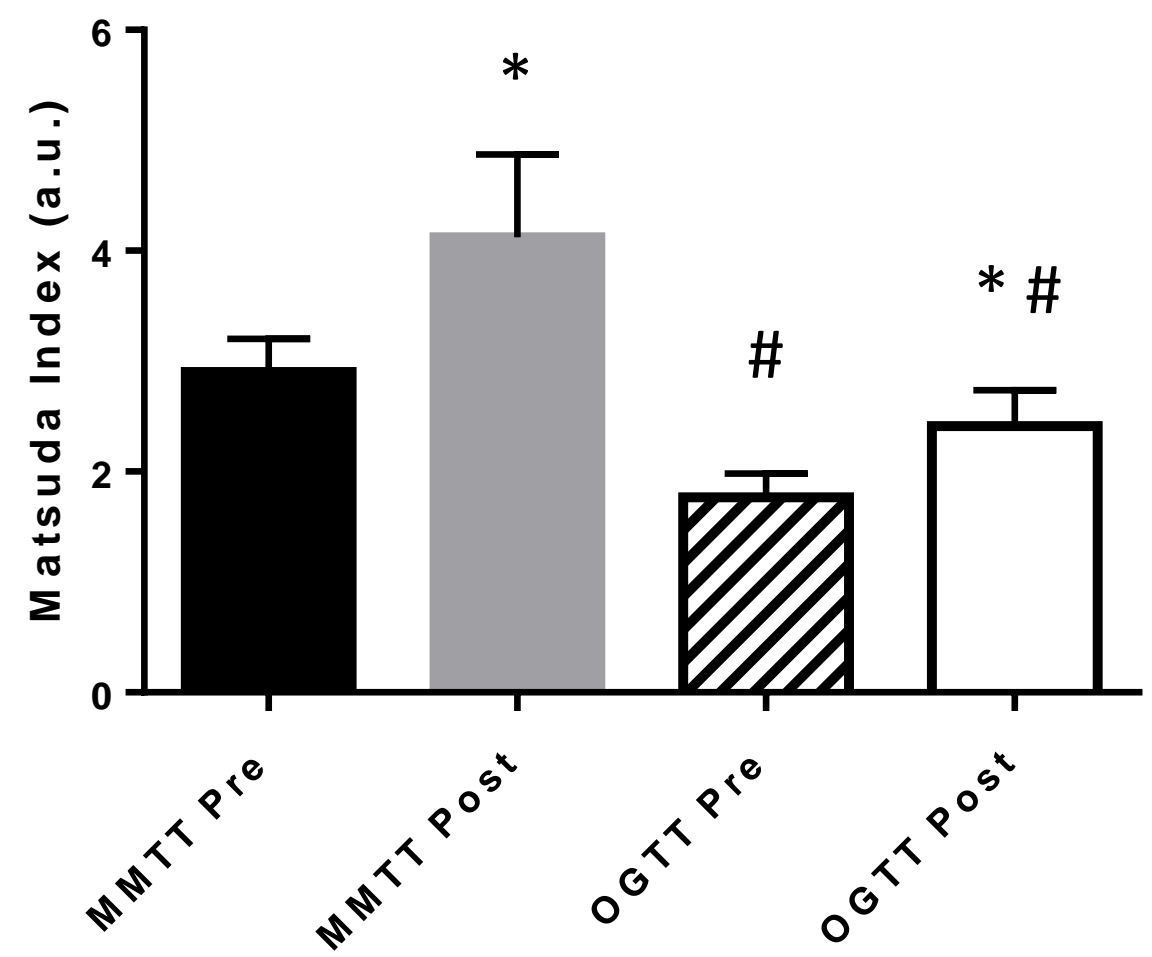

FIGURE 15 - Differences in the calculated Matsuda insulin sensitivity index among mixed meal tolerance tests (MMTT) and oral glucose tolerance tests (OGTT) performed before (Pre) and after (Post) one week of aerobic exercise training in subjects with T2D. Data are expressed as mean \pm SEM. * Pre vs. Post, $p<0.05$; \# MMTT vs. OGTT, $\mathrm{p}<0.05$. 


\section{Discussion}

The primary novel finding of this study was that both the MMTT and the OGTT were similar in their abilities to detect changes in factors underlying postprandial glycemic control, but neither test type found a change in PPG following the short-term aerobic exercise training intervention. Exercise training reduced postprandial insulin and postprandial c-peptide similarly for both the MMTT and the OGTT, and there was no difference in PPG following exercise training for either of the tests. Additionally, insulin sensitivity, as calculated by the Matsuda ISI, was improved similarly between the two tests following the intervention, and there were no changes in $\beta$-cell function with one week of exercise training.

Compared to the OGTT, the MMTT elicited a lesser overall postprandial response for glucose and insulin. Yet, the change from Pre to Post exercise training was not significantly different, and subjects responded similarly within tests following the shortterm exercise training intervention.

Overall, the way subjects responded before and after exercise training was not different for the two test types. It should be noted that there was a large degree of variability among subject responses, and thus the lack of significance for an interaction effect or difference in \% change with exercise training between the two tests should be interpreted with caution. This is particularly true with the small sample sizes used in this study. It is possible that having more subjects would increase the likelihood of an interaction effect, and future work should examine this. Even so, the overall conclusion that the tests, in general, produced similar results, is fairly robust. Many Pre vs. Post differences were found in measures of postprandial glycemic control, even with the 
variability seen among subjects, and these responses were similar between the two test types.

Notably, the postprandial insulin response was significantly greater for the OGTT than for the MMTT, but the postprandial c-peptide response was not different. C-peptide is cleaved from pro-insulin upon release from the pancreatic $\beta$-cell before insulin is partially cleared by the liver and generally is correlated with circulating insulin concentrations $(79,155)$. Because c-peptide reflects insulin secretion, it is unusual that the c-peptide response did not directly mirror the insulin response. Therefore, we examined insulin clearance to determine if a change in hepatic insulin extraction might be responsible for this difference. Although we found a difference in insulin clearance between the Pre and Post treatment conditions, it did not explain the differential responses between insulin and c-peptide. Insulin clearance was higher before exercise training than after training but not different between the OGTT and the MMTT tests. However, the Pre vs. Post difference in insulin clearance appears to be driven by a significant decrease in insulin clearance with the OGTT that was not present with the MMTT. Still, this does not explain why c-peptide concentrations were not different between the MMTT and OGTT tests while insulin concentrations were.

In this population of subjects with T2D, glucose and insulin responses were greater with the OGTT test than with the MMTT test. This is in agreement with results from Wolever et al. (193) who showed that a $75 \mathrm{~g}$ OGTT resulted in greater glucose and insulin levels when compared to a MMTT in subjects of varying levels of glucose tolerance. This group attributed the difference to a higher total carbohydrate load in the glucose beverage than in the meal ( $75 \mathrm{~g}$ in the beverage versus $50 \mathrm{~g}$ in the meal), as well 
as a higher glycemic index in the glucose beverage. However, our results are in contrast to the results observed by Traub et al. in older non-diabetic women (181). Traub and colleagues found that 2 hour glucose following a $75 \mathrm{~g}$ OGTT was lower than following two different types of mixed meal tests. One of the mixed meal tests used in the study by Traub was matched for carbohydrate (75 g) and thus had significantly more total energy than the glucose beverage $(600 \mathrm{kcal})$. However, the other mixed meal test, termed the "muffin test," contained less carbohydrate $(56 \mathrm{~g})$ and only $410 \mathrm{kcal}$ of total energy. Yet, both meal tests elicited higher 2 hour blood glucose concentrations compared to the OGTT.

Potential explanations for the discrepancy among studies include the difference in subject populations, testing methods, and differences in digestion and absorption of pure glucose and meals of mixed macronutrient composition. Traub et al. (181) examined nonobese, non-diabetic subjects and only showed 2 hour glucose concentrations rather than glucose responses over a postprandial time course. It is possible that in metabolically healthy subjects, the higher glycemic index of pure glucose would cause an earlier peak than glucose polymers in a mixed meal and would normalize within 2 hours, whereas starch absorption occurs later and could be the cause of the higher 2 hour glucose levels. This phenomenon, related to the glycemic index, has been previously described by Jenkins, Wolever, and colleagues; the glycemic index of foods and the overall glycemic load of meals affect the rate of glucose absorption, with beverages and meals containing high glycemic index foods (such as in the OGTT) are absorbed more quickly than mixed macronutrient meals which have a lower glycemic load $(86,87,194)$. 
Additionally, compared to normal healthy weight and non-diabetic subjects, obese individuals and those with T2D have been purported to have increased glucose absorption in the proximal intestine contributing to hyperglycemia, attributed to increased levels of the intestinal glucose transporters (SGLT1, GLUT2, and GLUT5) (57, 132, 197). Further, previous work has shown that there can be marked differences in gastric emptying with diabetes (both type 1 and type 2), with between 30 and 50\% of diabetic patients exhibiting delayed gastric emptying (78). Thus, it is possible that differences in absorption for individuals with T2D could contribute to differences among studies.

Because both the present study and the study by Wolever and colleagues examined glucose at multiple time points following meal or glucose consumption, these studies would better capture the true postprandial response than the study by Traub et al. (181). Further, the present study examined only people with T2D and the study by Wolever et al. (193) included subjects across the glucose tolerance spectrum, including those with T2D. And, as demonstrated in previous work, people with T2D have altered glucose metabolism including delayed uptake and reduced inhibition of endogenous glucose production $(48,61)$. Thus, it is possible that differences between the studies may also be attributed to differences in glucose metabolism between healthy subjects, diabetic subjects, and subjects with varying levels of glucose tolerance.

\section{Limitations}

There are limitations to the present study which should be recognized. First, as mentioned previously, the order of the MMTT and OGTT tests was not randomized, and the OGTT always occurred two days after the MMTT. Randomizing these tests would 
undoubtedly have made for a stronger design for this study.Nevertheless, because the MMTT was part of a larger study in which it was critical not to vary its timing, the researchers decided that it was more important to maintain consistency for the primary aim of the dissertation and accept the limitation for the present study. Had our hypotheses held true, the non-randomized timing of the tests would not have been a major limitation. This is because, based on outcomes from previous work in which the OGTT did not show changes in glucose concentrations following seven days of exercise training (120), it was hypothesized that the OGTT data in this study also would not be significantly changed but that the glucose responses to the MMTT, a more physiologic test that was not expected to inhibit hepatic glucose output to the same degree as with the OGTT, would change. Thus, if this was found to be true, putting the OGTT after the MMTT with one additional day of exercise separating the tests would still allow us to robustly conclude that our results indicated that the MMTT is a more sensitive test to detect changes in PPG following short-term exercise training. However, the data did not support this hypothesis. While the OGTT responses were similar to those observed with the MMTT with regard to Pre vs. Post exercise training, the OGTT produced greater glucose responses overall. Thus, it could be argued that having the additional exercise bout prior to the OGTT confounds our interpretation. It is possible that the extra day of exercise provided an added stimulus for the OGTT that was not present for the MMTT. Even so, it is unlikely that one additional day of exercise elicited a stimulus strong enough to produce changes in the OGTT that otherwise would not have existed. Nonetheless, future studies comparing the MMTT and the OGTT should randomize the order of the two tests. 
Another limitation to the present study is the sample size. Based on initial power analyses for multiple variables, our goal was to have at least 10 subjects complete all testing protocols. Yet subject recruitment proved more difficult than anticipated, and most potential subjects were not willing to commit to the large number of visits, time involvement, and strict controlled feeding and monitoring required by this study. Thus, future efforts should be made to reduce the number of visits required by subjects as well as to reduce the time burden of self-monitoring outside the lab. This is extremely difficult with controlled feeding and exercise training studies, as monitoring is important to ensure compliance and reproducibility, and each study visit served a distinct purpose. Potential recommendations to combat this for future studies would be to reduce the controlled feeding portion and to allow the subjects to perform one exercise bout outside of the laboratory (using an activity monitor as insurance the session was completed) for shortterm exercise training protocols. Additionally, care should be taken to ensure that only minimal work must be done by the subjects themselves. The latter is also important in attaining adherence to all protocols, as many subjects were observed to have difficulty remembering what to do, even with the researcher discussing instructions with the subject as well as providing written instructions.

Another limitation of this study is the difference in the carbohydrate load between the MMTT and the OGTT. This is a problem that presents in every study using these tests, and there is currently no consensus as to the best way to match energy when comparing results between the tests. Some studies $(148,181)$ have matched based only on carbohydrate content (e.g., matching carbohydrate in the MMTT to the 75 g provided in the OGTT). This is not a perfect design, as carbohydrates comprise only $\sim 60 \%$ of the 
total energy for the MMTT, and with the addition of $10 \%$ protein and $30 \%$ fat, the MMTT challenge would be significantly greater in total energy content than the OGTT challenge. Other studies $(181,193)$ have more closely approximated total energy, but this comes at the expense of a smaller carbohydrate load in the MMTT challenge. Traub et al. (181) approached this problem by comparing blood glucose responses to three different types of tests: 1) a standard OGTT (75 g glucose; $300 \mathrm{kcal}), 2$ ) a MMTT consisting of a $600 \mathrm{kcal}$ shake [50\% of energy from carbohydrate (75g glucose), 30\% energy from fat, and 20\% energy from protein], and 3) a "muffin test" consisting of a commercially prepared breakfast muffin (average $410 \mathrm{kcal} ; 56 \mathrm{~g}$ carbohydrate, $18 \mathrm{~g}$ fat, $6 \mathrm{~g}$ protein) plus either coffee or tea. Traub found that blood glucose concentration after 2 hours was lower in the OGTT condition compared to either meal mixed meal test (MMTT or muffin test). Thus, because there is still no standardized comparison for the MMTT and OGTT, we decided to utilize a $400 \mathrm{kcal}$ meal with $60 \%$ energy from carbohydrate most closely resembling Traub's muffin test, as this provided a middle ground between the extreme options discussed previously; it contained more total energy but less carbohydrate than the OGTT. Further, the meal composition was chosen to mimic common breakfast consumption patterns in the United States.

\section{Conclusion}

In summary, glucose responses to the MMTT were lower than to the OGTT, but the exercise training intervention did not lower postprandial glucose in this population of T2D subjects, and this result was not dependent on the type of test used to assess PPG. The postprandial insulin response was greater with the OGTT than with the MMTT and 
was also lower following exercise training compared to before exercise training. Cpeptide responses did not vary between the two test types but, as expected, were lower after exercise training compared to before training. A major finding of the present study is that the way subjects responded to the exercise training intervention was not dependent on the type of test used to assess glucose. Although there are limitations discussed which warrant interpreting this finding with some degree of caution, this is a novel observation and may have important implications for the use of mixed meal tests in current clinical research and future screening and diagnostic testing. 


\section{References}

1. American College of Sports Medicine. ACSM's Guidelines for Exercise Testing and Prescription. 9th ed.: Lippincott Williams \& Wilkins; 2013.

2. American Diabetes Association. Diagnosis and classification of diabetes mellitus. Diabetes Care. 2014;37 Suppl 1:S81-90.

3. American Diabetes Association. Standards of medical care in diabetes--2014. Diabetes Care. 2014;37 Suppl 1:S14-80.

4. Boulay MR, Simoneau JA, Lortie G, Bouchard C. Monitoring high-intensity endurance exercise with heart rate and thresholds. Medicine and Science in Sports and Exercise. 1997;29(1):125-32.

5. Cavalot F, Pagliarino A, Valle M et al. Postprandial blood glucose predicts cardiovascular events and all-cause mortality in type 2 diabetes in a 14-year follow-up: lessons from the San Luigi Gonzaga Diabetes Study. Diabetes Care. 2011;34(10):2237-43.

6. Cavalot F, Petrelli A, Traversa M et al. Postprandial blood glucose is a stronger predictor of cardiovascular events than fasting blood glucose in type 2 diabetes mellitus, particularly in women: lessons from the San Luigi Gonzaga Diabetes Study. The Journal of Clinical Endocrinology and Metabolism. 2006;91(3):813-9.

7. Centers for Disease Control and Prevention. National Diabetes Statistics Report. 2014.

8. Ceriello A, Esposito K, Piconi L et al. Oscillating glucose is more deleterious to endothelial function and oxidative stress than mean glucose in normal and type 2 diabetic patients. Diabetes. 2008;57(5):1349-54. 
9. Ceriello A, Hanefeld M, Leiter L et al. Postprandial glucose regulation and diabetic complications. Archives of Internal Medicine. 2004;164(19):2090-5.

10. DeFronzo RA, Bonadonna RC, Ferrannini E. Pathogenesis of NIDDM. A balanced overview. Diabetes Care. 1992;15(3):318-68.

11. Dyer J, Wood IS, Palejwala A, Ellis A, Shirazi-Beechey SP. Expression of monosaccharide transporters in intestine of diabetic humans. American Journal of Physiology. Gastrointestinal and Liver Physiology. 2002;282(2):G241-8.

12. Ferrannini E, Simonson DC, Katz LD et al. The disposal of an oral glucose load in patients with non-insulin-dependent diabetes. Metabolism: Clinical and Experimental. 1988;37(1):79-85.

13. Garber CE, Blissmer B, Deschenes MR et al. American College of Sports Medicine position stand. Quantity and quality of exercise for developing and maintaining cardiorespiratory, musculoskeletal, and neuromotor fitness in apparently healthy adults: guidance for prescribing exercise. Medicine and Science in Sports and Exercise. 2011;43(7):1334-59.

14. Horowitz M, O'Donovan D, Jones KL, Feinle C, Rayner CK, Samsom M. Gastric emptying in diabetes: clinical significance and treatment. Diabetic Medicine: $a$ Journal of the British Diabetic Association. 2002;19(3):177-94.

15. Horwitz DL, Starr JI, Mako ME, Blackard WG, Rubenstein AH. Proinsulin, insulin, and C-peptide concentrations in human portal and peripheral blood. The Journal of Clinical Investigation. 1975;55(6):1278-83.

16. Jenkins DJ, Ghafari H, Wolever TM et al. Relationship between rate of digestion of foods and post-prandial glycaemia. Diabetologia. 1982;22(6):450-5. 
17. Jenkins DJ, Wolever TM, Taylor RH et al. Glycemic index of foods: a physiological basis for carbohydrate exchange. The American Journal of Clinical Nutrition. 1981;34(3):362-6.

18. Karvonen J, Vuorimaa T. Heart rate and exercise intensity during sports activities. Practical application. Sports Medicine. 1988;5(5):303-11.

19. Matsuda M, DeFronzo RA. Insulin sensitivity indices obtained from oral glucose tolerance testing: comparison with the euglycemic insulin clamp. Diabetes Care. 1999;22(9):1462-70.

20. McDonald GW, Burnham CE, Lewis WF. Reproducibility of glucose tolerance in 101 nondiabetic women. Public Health Reports. 1969;84(4):353-7.

21. McDonald GW, Fisher GF, Burnham C. Reproducibility of the Oral Glucose Tolerance Test. Diabetes. 1965;14:473-80.

22. Mikus CR, Fairfax ST, Libla JL et al. Seven days of aerobic exercise training improves conduit artery blood flow following glucose ingestion in patients with type 2 diabetes. Journal of Applied Physiology. 2011;111(3):657-64.

23. Mikus CR, Oberlin DJ, Libla J, Boyle LJ, Thyfault JP. Glycaemic control is improved by 7 days of aerobic exercise training in patients with type 2 diabetes. Diabetologia. 2012;55(5):1417-23.

24. Mooy JM, Grootenhuis PA, de Vries H et al. Intra-individual variation of glucose, specific insulin and proinsulin concentrations measured by two oral glucose tolerance tests in a general Caucasian population: the Hoorn Study. Diabetologia. 1996;39(3):298-305. 
25. Nguyen NQ, Debreceni TL, Bambrick JE et al. Accelerated intestinal glucose absorption in morbidly obese humans: relationship to glucose transporters, incretin hormones, and glycemia. The Journal of Clinical Endocrinology and Metabolism. 2015;100(3):968-76.

26. Oberlin DJ, Mikus CR, Kearney ML et al. One bout of exercise alters free-living postprandial glycemia in type 2 diabetes. Medicine and Science in Sports and Exercise. 2014;46(2):232-8.

27. Rijkelijkhuizen JM, Girman CJ, Mari A et al. Classical and model-based estimates of beta-cell function during a mixed meal vs. an OGTT in a populationbased cohort. Diabetes Research and Clinical Practice. 2009;83(2):280-8.

28. Rubenstein AH, Block MB, Starr J, Melani F, Steiner DF. Proinsulin and Cpeptide in blood. Diabetes. 1972;21(2 Suppl):661-72.

29. Sorkin JD, Muller DC, Fleg JL, Andres R. The relation of fasting and 2-h postchallenge plasma glucose concentrations to mortality: data from the Baltimore Longitudinal Study of Aging with a critical review of the literature. Diabetes Care. 2005;28(11):2626-32.

30. Temelkova-Kurktschiev TS, Koehler C, Henkel E, Leonhardt W, Fuecker K, Hanefeld M. Postchallenge plasma glucose and glycemic spikes are more strongly associated with atherosclerosis than fasting glucose or HbA1c level. Diabetes Care. 2000;23(12):1830-4.

31. Traub ML, Jain A, Maslow BS et al. The "muffin test"--an alternative to the oral glucose tolerance test for detecting impaired glucose tolerance. Menopause. 2012;19(1):62-6. 
32. Wolever TM, Chiasson JL, Csima A et al. Variation of postprandial plasma glucose, palatability, and symptoms associated with a standardized mixed test meal versus $75 \mathrm{~g}$ oral glucose. Diabetes Care. 1998;21(3):336-40.

33. Wolever TM, Jenkins DJ. The use of the glycemic index in predicting the blood glucose response to mixed meals. The American Journal of Clinical Nutrition. 1986;43(1):167-72.

34. Young RL, Chia B, Isaacs NJ et al. Disordered control of intestinal sweet taste receptor expression and glucose absorption in type 2 diabetes. Diabetes. 2013;62(10):3532-41. 


\section{SUMMARY AND FUTURE DIRECTIONS}

\section{Major Findings}

The primary findings of these studies were that, in subjects with T2D, one week of aerobic exercise training improved insulin sensitivity but did not improve PPG and that both the MMTT and the OGTT were similar in their abilities to detect changes in factors underlying postprandial glycemic control. Together, these studies provide evidence that enhances our understanding of the role of exercise in the treatment of T2D and how it should be assessed in the clinical setting.

Because other studies have found improvements in PPG following a week of aerobic exercise training in subjects with T2D $(109,139)$, our primary aim was to identify what tissues contribute to changes in glycemic control after short-term exercise training and identify the systemic mechanisms by which exercise lowers PPGE and improves overall glycemic control early in the course of training. We hypothesized that short-term exercise training would improve glucose disposal and decrease hepatic glucose output. Similar to others, we found that circulating insulin and c-peptide were reduced in response to a week of exercise training and that insulin sensitivity was improved $(39,108)$. However, as we saw no changes in PPG, it was not surprising that we did not observe changes in glucose Ra, EGP, or glucose Rd. Other studies also have found no changes in PPG when assessed by glucose challenge $(84,108)$, and differences could be due both to differences in study design and in subject population, including heterogeneity within T2D subjects $(43,150)$. 
Our secondary aim was to determine if a more physiologically based MMTT was a better tool for assessing changes in PPG and factors underlying postprandial glycemic control following exercise training than the standard OGTT in patients with T2D. We hypothesized that measures of postprandial glycemic control from the MMTT would be significantly improved following exercise training in diabetic subjects while the OGTT would not show improvements in glycemic control. Our primary finding was that, in contrast to what we hypothesized, the two tests were not different in their capability of detecting changes in PPG and principal factors underlying postprandial glycemic control. Our finding of equality between the tests in examining PPG following exercise training is novel. As other work has shown that the MMTT may be superior in other areas, including increased palatability, tolerability, and avoidance of reactive hypoglycemia $(25,177)$, our work should help to validate the MMTT as an alternative testing method which may still be superior to the OGTT when the whole patient is considered. However, as there is currently no standard for MMTT testing, additional work should be done to develop a more universal testing meal.

Collectively, the studies in this dissertation addressed the central hypothesis that short-term aerobic exercise training improves PPG and PPGE through improved insulin sensitivity and glucose disposal and decreased EGP, and that a mixed meal test simulating the free-living condition is a more accurate tool than the standard glucose challenge for assessing changes in glycemic control following exercise training. In general, this hypothesis was not found to be true. Because we did not see a change in PPG in these subjects following the exercise training intervention, the impact of the tissues purported to influence changes in PPG (skeletal muscle glucose disposal, liver 
glucose output, and pancreatic $\beta$-cell function) could not be determined.. Further, there were no notable differences between the type of test used to assess PPG and underlying factors (insulin, c-peptide, insulin sensitivity, and $\beta$-cell function). Based on the latter finding, we conclude that the MMTT is a valid measure of assessing postprandial glycemic control following exercise training, but it does not appear to be superior to the OGTT in detecting changes in postprandial glucose control.

\section{Perspectives and Future Directions}

The present studies raise several questions for further examination. First, we observed no changes in PPG in our subject pool, and as mentioned previously, though some other studies have observed changes in PPG in response to one week of exercise training $(109,139)$, others have not $(84,108)$. This is an important phenomenon that warrants deeper investigation. Although Solomon and colleagues have addressed the differential responses of subjects with T2D with exercise training following longer exercise training interventions (12-16 weeks), these subjects experienced changes in body composition and fitness (150). Whether or not the factors found by Solomon et al. to influence response to longer training also contribute to the heterogeneity of responses to short-term training without changes in body composition or fitness, as occurs with the one week exercise training model (139), remains unknown.

Additionally, more work needs to be done to validate the MMTT as an appropriate alternative to the OGTT in the clinical setting. While this study illustrates its efficacy in detecting changes in response to a short-term exercise training intervention when compared to the OGTT, our mixed meal is just one example of the type of meal 
that could be used. Because there are both strengths and weaknesses for using a larger meal with matched carbohydrate content compared to a smaller meal which more closely approximates the total energy of the 75 gram glucose beverage used in the standard OGTT, future studies should investigate comparisons between the postprandial responses of different sized meals and how they compare to responses observed with the OGTT.

Should money and resources not be limiting factors in study design, a greater subject pool would have enhanced the present study. Notably, examining more subjects could allow us to examine potential sex differences, differences which might be attributed to medication usage, duration of diabetes, fasting glucose levels, $\mathrm{HbAlc}$, or other physiological variables. Examining correlations between these factors and the postprandial responses could help us better understand why some subjects with T2D seem to respond more readily to exercise training than others. 


\section{EXTENDED REVIEW OF LITERATURE}

\section{Introduction}

Diabetes is a progressive disease of hyperglycemia resultant from dysfunctional insulin action and secretion and at its crux, poor glycemic control (5). Moreover, diabetes carries numerous comorbidities which may be attributed to reduced glycemic control, including neuropathy, retinopathy, nephropathy, and macrovascular problems such as coronary artery disease. Together, these morbidities limit physical function, reduce quality of life, and shorten lifespan (116). In addition to individual health problems, diabetes also induces a large financial burden both to patients and to society. The estimated economic cost of diagnosed diabetes in 2012 was $\$ 245$ billion, an amount not representing costs associated with cases of undiagnosed diabetes, which likely add vast additional expense (6). Further, a recent brief by the Health Care Cost Institute showed that per-capita health care spending in the United States on people with diabetes is steadily climbing and was more than three times greater in 2013 compared to patients without diabetes, and individual out-of-pocket costs were $\$ 1922$ for diabetic patients compared to $\$ 738$ for those without diabetes (68). While this represents both type 2 diabetes (T2D) and type 1 diabetes, T2D is by far the most prevalent, representing 90$95 \%$ of all cases (5). Exercise improves glycemic control in healthy sedentary individuals and in those with T2D and is thus a valid treatment option for this population. Moreover, lifestyle interventions including exercise and increased physical activity are an effective, fiscally sensible therapy for T2D (169). 
Not only does exercise improve glycemic control, but it also reduces the incidence of diabetes and associated diseases $(109,142)$. Yet, exercise as a first-line treatment for T2D is underutilized. This is likely partially due to a poor understanding behind the mechanisms by which exercise contributes to improved glycemic control and therefore management of T2D. It is also possible that it can be attributed in part to the inherent difficulty in employing lifestyle therapies (exercise and dietary interventions) in our current health care system. Furthermore, the ability of exercise to lower both glycosylated hemoglobin (HbA1c) and postprandial glucose, where pharmaceutical agents are tested solely for their efficacy to reduce $\mathrm{HbAlc}$, is also largely underappreciated $(53,109,158)$. Finally, traditional methods of assessing glucose are weak in their evaluation of actual glycemic control. Traditional assessment has been dominated by stagnant measures such as fasting blood glucose and $\mathrm{HbAlc}$, measures that do not reflect the glucose fluctuations which occur throughout the day in the free-living diabetic patient. However, postprandial glycemic control and the excursions in glucose after meals not only better depict physiological regulation and the free-living condition when compared to traditional measures, but recent data suggest they also are more predictive of disease risk $(27,28,33,34)$. As the burden of diabetes expands, a greater emphasis on improved assessment strategies and in centralizing exercise in the treatment regime will improve diabetic complications and should reduce morbidity and mortality.

\section{Physiology of normal and impaired PPG}

Although a multitude of factors are involved in metabolic regulation, the liver, pancreas, and skeletal muscle are three primary sites contributing to glycemic control 
following meal ingestion (43). When an individual who is metabolically healthy consumes a meal, the liver shuts down glucose production, the pancreas secretes insulin, insulin signals glucose uptake, and the skeletal muscle takes glucose out of the blood (55). Figure 16, drawn based on classical work from DeFronzo and Ferrannini, illustrates glucose appearance and clearance from the blood following ingestion of glucose or a meal in normal, healthy physiology and how these tissues contribute to postprandial glycemic control $(45,55,57)$.

However, individuals with T2D have perturbed homeostatic control at multiple points in this diagram and thus handle glucose differently than those without T2D (45, $47,55-57)$. Since several of the illustrated organs and tissues (liver, pancreas, and skeletal muscle, among others) are involved in the pathology of T2D, mechanistically, several tissues could be involved at different stages of exercise training in improving glycemic control. 


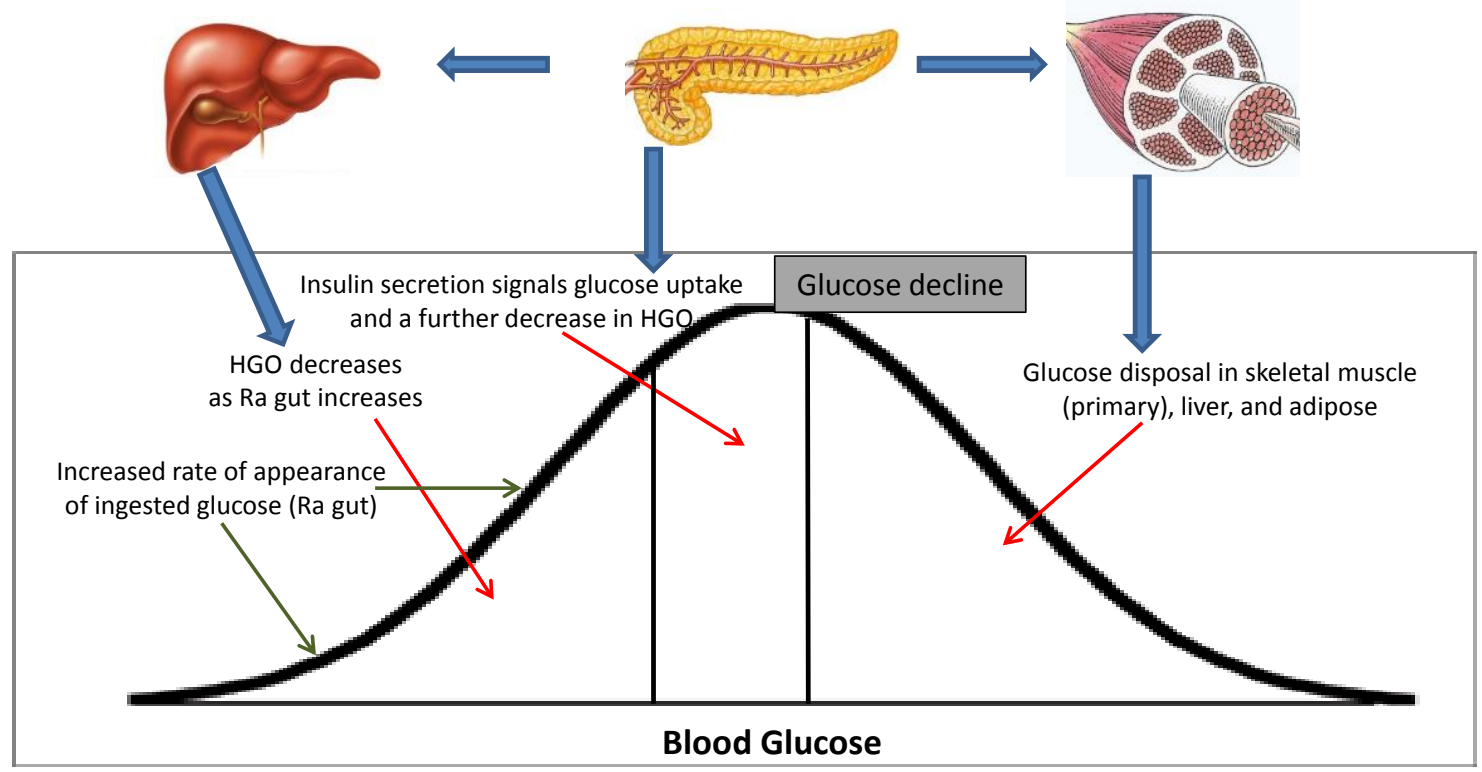

FIGURE 16 - Illustration of the appearance and disposal of a glucose load in healthy subjects. Individuals with T2D have impairments at multiple points in this sequence. 


\section{The development of T2D}

Etiologically, T2D is a complicated disease which involves progressive worsening of glycemic control. As illustrated by Wolever et al. (177), the typical progression from the normal healthy condition to blunt T2D can be generally described by four stages (Figure 17). In the first stage, which is represented by the "Lean" subjects in his study, both plasma glucose and plasma insulin responses to a glucose or meal challenge are normal and return close to fasting levels after two hours. In stage two, which is routinely observed in obese populations, the plasma glucose response to the challenge is still relatively normal, but these subjects experience higher than normal plasma insulin values, or mild hyperinsulinemia. While they are still considered to have normal glucose tolerance, they already exhibit pathology when insulin levels are considered. In the third stage, labeled Impaired Glucose Tolerant, or IGT, there exists overt hyperinsulinemia with impaired glucose tolerance. This is a critical stage, as it illustrates that the pancreatic $\beta$-cells are functioning at a very high capacity but the insulin secreted is not able to maintain normal blood glucose levels primarily due to severe insulin resistance (44). Further, not only is peak blood glucose higher in this phase, but glucose two hours following ingestion of the meal or glucose beverage is still significantly elevated compared to baseline. Finally, stage four shows glucose and insulin responses in overt T2D. Here, pancreatic $\beta$-cell failure is evident, as plasma insulin levels are low with concurrent severe hyperglycemia. While it should be noted that cases of T2D exist that do not follow this progression, or which go through this progression extremely rapidly rather than over a span of several years, they are the exception and may include a substantially larger genetic component than the typical case $(107,126)$. 

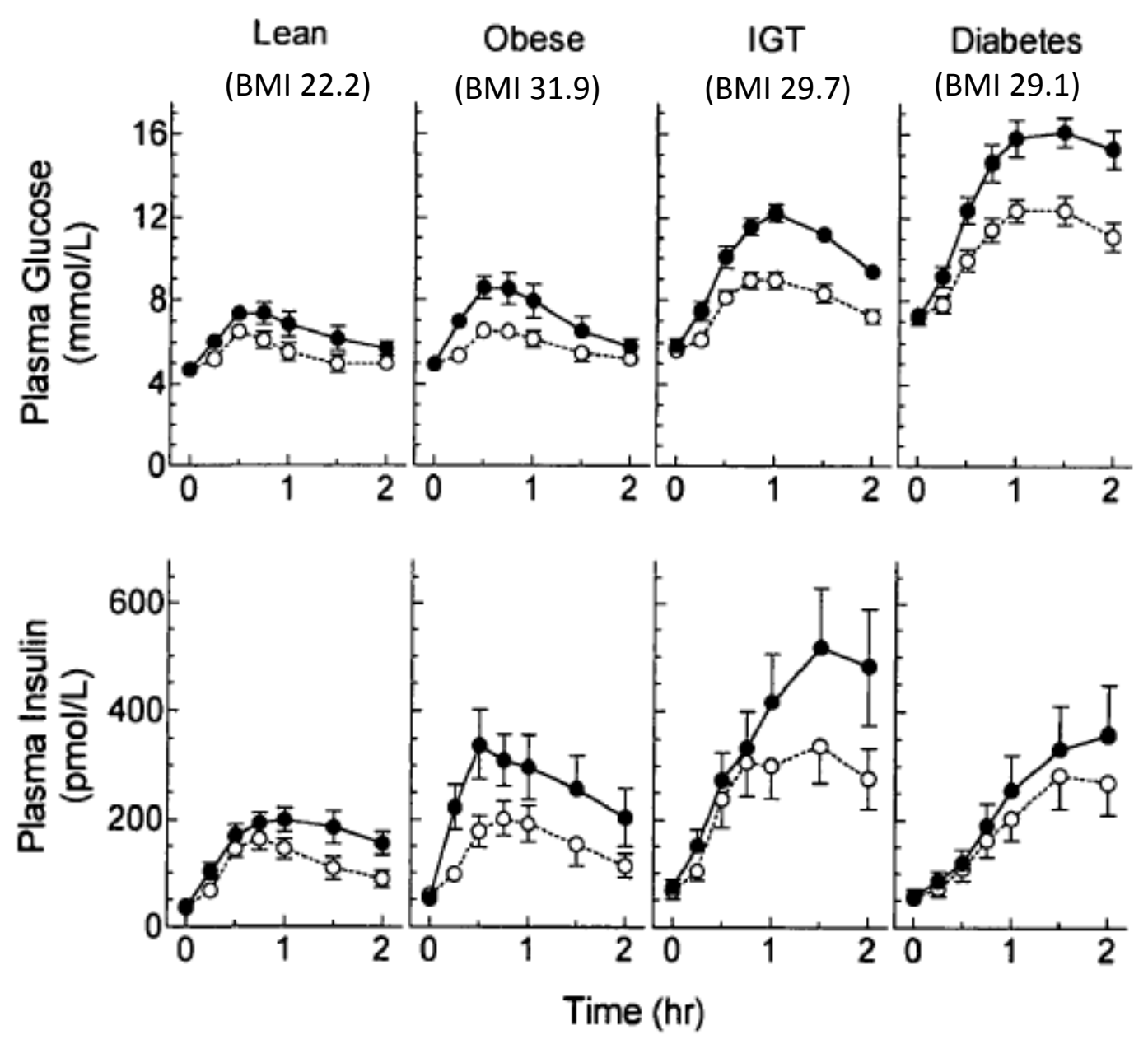

FIGURE 17 - Plasma glucose and insulin levels during an OGTT (closed circles) and during a MMTT (open circles). Points represent means \pm SEM. Figure adapted from Wolever et al., Diabetes Care, 1998(177). 
Genetics, epigenetics, and lifestyle. Both genetic and environmental factors, as well as epigenetics, have been implicated in the development of T2D $(126,127)$. The genetic influences of T2D etiology is a booming area of research, as many links continue to be uncovered. Genome-wide association studies and gene sequencing have allowed huge strides to be made in understanding genetic and environmental interplay in diabetes development, but it is still a complex field with many unknown relationships requiring careful study (126). Further, the array of potential epigenetic influences on T2D development is a relatively nascent area of study but is one that is growing in epidemic proportion due to recognition of the interplay between genetics and environment. Currently, it is understand that epigenetic changes occur which affect both insulin sensitivity and $\beta$-cell function, and these changes are intergenerational (127). However, exactly what these changes are, their lasting effects, and their modifiability have yet to be elucidated.

Although there is generally some underlying genetic predisposition to developing T2D, lifestyle and environment can profoundly impact one's risk for developing T2D (127). Both obesity and sedentary lifestyle are known risk factors for T2D (152). Furthermore, large scale intervention trials demonstrate that reversing inactivity or sedentary lifestyle through exercise, and minimizing obesity through weight loss, can delay the onset of T2D in those patients at highest risk $(90,123,166)$. And, exercise has been shown to improve several physiological abnormalities considered causative, including declines in insulin sensitivity and $\beta$-cell function $(20,82)$. Collectively, this demonstrates that not only do physical activity and body composition causally relate to 
disease progression, but progression is modifiable in many patients if appropriate interventions are undertaken.

Disease progression and utility of exercise as treatment. Controlling glycemia is critical in the management of T2D. Among the intrinsic modalities of glycemic control are insulin secretion and insulin sensitivity (stimulating glucose transport in muscle and inhibiting glucose production in the liver). Insulin is produced by and secreted from the Islets of Langerhans in the pancreatic $\beta$-cells (15). When the $\beta$-cells are functioning appropriately, as in the non-diabetic healthy person, insulin is released in response to a rise in circulating glucose following ingestion of a meal. However, under chronic stress and hyperactive demand, as occur with repeated exposure to hyperglycemia, $\beta$-cells can become dysfunctional. This usually occurs after prolonged periods of time in which hypersecretion of insulin is necessary to overcome insulin resistance. $\beta$-cell dysfunction occurs when the pancreas is no longer able to produce insulin at levels necessary to maintain euglycemia; this defect in insulin secretion is the primary manifestation of T2D (81).

The second insulin-associated contributor to glycemic control is reduced insulin sensitivity, or insulin resistance. Insulin sensitivity is the ability of an organ or tissue to respond to insulin's hormonal signal and is most commonly used to describe insulin's ability to stimulate increased glucose uptake into skeletal muscle and reduce glucose production from the liver (16). Reduced insulin sensitivity generally precedes $\beta$-cell dysfunction and T2D and is observed in both healthy individuals with a family history of diabetes (105) as well as in individuals with pre-diabetes $(4,18)$. Insulin resistance, the pathologic term for reduced insulin sensitivity, can occur at the level of multiple organs 
and tissues, but its effects in skeletal muscle and liver are particularly perilous in T2D (3, 43). Primary maladaptations associated with T2D include the inability of insulin to effectively increase glucose disposal in skeletal muscle and suppress hepatic glucose production during postprandial conditions, factors linked to both chronic physical inactivity and obesity $(86,128,162)$. Fasting hyperglycemia also appears to be linked to hepatic insulin resistance and pancreatic $\beta$-cell dysfunction. However, regular physical activity and exercise attenuate insulin resistance $(24,82)$. Furthermore, as evidence continues to emerge, it is becoming increasingly clear that a sedentary lifestyle with lack of regular exercise is the root cause of insulin resistance $(93,160,161)$. And while it is clear that exercise is beneficial for improving postprandial glycemic control $(98,109$, $121,123)$, the mechanisms through which this occurs are not well understood but are likely related to a combination of factors which improve insulin sensitivity and insulin secretion.

In healthy lean individuals and obese individuals with normal glucose tolerance, insulin secretion and insulin sensitivity have a unique hyperbolic relationship; in individuals who are highly sensitive to insulin, large changes in insulin sensitivity produce only small changes in insulin secretion whereas in those with low insulin sensitivity, even small changes in insulin sensitivity result in large changes in insulin secretion (83). Thus, insulin secretion is extremely important when insulin sensitivity is low. This relationship is also important in understanding the development of impaired glucose tolerance and type 2 diabetes (81). Insulin resistance, or impaired insulin sensitivity, as previously mentioned can initially be offset by increased production of insulin by the pancreatic $\beta$-cells to partially maintain euglycemia. More directly, as 
insulin sensitivity declines, due to prolonged inactivity and obesity, insulin secretion increases to compensate. However, as obesity and physical inactivity continue, insulin sensitivity continues to decrease and insulin secretion reaches a maximal point. In some individuals a hypersecretion of insulin can continue to occur and offset the development of T2D; however, in other individuals $\beta$-cells can no longer maintain hyperinsulinemia leading to gross impairment of glucose homeostasis and the development of $\mathrm{T} 2 \mathrm{D}(80,81)$.

The relationship between insulin sensitivity and insulin production has been described mathematically as the disposition index, which relates insulin secretion to insulin sensitivity (83). Moreover, the disposition index is particularly useful in that it can predict the development of poor glycemic control and future incidence of diabetes (81, $83,105,170)$. Furthermore, the importance of the disposition index is realized as data from two large-scale trials have shown that worsened glycemic control over the duration of diabetes in patients undergoing aggressive treatment from traditional medications can be attributed to continued deterioration of $\beta$-cell function without a change in insulin sensitivity $(2,97,106)$. Thus, while medications may improve hyperglycemia in the short-term, they are not able to reverse or even maintain $\beta$-cell function and ultimately are largely unable to stop diabetes progression. The induction of oxidative stress by hyperglycemia, referred to as glucose toxicity, putatively plays a leading role in impairing $\beta$-cell function leading to diminished insulin production and release during postprandial conditions $(125,135)$. The combination of reduced insulin production and insulin resistance in peripheral tissues leads to greater hyperglycemia in a feed-forward process resulting in even greater oxidative stress and $\beta$-cell dysfunction. 
Since both insulin secretion and insulin sensitivity are paramount to good glycemic control, therapies that target both factors would be most effective to treat T2D. And indeed, unlike medications currently available, this is what exercise does. While drug therapy fails to improve disposition index, exercise training can impact both insulin sensitivity and $\beta$-cell function, working via two arms to improve disposition index and glycemic control (150).

\section{Exercise improves glycemic control}

Early studies. Although it was early in the $20^{\text {th }}$ century that a physician first realized an acute bout of exercise could help lower blood glucose (94), it was not until decades later that exercise's effects on glycemic control truly began to be understood. Seminal work showing that exercise has the ability to improve insulin function and the potential to improve glucose control was performed in the 1970s and early 1980s $(17,71$, $95,99,111)$. One of the earliest studies in humans to suggest a training effect on glucose metabolism came from Per Björtorp and colleagues in 1972 (17). This group observed that when compared to control subjects, trained men had rapid glucose absorption with lower circulating insulin levels, suggesting that physical activity and exercise training are at least partially responsible for regulating plasma insulin and likely affect glucose metabolism. This launched further research into the effects of exercise training on insulin action.

Additional work found that changes in fitness, as measured by maximal oxygen consumption, partially predicted but were not responsible for the effects of exercise training on improving insulin-stimulated glucose disposal and that changes in adiposity 
could be causative $(95,131)$. And indeed, skeletal muscle mass is a major factor in glucose metabolism. To illustrate this, in 1980, Mondon et al. identified in rats that skeletal muscle is the primary site for insulin-stimulated glucose uptake following exercise training(111). This was confirmed in humans when DeFronzo and colleagues identified a year later that skeletal muscle is responsible for more than $85 \%$ of glucose uptake during a hyperinsulinemic-euglycemic clamp performed after exercise(46). And as more evidence emerged, it became increasingly clear that total muscle mass was not the sole cause of improved glucose metabolism, but rather it was moreso enhanced insulin action. In humans, Lohmann and colleagues observed that compared to healthy, untrained subjects, trained distance runners displayed both lower basal circulating insulin concentrations and a lower insulin response to a glucose challenge (99).

Galbo et al. approached this observation mechanistically by exposing primary pancreatic islets isolated from exercise trained rats to physiological glucose concentrations and discerning their insulin secretion (61). They found that compared to control islets from sedentary rats, the islets from exercised rats had lower insulin secretion, indicative of reduced glucose sensitivity. Though this may seem somewhat counterintuitive, lower glucose sensitivity can explain the lower insulin concentrations observed in both animal and human models of exercise training; less insulin is needed for a given glucose concentration and thus less is secreted. Finally, Richter et al. showed, in rats, that exercise can actually reverse insulin resistance and that the effect is most prominent in the working muscles that are depleted of glycogen (132). Repeated studies have since found that acute exercise can reverse muscle insulin resistance (reviewed in Thyfault, 2008) (159). 
Seminal work in humans. Glucose disposal and insulin sensitivity are greatly influenced by skeletal muscle contraction and exercise status. Illustrative of this, a landmark study demonstrating exercise's capability to improve insulin sensitivity and consequently glycemic control in humans came from John Holloszy's lab in 1983. In this study, Heath et al. (70) demonstrated in young, healthy, exercise trained men and women, that removing exercise training bouts for as little as 10 days results in marked increases in circulating insulin with reduced glucose control. However, even a single bout of acute exercise following training cessation restored insulin sensitivity and glycemic control to levels found before exercise cessation occurred. Interestingly, the large rise in insulin with concurrent elevation of glucose following exercise cessation in this study occurred in the absence of changes in maximal oxygen consumption or body composition, indicating that the detraining effects on insulin sensitivity are much faster-acting than traditional factors linked to exercise improved insulin sensitivity. Conversely, they also appear to be transient, as evidenced by the single exercise bout nearly ameliorating the change. Thus, this study showed that not only is continued exercise training important for maintaining glucose control, but even a single exercise stimulus has a pronounced impact on improving metabolic function. Furthermore, additional work in this lab has shown that glucose disposal is not solely dependent on insulin-stimulated uptake and that exercise also triggers increased glucose transport via insulin-independent translocation of the glucose transporter, GLUT4, to cell membranes in skeletal muscle (129). Thus, exercise is effective at improving glucose uptake via both insulin-dependent and insulinindependent pathways, additively improving glycemic control (62). 


\section{Exercise is important for glycemic control in both healthy and diseased states.}

Several cross-sectional studies in addition to prospective clinical trials have shown that physical activity and exercise are associated with improved glucose control and that a sedentary lifestyle is associated with worsened glucose control both in healthy individuals and in those with T2D $(69,93)$. Genevieve Healy and colleagues (69) assessed glucose responses to an OGTT in individuals stratified by activity levels and found that time spent sedentary, lightly active, and moderate to vigorously active is highly related to $2 \mathrm{~h}$ plasma glucose. Further, in data from Bente Pederson's lab, KroghMadsen and colleagues illustrate that when healthy active individuals become physically inactive through reduced daily stepping, their insulin sensitivity and glycemic control are drastically reduced (93).

Physical activity and exercise are paramount in good glycemic control, and exercise training improves whole-body glycemic control. Studies by both Mikus (110) and Reynolds (130) have shown that reducing physical activity in active, healthy subjects for only 3-5 days impairs glycemic control as assessed by a continuous glucose monitoring system (CGMS). While these studies provide insight into the role of physical activity and exercise in maintaining good glycemic control, improving glycemic control in the already diabetic population must also be addressed.

Indeed, the observations from the healthy population can be extrapolated to the insulin resistant and diabetic population. A 2001 meta-analysis (22) examining multiple small scale trials found that exercise training, even without changes in body mass, significantly reduces $\mathrm{HbA} 1 \mathrm{c}$ by a clinically relevant degree: from $8.31 \%$ to $7.65 \%$. More recently, another meta-analysis found that structured aerobic training, resistance training, 
and the combination were all associated with improved HbA1c in patients with T2D (169). Furthermore, as little as seven days of exercise training improves glycemic control assessed by powerful lab-based and free-living measures. Kirwan et al. (89) showed that one week of exercise training improves both peripheral and hepatic insulin sensitivity in patients with T2D. This was evaluated by doing a two-stage hyperinsulinemic euglycemic clamp, a lab-based measure and the gold standard for assessing insulin sensitivity in vivo (48). In this study, glucose disposal with hyperinsulinemia was increased following exercise training during both stages of the clamp, indicating increased glucose uptake in skeletal muscle and improved insulin sensitivity. Additionally, in both the basal condition and with low-dose insulin infusion (40 mU), hepatic glucose production was lower after exercise training compared to before, indicative of improved liver insulin sensitivity. Importantly, these changes in both muscle and liver occurred in the absence of weight loss. Thus, the robust effects of improved insulin action and glucose uptake can be observed after short-term aerobic exercise training and are not due to changes in body weight. Following this, Mikus et al. (108) investigated postprandial glycemic control using CGMS in the free-living condition and found that seven days of exercise training attenuated PPG and glycemic excursions. Hence, the observation by Kirwan that a week of exercise improved glucose uptake as measured in the lab was reflected in the free-living condition as well.

In a separate study from Kirwan's lab, Solomon et al. found that in obese prediabetic subjects, exercise combined with a low glycemic index diet improved insulin sensitivity and reduced the postprandial insulin response, suggestive of enhanced glycemic control in this population (149). Thus, exercise is a valuable therapeutic option 
for patients with T2D. However, the mechanisms and extent of these improvements, as well as the impact on different tissues, including the $\beta$-cell, skeletal muscle, liver, and adipose, remain unknown. In addition, when along the time course of exercise training positive changes occur in various tissues and how these relate to glucose control at the whole-body level is not known.

\section{Potential mechanisms whereby exercise improves glycemic control. Initial}

mechanisms of improved glycemic control with exercise are through improved skeletal muscle glucose uptake, especially insulin-stimulated glucose uptake (176). This is principally attributed to increased muscle insulin sensitivity and appears to be restricted to the muscles being used for the exercise bout and linked to glycogen depletion in these muscles $(132,133,176)$. In subjects with impaired glucose tolerance and T2D, GLUT4 protein content is increased in skeletal muscle after short-term exercise training $(1,72$, 76). Insulin signaling is likely also improved, although the evidence for the latter is equivocal in subjects with T2D $(36,37,60,73,171,181)$. Together, this results in improved insulin-stimulated glucose uptake via improved insulin signaling and enhanced translocation of GLUT4 to cell membranes.

Additionally, during exercise, blood glucose is used at a higher rate than at rest, resulting in immediate improvements in hyperglycemia simply by utilizing glucose in the blood for energy. Following acute exercise, particularly that of moderate to higher intensities, hyperglycemia continues to be blunted for $24-48$ hours $(49,50,176)$. This is due to a contraction-induced increase in GLUT4 translocation as well as to the muscle being more sensitive to an insulin stimulus, resulting in improved ability of insulin to signal translocation of GLUT4 to the cellular membrane $(66,138)$. However, the 
reduction in insulin resistance following exercise is short-lived (1-2 days), and current evidence would suggest that exercise should be continued at least every other day to maintain improvements. In addition to this somewhat transient effect, other mechanisms and tissues are also believed to contribute to the improved glycemic control seen with exercise, and this is where research is incomplete.

As the site of insulin production and secretion, the $\beta$-cell is an obvious target in T2D treatment. As shown by Kirwan and in further work by Solomon, aerobic exercise improves $\beta$-cell function and the disposition index $(85,102,150,151)$. This is novel, as most traditional therapies are not able to improve disposition index. Furthermore, the improved $\beta$-cell function seen with exercise training translates to improved glycemic control. Still, the mechanisms by which exercise improves $\beta$-cell function are not clear and should be an area of further investigation. Chronic oxidative stress may be a central mechanism for $\beta$-cell failure in patients with $\mathrm{T} 2 \mathrm{D}(35,135,136)$. Thus, by reducing oxidative stress through exercise training, pancreatic $\beta$-cells would putatively have improved insulin secretion contributing to improvements in glycemic control.

While there is some in vitro evidence and evidence in vivo in mice that dietary antioxidants could reduce oxidative stress (96), studies in humans have not been promising (124). In contrast, exercise is a powerful stimulus which over time results in decreased measures of oxidative stress and improved cellular function (119). It is thought that different isoforms of NADPH oxidase may be a prime regulator of $\beta$-cell function and thus insulin production (118). While acute exercise transiently increases oxidative stress, exercise training leads to upregulation of pathways that protect against oxidative stress $(42,58)$. However, how much exercise is needed to reduce oxidative stress to a 
level resulting in improved $\beta$-cell function and insulin secretion, how this happens, and how long it would take for these effects to emerge remain unknown. It also may be that exercise lowers oxidative stress in T2D patients secondary to an improvement in glycemic control that is primarily driven by improvements in the insulin sensitivity of muscle and liver, a hypothesis that deserves testing.

The liver is another major contributor to glycemic control and is thus another strong target for how exercise mechanistically improves glycemic control. As a central hub for metabolism, the liver integrates substrate and endocrine signals to maintain metabolic homeostasis, particularly in the regulation of blood glucose. In humans, the liver is responsible for the majority of endogenous glucose production, with the kidneys contributing a small amount (59). In fact, the liver contributes such a large degree that the term hepatic glucose production (HGP) is used nearly synonymously with endogenous glucose production. HGP consists of two components: gluconeogenesis, or production of "new" glucose from non-carbohydrate substrates such as fatty acids, and glycogenolysis, or glucose coming from hydrolysis of glycogen. In patients with T2D, the ability of the liver to adequately control these processes is mitigated, resulting in an unwarranted increase in hepatic glucose output (175). However, exercise decreases HGP in healthy people (38) as well as in those with T2D $(63,175)$, both during fasting and during insulin stimulated conditions, ultimately improving the liver's control over regulation of blood glucose. 


\section{Strengths and weaknesses of traditional measures of glycemic control}

Chronic hyperglycemia has long been thought to contribute to increased risk of morbidity and mortality $(40,172)$. However, new emerging data suggest that traditional measures of glycemia are weak in their assessment of true glycemic control, and as stagnant or chronic measures, they are not the best available indicators of morbidity related to dysfunction in glycemic control.

$\boldsymbol{H b A l c}$. Glucose control in T2D is most often assessed by a chronic measure, $\mathrm{HbAlc}$. HbAlc values have been associated in numerous studies with higher rates of cardiovascular disease and death $(40,145,154)$, and current recommendations for controlling glycemia in patients with T2D are to achieve HbA1c levels less than 7\% (8, 147), as levels less than 7\% are associated with fewer microvascular and macrovascular complications in T2D $(63,64,153,154)$.

While a relationship between $\mathrm{HbA} 1 \mathrm{c}$ and blood glucose was originally identified in the middle of the $20^{\text {th }}$ century (65), knowledge of the extent of this relationship has evolved significantly since then, due in part to two large scale studies. The Diabetes Control and Complications Trial (DCCT) (1) and the U.K. Prospective Diabetes Study (UKPDS) (167), both published in the 1990s, truly established the relationship between HbA1c and diabetic complications in both type 1 diabetes and T2D. Yet, chronic measures of glycemic control do not illuminate the importance of fluctuations in glucose control throughout the day. In 2002, data from the DCCT were used to examine the relationship between plasma glucose and HbA1c (140) in 1439 patients with type 1 diabetes from 29 centers across the United States and Canada. This study found that while $\mathrm{HbA} 1 \mathrm{c}$ correlates and is reflective of mean plasma glucose, the relationship is 
complex. For example, pre-lunch and earlier plasma glucose values showed lower correlations than post-lunch and later values. This illustrates that while HbAlc may be a good indicator of chronic glucose averages, it is not sensitive to fluctuations throughout the day and for varying responses to meals.

As newer evidence has shown, though HbA1c is undoubtedly still an important correlate and large scale screening tool, it may no longer be the best measure for determining risk for T2D or risk for cardiovascular disease in those with T2D $(27,28,32$ 34, 112-114, 164). Furthermore, extreme shifts in glycemia also are associated with increased rates of death and disease, often more so than measures of chronic glycemia $(33,88,114)$. Thus, although HbA1c still has clout as a quick identifier of chronic glycemia, other measures should be considered when screening, diagnosing, and treating those at risk for T2D.

Fasting blood glucose. In the clinical setting, fasting blood glucose is also frequently used in assessing glycemia. However, this is not a true measure of glycemic control for several reasons. First, it ignores insulin concentrations, the primary modulator of glucose uptake and hepatic glucose output. Additionally, fasting glucose does not show how the body actually handles glucose; rather, it simply shows basal levels in that individual, providing no real indication of what will happen when the subject consumes a meal. Finally, while T2D can be defined by fasting blood glucose cutoff points, using either $\mathrm{HbA} 1 \mathrm{c}$ alone or fasting blood glucose alone is a poor predictor of impaired glucose tolerance, a condition which can precede and predict susceptibility for T2D $(26,75)$. Another assessment which could be utilized in the clinical setting is measuring fasting insulin in the blood. This is routinely done in research. When both glucose and insulin 
measures are combined, the researcher has both a snapshot of glycemia and an estimate of insulin sensitivity (e.g., using values for HOMA and QUICKI), an important component of glycemic control. Still, the question of how the patient's body actually responds to a stimulus such as feeding cannot be answered using these simplistic methods.

OGTT. Another measure of glycemic control often used in research but less frequently in diagnostic settings is the OGTT. In this test a bolus of glucose, most commonly 75 grams, is ingested in liquid form and blood is sampled two hours later for measurement of glucose concentration. In the clinic, this is generally the extent of the test, but research often utilizes frequent blood sampling and measurement of both glucose and insulin concentrations. Compared to stagnant measures, the OGTT adds the benefit of a glucose challenge. However again, unless insulin is measured in addition to glucose, this test provides only a partial view of how the body is controlling glucose. And, even when both glucose and insulin are measured, this test still has limitations due to using a pure glucose load, which misrepresents normal human feeding encountered on a daily basis. Indeed, meals are almost never pure glucose or rarely even pure sucrose; they generally consist of a mixture of carbohydrates (different mono- and polysaccharides) combined with different fats and protein sources, in addition to both soluble and insoluble dietary fiber that exists naturally in many sources of carbohydrate.

The glycemic index of foods and the glycemic load of a meal depict differences in absorption, as absorption is slowed when fat and protein are added to simple sugars (12, $21,79,100,101)$. Thus, the glycemic load of given meal in the free-living condition will differ greatly from that of the OGTT beverage. Consequently, while the OGTT has the 
benefit of providing a somewhat prandial "challenge," the challenge is not to a real meal and does not represent the free-living condition and how the body actually responds to feeding.

Ultimately, glycemic control is important because of its association with morbidity and mortality, particularly with respect to cardiovascular diseases. Thus, the measures used for examining glycemic control in both the research and clinical settings should both be accurate and relate well to predicting disease. However, traditional measures, while still useful for population-based widespread screening due to their ease and low cost, may now be outdated in effectively predicting disease in at risk patients. Rather, post-challenge glucose levels and glucose spikes after a challenge are increasingly proving to be more predictive of measures of cardiovascular disease such as intima media thickness (155). Yet, the commonly used challenge test, the OGTT, does not mimic human feeding behaviors and thus lacks the physiological relevance of mixed meals containing carbohydrate, protein, and fats that are normally consumed in the freeliving condition. Therefore, methods which better assess free-living glycemic control should be examined.

\section{Utility of assessing free-living glycemic control}

Identifying responses in a laboratory provides the benefit of a controlled environment; however, it lacks the real-life application of observing humans in their natural state. Thus, methods that assess glycemic control in the free-living environment or simulate the free-living condition would provide insight into glycemic control patterns. 
The MMTT and the CGMS are two methods that show promise in assessing free-living glycemic control.

MMTT. The OGTT is one example of a laboratory-based measure that is not characteristic of a human's free-living condition. However, while it does not represent what occurs when a person consumes a standard meal outside of the lab, it is still the most used challenge test in scientific and clinical diabetes literature and the only challenge-based test used to diagnose and classify diabetes or risk for diabetes $(7,9)$. Its strength lies in that it provides a carbohydrate challenge to the body, but a more physiological MMTT, in which carbohydrates, protein, and fat are ingested in the form of a meal (in either solid or liquid composition), would be preferential in determining what responses occur when subjects eat normal meals in the free-living condition. This would mimic the typical meal situation and add further validity to applying lab-based testing to the free-living situation. Yet, while the MMTT has theoretical strength, it is difficult to standardize and has not been validated against other measures, including the OGTT. While a few studies have attempted to evaluate glycemic control using both an OGTT and a MMTT $(25,134,165)$, each has its limitations.

One of the first studies to compare the OGTT and the MMTT was actually designed to evaluate a specific phenomenon, reactive hypoglycemia (25). It was observed that one of the negative consequences of using the OGTT to evaluate glycemic control was that subjects would become hypoglycemic. Thus, the authors of this study sought to determine if a more natural meal test would still have this effect. Twenty-six individuals identified to have symptoms suggestive of reactive hypoglycemia were given an OGTT, and nearly $40 \%$ developed hypoglycemia during the test. The subjects who developed 
hypoglycemia during the OGTT were then administered a MMTT which included 78 grams of carbohydrate, 19 grams of protein, and 15 grams of fat. Interestingly, none of the subjects administered the mixed-meal test developed reactive hypoglycemia. Thus, the authors concluded that hypoglycemia following ingestion of concentrated sugar is uncommon following a mixed meal, and symptoms of hypoglycemia in the free-living state can likely be avoided by consuming mixed meals rather than concentrated carbohydrates. The outcomes of this relatively cursory study design have great implications for human health and the design of subsequent metabolic research studies. If the OGTT is not representative of what occurs in the free-living environment and does not reflect responses in glycemic control observed when a mixed meal is consumed, perhaps our standards for evaluating glycemic control should shift towards the more physiological design.

Other studies also have investigated the utility of using a MMTT. Traub et al. (165) compared blood glucose responses to three different types of tests: a standard OGTT (75grams of glucose; $300 \mathrm{kcal}$ ), a MMTT consisting of a $600 \mathrm{kcal}$ shake [50\% of energy from carbohydrate (75 grams of glucose), 30\% energy from fat, and 20\% energy from protein], and a "muffin test" consisting of a commercially prepared breakfast muffin (average $410 \mathrm{kcal} ; 56$ grams of carbohydrate, 18 grams of fat, 6 grams of protein) plus either coffee or tea. However, this study had several limitations. Notably, the energy contents of the three meals varied greatly, with the mixed-meal test containing double the caloric content of the OGTT. Additionally, the only outcome measure was 2 hour blood glucose; neither insulin nor c-peptide was assessed, offering no real insight into insulin secretion or insulin sensitivity responses and thus true glycemic control. In another study 
from 2009, Rijkelijkhuizen and colleagues found the MMTT to have greater sensitivity in detecting $\beta$-cell response compared to OGTT (134) when equal amounts of carbohydrate were consumed. However, the mixed-meal test used in this study contained grossly more total energy (approximately $833 \mathrm{kcal}$ in the MMTT compared to $300 \mathrm{kcal}$ in the OGTT) and was also very high in fat ( $52 \%$ of the energy content of the meal). Therefore, this test did not show what the response would be if the MMTT were to be more closely matched in total energy to the OGTT.

Finally, perhaps the most relevant study comparing the OGTT and the MMTT was done by Wolever and colleagues (177). This group used the standard OGTT (75 grams of glucose; $300 \mathrm{kcal}$ ) and a MMTT consisting of $345 \mathrm{kcal}$ (including 50 grams of carbohydrate) and examined the reproducibility of each test. Although they did not match the tests for glucose, the total energy content of the tests was fairly close, with the mixedmeal test having only $45 \mathrm{kcal}$ more than the OGTT. The independent natures of these two tests makes comparing them difficult, as it is not possible to match both carbohydrate content and total energy since the mixed meal must also contain fats and protein. However, Wolever's group took the approach of increasing total energy slightly while decreasing carbohydrates in the MMTT as compared to the OGTT. They found that the reproducibility of the MMTT was no different than that of the OGTT for healthy people and those with T2D, and its precision was actually better than the OGTT in the impaired glucose tolerance range. They also found that the mixed-meal test produced fewer negative side effects compared to the OGTT (including less stomach discomfort, hunger, and nausea). 
While this study has many strengths, it should be noted that the authors did not examine $\beta$-cell function, measures of insulin sensitivity, or any measures of or relating to glycemic control other than blood glucose and insulin concentrations. Furthermore, the authors did not evaluate whether or not each test would differ in its ability to detect changes in glycemic control with an intervention such as exercise training. Finally, the meal used in this study was one example of a mixed meal and was provided in the form of a dry wafer; no standards currently exist as to what should constitute a mixed meal challenge.

Each of the current studies leaves remaining questions; and, furthermore, to the researcher's knowledge, no study has examined how the MMTT and the OGTT compare following exercise training, which as outlined earlier, is a critical therapy for improving postprandial glycemic control. Thus, while the MMTT is a promising measure, additional research is necessary to validate it against the OGTT and to compare measures of glycemic control between the two tests in response to an intervention.

CGMS. Another measure of glycemic control gaining popularity is CGMS. The CGMS utilizes a small catheter inserted subcutaneously in the abdomen and measures interstitial glucose concentrations on a continuous basis throughout the day. A prominent strength of CGMS is that it can be easily used outside of the lab, in a subject's free-living environment. Thus, CGMS allows 24 hour monitoring in normal life and will show alterations in glycemia throughout the day, helping us understand a person's normal glucose responses to meals and how they are altered with diet and exercise interventions. When using CGMS in combination with standardized meals and monitored exercise 
sessions in which timing of meal ingestion and exercise is marked, direct outcomes can be assessed and comparisons made between pre- and post-intervention.

Because of its ability to constantly monitor glucose during a patient's normal daily routine, CGMS more accurately reflects what is actually happening to patients' glucose levels than do laboratory based tests. In addition to average glucose levels, CGMS data provide glucose peaks and nadirs, episodes of and time spent in hypoglycemia and hyperglycemia, standard deviation of glycemia, and mean amplitudes of glycemic excursions, all of which give an understanding of how glucose fluctuations differ throughout the day and in response to exercise. However, a limitation is that the device only measures glucose. Thus, while it provides an exceptional view of this key component of glycemic control, it does not provide any estimation of insulin sensitivity or $\beta$-cell function, which are key elements in controlling glycemia.

\section{Postprandial glucose and postprandial glucose excursions}

Glycemic variability is closely related to disease. Indeed, both hyper- and hypoglycemic episodes are associated with increased morbidity and mortality, particularly from cardiovascular diseases, and hyperglycemic spikes, including those which remain elevated hours after a meal, are particularly detrimental $(28,33)$. Postmeal, or post-challenge, hyperglycemia in particular is a good predictor of cardiovascular disease mortality and has been shown to be a better predictor of cardiovascular morbidity and mortality than either HbA1c or fasting glucose (27-29, 143). This measure, which assesses how well the body clears glucose from the bloodstream, is generally taken two hours following a challenge such as administration of an oral bolus of glucose (13) and is 
predictive of both all-cause mortality and cardiovascular disease mortality, independent of other risk factors for cardiovascular disease (143).

Although reducing $\mathrm{HbA} 1 \mathrm{c}$ in diabetic patients has been and continues to be a standard goal for practitioners, recent evidence indicates that lowering average blood glucose alone does not always result in reduced morbidity and mortality $(51,64,147$, 156). Indeed, several large scale trials examining aggressive pharmacotherapy to reduce HbA1c in patients with T2D to levels closer to those of healthy persons have yielded intriguing results and raised vital questions. The ACCORD (Action to Control Cardiovascular Risk in Diabetes) Trial compared intensive glycemic control, aimed at decreasing $\mathrm{HbA} 1 \mathrm{c}$ below $6 \%$, to standard glycemic control, aimed at achieving an $\mathrm{HbA} 1 \mathrm{c}$ of 7-7.9\% (64). In doing this, the study sought to determine whether aggressively lowering circulating glucose in patients with $\mathrm{T} 2 \mathrm{D}$ would result in fewer cardiovascular events, thus reducing morbidity and potentially mortality in these patients. Surprisingly, the opposite occurred, and the study was consequently terminated early. Patients in the intensive glycemic control group experienced episodes of fluctuating glycemia and hypoglycemic episodes, providing further evidence that fasting glucose and $\mathrm{HbA1c}$ alone are not good measures of overall control of glucose throughout the day. In the ADVANCE (Action in Diabetes and Vascular Disease: Preterax and Diamicron Modified Release Controlled Evaluation) study, intensive therapy also significantly reduced HbA1c. And, though the number of combined macrovascular and microvascular events was fewer in the intensive therapy group compared to control, there was no difference in major macrovascular events and no difference in cardiovascular or all-cause mortality (156) Further, there were more hypoglycemic episodes in the intensive therapy group 
compared to control, similar to what was observed in the ACCORD study. These trials further illustrate that HbA1c may not be the best assessment of glycemic control and that other clinical targets may be necessary to improve cardiovascular end points.

\section{Medication and exercise interventions in treating T2D}

Traditional pharmaceutical therapies. While both pharmaceutical and lifestyle interventions are recommended to improve glycemic control in patients with $\mathrm{T} 2 \mathrm{D}$, the clinical focus is currently on medication rather than lifestyle therapies such as exercise. As such, there is an assortment of medications available to manage hyperglycemia. These therapies range from insulin sensitizers (including thiazolidinediones), insulin secretion enhancers (sulfonylureas), glucagon-like peptide 1 (GLP-1) agonists, dipeptidyl peptidase IV (DPP-IV) inhibitors, $\alpha$-glucosidase inhibitors, sodium-glucose cotransporter 2 (SGLT2) inhibitors, biguanides, and metformin $(19,117)$. Insulin is also routinely prescribed in advanced or poorly-managed disease states, or temporarily just after diagnosis (168). The biguanide Metformin, the most commonly prescribed medication for $\mathrm{T} 2 \mathrm{D}$ at present, is thought to act primarily through the liver to decrease endogenous glucose production and thus decrease overall blood glucose levels. As a first-line treatment, it is often prescribed with at least one other medication.

A newer pharmaceutical approach for T2D targets incretin hormones, including GLP-1 and gastric inhibitory polypeptide (GIP) (11). GIP and GLP-1 are the major insulin-stimulating hormones thought to impact PPG. GLP-1 is released primarily from L-cells in the distal ileum and colon in response to feeding, while GIP is generally secreted from more proximal areas of the digestive tract, primarily K-cells of the small 
intestine (duodenum and jejunum). Together, these hormones are secreted in response to a meal and are understood to signal insulin release, suppress glucagon secretion, and prolong gastric emptying $(11,163)$. Although treatments for GIP have not been promising, GLP-1 continues to remain a prime target. Exendin-4 is a popular GLP-1 agonist used in treatment of T2D, and DPP-IV inhibitors also are routinely used to inhibit the breakdown of GLP-1 $(67,91)$. Since these hormones appear to be integral in regulating insulin secretion, whether or not they are affected by exercise is a valid question. And, the effect of exercise on both GIP and GLP-1 has been examined repeatedly. However, results are equivocal and many studies show no change $(54,173)$. Finally, the latest medications released for treating T2D are SGLT2 inhibitors (30). These drugs, known as canagliflozin and dapagliflozin, act by increasing urinary excretion of glucose and in so doing also result in mild weight loss. Though several clinical trials have assessed short-term safety, there are developing concerns regarding the long-term safety of SGLT2 inhibitors, and this remains to be seen.

However, the long-term efficacy of traditional drug therapy is not promising (106, 167, 168). Additionally, the safety of long-term treatment with several common diabetic medications is also debatable. As one example, both insulin therapy and treatment with sulfonylureas have been linked with increased cancer incidence in people with T2D (23). Additionally, a 2007 meta-analysis found that use of rosiglitazone for greater than 12 months was associated with increased risk of myocardial infarction and heart failure in patients with T2D (146). Even though risks have been identified in these drugs as well as in others, they continue to be used, as no one pharmacological agent is effective for 
treating glycemia in this population. Coincident with this, many patients require a multidrug approach to manage their disease.

With all of these options, an intricate knowledge of both the patient and their disease management must be considered when prescribing medications, and as monotherapy alone typically is not effective, the patient with T2D is often prescribed a cocktail regimen to manage his or her disease (168). In addition, a cacophony of drugs is used to manage common diabetic comorbidities, including dyslipidemia and hypertension. These may be prescribed in combination with lifestyle intervention therapy, but lifestyle intervention is rarely the focus of appointments.

Exercise is a valid option for treating T2D. Although medications are critical in achieving disease management in some patients with T2D, all available pharmaceutical therapies for T2D have side effects, and there is currently no truly effective drug therapy for stopping disease progression. Thus, a continued search for an effective therapy that both manages glycemia and blunts the progression of T2D is needed. And, exercise may be that therapy. Physical activity and exercise of varying intensities have shown to be effective in improving glucose control in many research trials $(69,70,74,82)$. However, even when lifestyle intervention is used in the treatment regime, weight loss is still the primary lifestyle intervention prescribed. However, weight loss has shown to be either difficult to achieve, particularly in people with T2D (174), or is short-lived (a majority of patients who achieve measurable weight loss re-gain the lost weight) (92). But, exercise even in the absence of weight loss, particularly when employed early after diagnosis with T2D, is effective in lowering glycemia, improving glucose handling, and reducing or in some cases even obliterating the need for drug treatments for people with T2D (158). In 
addition, contrary to what is seen with pharmaceutical interventions, any side effects observed with exercise are typically positive, including increased fitness, decreased fatigue, increased sense of well-being, and weight loss or maintenance, among others (157).

Indeed, habitual exercise is an operative treatment for T2D. However, with all of its known benefits, it is still under-utilized in treatment of T2D. Reasons for this could be numerous but should be addressed in order to improve treatment outcomes. First, although exercise's profound impact on glycemic control and PPG is well-known in the metabolic research community, the knowledge has not translated to effectively influence policy. Thus, the medical community is not encouraged to prescribe exercise as a firstline treatment. Second, the design of the current health care system is not conducive to lifestyle intervention. Even when exercise is recommended by physicians, it is generally a very brief part of the conversation.

Another barrier to effective exercise prescription for T2D is a lack of referral to appropriate specialists. Currently, physicians and sometimes diabetes educators are the only personnel giving lifestyle recommendations, and they are not trained to effectively prescribe or administer exercise to their patients $(77,122)$. Further, the terms physical activity and exercise are used interchangeably in recommendations, but there are distinct differences between these, and how recommendations are presented can affect how they are adopted by patients as well as how effective they are (122). Thus, a model is needed where physicians would refer diabetic patients for exercise prescription and training, much like what is done with cardiac patients undergoing cardiac rehabilitation. This has 
been suggested before, and indeed, not only would it benefit the patient's health, but it would be a cost-effective treatment $(122,169)$.

A third obstacle to exercise being routinely prescribed is the incompleteness of knowledge of the mechanisms by which it acts to improve health in T2D. Thus, longterm studies are still needed to determine if exercise blunts disease progression more effectively than drug treatment, and how exercise mechanistically works through multiple organs and tissues to synergistically improve glycemic control.

\section{Summary}

Exercise is integral in treating PPG and overall glycemic control in patients with T2D. Since T2D is a major health care concern for which pharmaceutical treatment provides only moderate management of outcomes, lifestyle therapies such as exercise are needed and may provide critical therapeutic control. Furthermore, PPG and PPGE better predict cardiovascular outcomes than do stagnant measures of glycemia. Because of this, responses to meals in the free-living condition should be central in our assessment and monitoring of changes in glycemic control. Since exercise reduces PPG and improves glycemic control, a better understanding of how this occurs could enhance the use of exercise in treating T2D. To improve our understanding of exercise's effects in freeliving humans, techniques which assess PPG should be employed using methodology applicable to subjects' natural environments. 


\section{REFERENCES}

1. The effect of intensive treatment of diabetes on the development and progression of long-term complications in insulin-dependent diabetes mellitus. The Diabetes Control and Complications Trial Research Group. The New England Journal of Medicine. 1993;329(14):977-86.

2. Intensive blood-glucose control with sulphonylureas or insulin compared with conventional treatment and risk of complications in patients with type 2 diabetes (UKPDS 33). UK Prospective Diabetes Study (UKPDS) Group. Lancet. 1998;352(9131):837-53.

3. Abdul-Ghani MA, Matsuda M, Balas B, DeFronzo RA. Muscle and liver insulin resistance indexes derived from the oral glucose tolerance test. Diabetes Care. 2007;30(1):89-94.

4. Abdul-Ghani MA, Tripathy D, DeFronzo RA. Contributions of beta-cell dysfunction and insulin resistance to the pathogenesis of impaired glucose tolerance and impaired fasting glucose. Diabetes Care. 2006;29(5):1130-9.

5. American Diabetes Association. Diagnosis and classification of diabetes mellitus. Diabetes Care. 2011;34 Suppl 1:S62-9.

6. American Diabetes Association. Economic costs of diabetes in the U.S. in 2012. Diabetes care. 2013;36(4):1033-46.

7. American Diabetes Association. Diagnosis and classification of diabetes mellitus. Diabetes Care. 2014;37 Suppl 1:S81-90. 
8. American Diabetes Association. Executive summary: Standards of medical care in diabetes--2014. Diabetes Care. 2014;37 Suppl 1:S5-13.

9. American Diabetes Association. Standards of medical care in diabetes--2014. Diabetes Care. 2014;37 Suppl 1:S14-80.

10. Azuma K, Kawamori R, Toyofuku Y et al. Repetitive fluctuations in blood glucose enhance monocyte adhesion to the endothelium of rat thoracic aorta. Arterioscler Thromb Vasc Biol. 2006;26(10):2275-80.

11. Baggio LL, Drucker DJ. Biology of incretins: GLP-1 and GIP. Gastroenterology. 2007;132(6):2131-57.

12. Bantle JP, Laine DC, Castle GW, Thomas JW, Hoogwerf BJ, Goetz FC. Postprandial glucose and insulin responses to meals containing different carbohydrates in normal and diabetic subjects. The New England Journal of Medicine. 1983;309(1):7-12.

13. Bartoli E, Fra GP, Carnevale Schianca GP. The oral glucose tolerance test (OGTT) revisited. European Journal of Internal Medicine. 2011;22(1):8-12.

14. Basu A, Dalla Man C, Basu R, Toffolo G, Cobelli C, Rizza RA. Effects of type 2 diabetes on insulin secretion, insulin action, glucose effectiveness, and postprandial glucose metabolism. Diabetes Care. 2009;32(5):866-72.

15. Baudry A, Leroux L, Jackerott M, Joshi RL. Genetic manipulation of insulin signaling, action and secretion in mice. Insights into glucose homeostasis and pathogenesis of type 2 diabetes. EMBO Rep. 2002;3(4):323-8. 
16. Bergman RN, Bucolo RJ. Interaction of insulin and glucose in the control of hepatic glucose balance. The American Journal of Physiology. 1974;227(6):131422.

17. Bjorntorp P, Fahlen M, Grimby G et al. Carbohydrate and lipid metabolism in middle-aged, physically well-trained men. Metabolism: Clinical and Experimental. 1972;21(11):1037-44.

18. Bock G, Dalla Man C, Campioni M et al. Pathogenesis of pre-diabetes: mechanisms of fasting and postprandial hyperglycemia in people with impaired fasting glucose and/or impaired glucose tolerance. Diabetes. 2006;55(12):353649.

19. Bolen S, Feldman L, Vassy J et al. Systematic review: comparative effectiveness and safety of oral medications for type 2 diabetes mellitus. Annals of Internal Medicine. 2007;147(6):386-99.

20. Borghouts LB, Keizer HA. Exercise and insulin sensitivity: a review. International Journal of Sports Medicine. 2000;21(1):1-12.

21. Borgstrom B, Dahlqvist A, Lundh G, Sjovall J. Studies of intestinal digestion and absorption in the human. The Journal of Clinical Investigation. 1957;36(10):1521-36.

22. Boule NG, Haddad E, Kenny GP, Wells GA, Sigal RJ. Effects of exercise on glycemic control and body mass in type 2 diabetes mellitus: a meta-analysis of controlled clinical trials. JAMA. 2001;286(10):1218-27. 
23. Bowker SL, Majumdar SR, Veugelers P, Johnson JA. Increased cancer-related mortality for patients with type 2 diabetes who use sulfonylureas or insulin: Response to Farooki and Schneider. Diabetes Care. 2006;29(8):1990-1.

24. Bunprajun T, Henriksen TI, Scheele C, Pedersen BK, Green CJ. Lifelong Physical Activity Prevents Aging-Associated Insulin Resistance in Human Skeletal Muscle Myotubes via Increased Glucose Transporter Expression. PloS One. 2013;8(6):e66628.

25. Buss RW, Kansal PC, Roddam RF, Pino J, Boshell BR. Mixed meal tolerance test and reactive hypoglycemia. Hormone and metabolic research $=$ Hormon- und Stoffwechselforschung $=$ Hormones et metabolisme. $1982 ; 14(6): 281-3$.

26. Carson AP, Reynolds K, Fonseca VA, Muntner P. Comparison of A1C and fasting glucose criteria to diagnose diabetes among U.S. adults. Diabetes Care. 2010;33(1):95-7.

27. Cavalot F, Pagliarino A, Valle M et al. Postprandial blood glucose predicts cardiovascular events and all-cause mortality in type 2 diabetes in a 14-year follow-up: lessons from the San Luigi Gonzaga Diabetes Study. Diabetes Care. 2011;34(10):2237-43.

28. Cavalot F, Petrelli A, Traversa M et al. Postprandial blood glucose is a stronger predictor of cardiovascular events than fasting blood glucose in type 2 diabetes mellitus, particularly in women: lessons from the San Luigi Gonzaga Diabetes Study. The Journal of Clinical Endocrinology and Metabolism. 2006;91(3):813-9. 
29. Cederberg H, Saukkonen T, Laakso M et al. Postchallenge glucose, A1C, and fasting glucose as predictors of type 2 diabetes and cardiovascular disease: a 10year prospective cohort study. Diabetes Care. 2010;33(9):2077-83.

30. Cefalu WT, Riddle MC. SGLT2 Inhibitors: The Latest "New Kids on the Block"! Diabetes Care. 2015;38(3):352-4.

31. Centers for Disease Control and Prevention. National Diabetes Statistics Report. 2014.

32. Ceriello A. Postprandial hyperglycemia and diabetes complications: is it time to treat? Diabetes. 2005;54(1):1-7.

33. Ceriello A, Esposito K, Piconi L et al. Oscillating glucose is more deleterious to endothelial function and oxidative stress than mean glucose in normal and type 2 diabetic patients. Diabetes. 2008;57(5):1349-54.

34. Ceriello A, Hanefeld M, Leiter L et al. Postprandial glucose regulation and diabetic complications. Archives of Internal Medicine. 2004;164(19):2090-5.

35. Ceriello A, Motz E. Is oxidative stress the pathogenic mechanism underlying insulin resistance, diabetes, and cardiovascular disease? The common soil hypothesis revisited. Arterioscler Thromb Vasc Biol. 2004;24(5):816-23.

36. Christ-Roberts CY, Pratipanawatr T, Pratipanawatr W et al. Exercise training increases glycogen synthase activity and GLUT4 expression but not insulin signaling in overweight nondiabetic and type 2 diabetic subjects. Metabolism: Clinical and Experimental. 2004;53(9):1233-42.

37. Christ-Roberts CY, Pratipanawatr T, Pratipanawatr W, Berria R, Belfort R, Mandarino LJ. Increased insulin receptor signaling and glycogen synthase activity 
contribute to the synergistic effect of exercise on insulin action. Journal of Applied Physiology. 2003;95(6):2519-29.

38. Coggan AR, Swanson SC, Mendenhall LA, Habash DL, Kien CL. Effect of endurance training on hepatic glycogenolysis and gluconeogenesis during prolonged exercise in men. The American Journal of Physiology. 1995;268(3 Pt 1):E375-83.

39. Cononie CC, Goldberg AP, Rogus E, Hagberg JM. Seven consecutive days of exercise lowers plasma insulin responses to an oral glucose challenge in sedentary elderly. Journal of the American Geriatrics Society. 1994;42(4):394-8.

40. Coutinho M, Gerstein HC, Wang Y, Yusuf S. The relationship between glucose and incident cardiovascular events. A metaregression analysis of published data from 20 studies of 95,783 individuals followed for 12.4 years. Diabetes Care. 1999;22(2):233-40.

41. Dall TM, Yang W, Halder P et al. The economic burden of elevated blood glucose levels in 2012: diagnosed and undiagnosed diabetes, gestational diabetes mellitus, and prediabetes. Diabetes Care. 2014;37(12):3172-9.

42. de Oliveira VN, Bessa A, Jorge ML et al. The effect of different training programs on antioxidant status, oxidative stress, and metabolic control in type 2 diabetes. Applied Physiology, Nnutrition, and Metabolism. 2012;37(2):334-44.

43. Defronzo RA. Banting Lecture. From the triumvirate to the ominous octet: a new paradigm for the treatment of type 2 diabetes mellitus. Diabetes. 2009;58(4):77395. 
44. DeFronzo RA, Bonadonna RC, Ferrannini E. Pathogenesis of NIDDM. A balanced overview. Diabetes Care. 1992;15(3):318-68.

45. DeFronzo RA, Ferrannini E, Hendler R, Felig P, Wahren J. Regulation of splanchnic and peripheral glucose uptake by insulin and hyperglycemia in man. Diabetes. 1983;32(1):35-45.

46. DeFronzo RA, Ferrannini E, Sato Y, Felig P, Wahren J. Synergistic interaction between exercise and insulin on peripheral glucose uptake. The Journal of Clinical Investigation. 1981;68(6):1468-74.

47. DeFronzo RA, Ferrannini E, Simonson DC. Fasting hyperglycemia in noninsulin-dependent diabetes mellitus: contributions of excessive hepatic glucose production and impaired tissue glucose uptake. Metabolism: Clinical and Experimental. 1989;38(4):387-95.

48. DeFronzo RA, Tobin JD, Andres R. Glucose clamp technique: a method for quantifying insulin secretion and resistance. The American Journal of Physiology. 1979;237(3):E214-23.

49. Devlin JT, Horton ES. Effects of prior high-intensity exercise on glucose metabolism in normal and insulin-resistant men. Diabetes. 1985;34(10):973-9.

50. DiPietro L, Dziura J, Yeckel CW, Neufer PD. Exercise and improved insulin sensitivity in older women: evidence of the enduring benefits of higher intensity training. Journal of Applied Physiology. 2006;100(1):142-9.

51. Dluhy RG, McMahon GT. Intensive glycemic control in the ACCORD and ADVANCE trials. The New England Journal of Medicine. 2008;358(24):2630-3. 
52. Dunstan DW, Daly RM, Owen N et al. High-intensity resistance training improves glycemic control in older patients with type 2 diabetes. Diabetes Care. 2002;25(10):1729-36.

53. Eriksson KF, Lindgarde F. Prevention of type 2 (non-insulin-dependent) diabetes mellitus by diet and physical exercise. The 6-year Malmo feasibility study. Diabetologia. 1991;34(12):891-8.

54. Eshghi SR, Bell GJ, Boule NG. Effects of aerobic exercise with or without metformin on plasma incretins in type 2 diabetes. Canadian Journal of Ddiabetes. $2013 ; 37(6): 375-80$.

55. Ferrannini E, Bjorkman O, Reichard GA, Jr. et al. The disposal of an oral glucose load in healthy subjects. A quantitative study. Diabetes. 1985;34(6):580-8.

56. Ferrannini E, Simonson DC, Katz LD et al. The disposal of an oral glucose load in patients with non-insulin-dependent diabetes. Metabolism: Clinical and Experimental. 1988;37(1):79-85.

57. Ferrannini E, Wahren J, Felig P, DeFronzo RA. The role of fractional glucose extraction in the regulation of splanchnic glucose metabolism in normal and diabetic man. Metabolism: Clinical and Experimental. 1980;29(1):28-35.

58. Finaud J, Lac G, Filaire E. Oxidative stress : relationship with exercise and training. Sports Medicine. 2006;36(4):327-58.

59. Frayn KN. Metabolic regulation : a human perspective. 3rd ed. Chichester, U.K. ; Malden, MA: Wiley-Blackwell Pub.; 2010, xii, 371 p.

60. Frosig C, Richter EA. Improved insulin sensitivity after exercise: focus on insulin signaling. Obesity. 2009;17 Suppl 3:S15-20. 
61. Galbo H, Hedeskov CJ, Capito K, Vinten J. The effect of physical training on insulin secretion of rat pancreatic islets. Acta Physiologica Scandinavica. $1981 ; 111(1): 75-9$.

62. Gao J, Ren J, Gulve EA, Holloszy JO. Additive effect of contractions and insulin on GLUT-4 translocation into the sarcolemma. Journal of Applied Physiology. 1994;77(4):1597-601.

63. Gerich JE. Clinical significance, pathogenesis, and management of postprandial hyperglycemia. Archives of Internal Medicine. 2003;163(11):1306-16.

64. Gerstein HC, Riddle MC, Kendall DM et al. Glycemia treatment strategies in the Action to Control Cardiovascular Risk in Diabetes (ACCORD) trial. The American Journal of Cardiology. 2007;99(12A):34i-43i.

65. Gonen B, Rubenstein A, Rochman H, Tanega SP, Horwitz DL. Haemoglobin A1: An indicator of the metabolic control of diabetic patients. Lancet. $1977 ; 2(8041): 734-7$.

66. Goodyear LJ, Kahn BB. Exercise, glucose transport, and insulin sensitivity. Annu Rev Med. 1998;49:235-61.

67. Green BD, Flatt PR, Bailey CJ. Dipeptidyl peptidase IV (DPP IV) inhibitors: A newly emerging drug class for the treatment of type 2 diabetes. Diab Vasc Dis Res. 2006;3(3):159-65.

68. Health Care Cost Institute. Per capita health care spending on diabetes: 20092013. 2015. 
69. Healy GN, Dunstan DW, Salmon J et al. Objectively measured light-intensity physical activity is independently associated with 2-h plasma glucose. Diabetes Care. 2007;30(6):1384-9.

70. Heath GW, Gavin JR, 3rd, Hinderliter JM, Hagberg JM, Bloomfield SA, Holloszy JO. Effects of exercise and lack of exercise on glucose tolerance and insulin sensitivity. Journal of Applied Physiology. 1983;55(2):512-7.

71. Holm G, Bjorntorp P, Jagenburg R. Carbohydrate, lipid and amino acid metabolism following physical exercise in man. Journal of Applied Physiology: respiratory, environmental and exercise physiology. 1978;45(1):128-31.

72. Houmard JA, Shinebarger MH, Dolan PL et al. Exercise training increases GLUT-4 protein concentration in previously sedentary middle-aged men. The American Journal of Physiology. 1993;264(6 Pt 1):E896-901.

73. Howlett KF, Sakamoto K, Yu H, Goodyear LJ, Hargreaves M. Insulin-stimulated insulin receptor substrate-2-associated phosphatidylinositol 3-kinase activity is enhanced in human skeletal muscle after exercise. Metabolism: Clinical and Eexperimental. 2006;55(8):1046-52.

74. Hu FB, Sigal RJ, Rich-Edwards JW et al. Walking compared with vigorous physical activity and risk of type 2 diabetes in women: a prospective study. JAMA. 1999;282(15):1433-9.

75. Hu Y, Liu W, Chen Y et al. Combined use of fasting plasma glucose and glycated hemoglobin A1c in the screening of diabetes and impaired glucose tolerance. Acta Diabetologica. 2010;47(3):231-6. 
76. Hughes VA, Fiatarone MA, Fielding RA et al. Exercise increases muscle GLUT-4 levels and insulin action in subjects with impaired glucose tolerance. The American Journal of Pphysiology. 1993;264(6 Pt 1):E855-62.

77. Inzucchi SE, Bergenstal RM, Buse JB et al. Management of hyperglycaemia in type 2 diabetes: a patient-centered approach. Position statement of the American Diabetes Association (ADA) and the European Association for the Study of Diabetes (EASD). Diabetologia. 2012;55(6):1577-96.

78. Jenkins DJ, Ghafari H, Wolever TM et al. Relationship between rate of digestion of foods and post-prandial glycaemia. Diabetologia. 1982;22(6):450-5.

79. Jenkins DJ, Wolever TM, Taylor RH et al. Glycemic index of foods: a physiological basis for carbohydrate exchange. The American Journal of Clinical Nutrition. 1981;34(3):362-6.

80. Kahn SE. The importance of the beta-cell in the pathogenesis of type 2 diabetes mellitus. The American Journal of Medicine. 2000;108 Suppl 6a:2S-8S.

81. Kahn SE. Clinical review 135: The importance of beta-cell failure in the development and progression of type 2 diabetes. The Journal of Clinical Endocrinology and Metabolism. 2001;86(9):4047-58.

82. Kahn SE, Larson VG, Beard JC et al. Effect of exercise on insulin action, glucose tolerance, and insulin secretion in aging. The American Journal of Physiology. 1990;258(6 Pt 1):E937-43.

83. Kahn SE, Prigeon RL, McCulloch DK et al. Quantification of the relationship between insulin sensitivity and beta-cell function in human subjects. Evidence for a hyperbolic function. Diabetes. 1993;42(11):1663-72. 
84. Kang J, Robertson RJ, Hagberg JM et al. Effect of exercise intensity on glucose and insulin metabolism in obese individuals and obese NIDDM patients. Diabetes Care. 1996;19(4):341-9.

85. Karstoft K, Winding K, Knudsen SH et al. Mechanisms behind the superior effects of interval vs continuous training on glycaemic control in individuals with type 2 diabetes: a randomised controlled trial. Diabetologia. 2014;57(10):208193.

86. Katzmarzyk PT. Physical activity, sedentary behavior, and health: paradigm paralysis or paradigm shift? Diabetes. 2010;59(11):2717-25.

87. Kaufman FR. Type 2 diabetes mellitus in children and youth: a new epidemic. $J$ Pediatr Endocrinol Metab. 2002;15 Suppl 2:737-44.

88. Khunti K, Davies M, Majeed A, Thorsted BL, Wolden ML, Paul SK. Hypoglycemia and Risk of Cardiovascular Disease and All-Cause Mortality in Insulin-Treated People With Type 1 and Type 2 Diabetes: A Cohort Study. Diabetes Care. 2014.

89. Kirwan JP, Solomon TP, Wojta DM, Staten MA, Holloszy JO. Effects of 7 days of exercise training on insulin sensitivity and responsiveness in type 2 diabetes mellitus. American Journal of Physiology. Endocrinology and Metabolism. 2009;297(1):E151-6.

90. Knowler WC, Barrett-Connor E, Fowler SE et al. Reduction in the incidence of type 2 diabetes with lifestyle intervention or metformin. The New England Journal of Medicine. 2002;346(6):393-403. 
91. Kolterman OG, Buse JB, Fineman MS et al. Synthetic exendin-4 (exenatide) significantly reduces postprandial and fasting plasma glucose in subjects with type 2 diabetes. The Journal of Clinical Endocrinology and mMetabolism. 2003;88(7):3082-9.

92. Kramer FM, Jeffery RW, Forster JL, Snell MK. Long-term follow-up of behavioral treatment for obesity: patterns of weight regain among men and women. International Journal of Obesity. 1989;13(2):123-36.

93. Krogh-Madsen R, Thyfault JP, Broholm C et al. A 2-wk reduction of ambulatory activity attenuates peripheral insulin sensitivity. Journal of Applied Physiology. 2010;108(5):1034-40.

94. Lawrence RD. The Effect of Exercise on Insulin Action in Diabetes. British Medical Journal. 1926;1(3406):648-50.

95. LeBlanc J, Nadeau A, Boulay M, Rousseau-Migneron S. Effects of physical training and adiposity on glucose metabolism and 125I-insulin binding. Journal of Applied Physiology: Respiratory, Environmental and Exercise Physiology. 1979;46(2):235-9.

96. Lee YE, Kim JW, Lee EM et al. Chronic resveratrol treatment protects pancreatic islets against oxidative stress in db/db mice. PloS One. 2012;7(11):e50412.

97. Levy J, Atkinson AB, Bell PM, McCance DR, Hadden DR. Beta-cell deterioration determines the onset and rate of progression of secondary dietary failure in type 2 diabetes mellitus: the 10-year follow-up of the Belfast Diet Study. Diabetic Medicine : a Journal of the British Diabetic Association. 1998;15(4):290-6. 
98. Little JP, Gillen JB, Percival ME et al. Low-volume high-intensity interval training reduces hyperglycemia and increases muscle mitochondrial capacity in patients with type 2 diabetes. Journal of Applied Physiology. 2011;111(6):155460.

99. Lohmann D, Liebold F, Heilmann W, Senger H, Pohl A. Diminished insulin response in highly trained athletes. Metabolism: Clinical and Experimental. 1978;27(5):521-4.

100. Ludwig DS. The glycemic index: physiological mechanisms relating to obesity, diabetes, and cardiovascular disease. JAMA. 2002;287(18):2414-23.

101. Ludwig DS, Eckel RH. The glycemic index at 20 y. The American Journal of Clinical Nutrition. 2002;76(1):264S-5S.

102. Malin SK, Solomon TP, Blaszczak A, Finnegan S, Filion J, Kirwan JP. Pancreatic beta-cell function increases in a linear dose-response manner following exercise training in adults with prediabetes. American Journal of Physiology. Endocrinology and Metabolism. 2013;305(10):E1248-54.

103. Manders RJ, Van Dijk JW, van Loon LJ. Low-intensity exercise reduces the prevalence of hyperglycemia in type 2 diabetes. Medicine and Science in Sports and Exercise. 2010;42(2):219-25.

104. Mandoe MJ, Hansen KB, Hartmann B, Rehfeld JF, Holst JJ, Hansen HS. The 2monoacylglycerol moiety of dietary fat appears to be responsible for the fatinduced release of GLP-1 in humans. The American Journal of Clinical Nutrition. 2015. 
105. Martin BC, Warram JH, Krolewski AS, Bergman RN, Soeldner JS, Kahn CR. Role of glucose and insulin resistance in development of type 2 diabetes mellitus: results of a 25-year follow-up study. Lancet. 1992;340(8825):925-9.

106. Matthews DR, Cull CA, Stratton IM, Holman RR, Turner RC. UKPDS 26: Sulphonylurea failure in non-insulin-dependent diabetic patients over six years. UK Prospective Diabetes Study (UKPDS) Group. Diabetic mMedicine : a Journal of the British Diabetic Association. 1998;15(4):297-303.

107. McCarthy MI. Genomics, type 2 diabetes, and obesity. The New England Journal of Medicine. 2010;363(24):2339-50.

108. Mikus CR, Fairfax ST, Libla JL et al. Seven days of aerobic exercise training improves conduit artery blood flow following glucose ingestion in patients with type 2 diabetes. Journal of Applied Physiology. 2011;111(3):657-64.

109. Mikus CR, Oberlin DJ, Libla J, Boyle LJ, Thyfault JP. Glycaemic control is improved by 7 days of aerobic exercise training in patients with type 2 diabetes. Diabetologia. 2012;55(5):1417-23.

110. Mikus CR, Oberlin DJ, Libla JL, Taylor AM, Booth FW, Thyfault JP. Lowering physical activity impairs glycemic control in healthy volunteers. Medicine and Science in Sports and Exercise. 2012;44(2):225-31.

111. Mondon CE, Dolkas CB, Reaven GM. Site of enhanced insulin sensitivity in exercise-trained rats at rest. The American Journal of Physiology. 1980;239(3):E169-77. 
112. Monnier L, Colette C, Dunseath GJ, Owens DR. The loss of postprandial glycemic control precedes stepwise deterioration of fasting with worsening diabetes. Diabetes Care. 2007;30(2):263-9.

113. Monnier L, Colette C, Owens DR. Glycemic variability: the third component of the dysglycemia in diabetes. Is it important? How to measure it? Journal of Diabetes Science and Technology. 2008;2(6):1094-100.

114. Monnier L, Mas E, Ginet C et al. Activation of oxidative stress by acute glucose fluctuations compared with sustained chronic hyperglycemia in patients with type 2 diabetes. JAMA2006;295(14):1681-7.

115. Moss CE, Glass LL, Diakogiannaki E et al. Lipid derivatives activate GPR119 and trigger GLP-1 secretion in primary murine L-cells. Peptides. 2015.

116. Nathan DM. Long-term complications of diabetes mellitus. The New England Journal of Medicine. 1993;328(23):1676-85.

117. Nathan DM, Buse JB, Davidson MB et al. Medical management of hyperglycemia in type 2 diabetes: a consensus algorithm for the initiation and adjustment of therapy: a consensus statement of the American Diabetes Association and the European Association for the Study of Diabetes. Diabetes Care. 2009;32(1):193-203.

118. Newsholme P, Morgan D, Rebelato E et al. Insights into the critical role of NADPH oxidase(s) in the normal and dysregulated pancreatic beta cell. Diabetologia. 2009;52(12):2489-98. 
119. Nojima H, Watanabe H, Yamane K et al. Effect of aerobic exercise training on oxidative stress in patients with type 2 diabetes mellitus. Metabolism: Clinical and Experimental. 2008;57(2):170-6.

120. O'Brien JA, Shomphe LA, Kavanagh PL, Raggio G, Caro JJ. Direct medical costs of complications resulting from type 2 diabetes in the U.S. Diabetes Care. 1998;21(7):1122-8.

121. Oberlin DJ, Mikus CR, Kearney ML et al. One bout of exercise alters free-living postprandial glycemia in type 2 diabetes. Medicine and Science in Sports and Exercise. 2014;46(2):232-8.

122. Pahor M. Consideration of insurance reimbursement for physical activity and exercise programs for patients with diabetes. JAMA. 2011;305(17):1808-9.

123. Pan XR, Li GW, Hu YH et al. Effects of diet and exercise in preventing NIDDM in people with impaired glucose tolerance. The Da Qing IGT and Diabetes Study. Diabetes Care. 1997;20(4):537-44.

124. Paolisso G, D'Amore A, Galzerano D et al. Daily vitamin E supplements improve metabolic control but not insulin secretion in elderly type II diabetic patients. Diabetes Care. 1993;16(11):1433-7.

125. Poitout V, Robertson RP. Glucolipotoxicity: fuel excess and beta-cell dysfunction. Endocrinology Reviews. 2008;29(3):351-66.

126. Prasad RB, Groop L. Genetics of type 2 diabetes-pitfalls and possibilities. Genes. 2015;6(1):87-123.

127. Raciti GA, Longo M, Parrillo L et al. Understanding type 2 diabetes: from genetics to epigenetics. Acta Diabetologica. 2015. 
128. Rector RS, Thyfault JP. Does physical inactivity cause nonalcoholic fatty liver disease? Journal of Applied Physiology. 2011;111(6):1828-35.

129. Ren JM, Semenkovich CF, Gulve EA, Gao J, Holloszy JO. Exercise induces rapid increases in GLUT4 expression, glucose transport capacity, and insulin-stimulated glycogen storage in muscle. Journal of Biological Chemistry. 1994;269(20):14396-401.

130. Reynolds LJ, Credeur DP, Holwerda SW, Leidy HJ, Fadel PJ, Thyfault JP. Acute Inactivity Impairs Glycemic Control but Not Blood Flow to Glucose Ingestion. Medicine and Science in Sports and Exercise. 2014.

131. Richard D, LeBlanc J. Effects of physical training and food restriction on insulin secretion and glucose tolerance in male and female rats. The American Journal of Clinical Nutrition. 1980;33(12):2588-94.

132. Richter EA, Garetto LP, Goodman MN, Ruderman NB. Muscle glucose metabolism following exercise in the rat: increased sensitivity to insulin. The Journal of Clinical Investigation. 1982;69(4):785-93.

133. Richter EA, Mikines KJ, Galbo H, Kiens B. Effect of exercise on insulin action in human skeletal muscle. Journal of Applied Physiology. 1989;66(2):876-85.

134. Rijkelijkhuizen JM, Girman CJ, Mari A et al. Classical and model-based estimates of beta-cell function during a mixed meal vs. an OGTT in a populationbased cohort. Diabetes Research and Clinical Practice. 2009;83(2):280-8.

135. Robertson RP. Chronic oxidative stress as a central mechanism for glucose toxicity in pancreatic islet beta cells in diabetes. Journal of Biological Chemistry. 2004;279(41):42351-4. 
136. Robertson RP, Harmon J, Tran PO, Poitout V. Beta-cell glucose toxicity, lipotoxicity, and chronic oxidative stress in type 2 diabetes. Diabetes. 2004;53 Suppl 1:S119-24.

137. Robertson RP, Harmon JS. Diabetes, glucose toxicity, and oxidative stress: A case of double jeopardy for the pancreatic islet beta cell. Free Radic Biol Med. 2006;41(2):177-84.

138. Rockl KS, Witczak CA, Goodyear LJ. Signaling mechanisms in skeletal muscle: acute responses and chronic adaptations to exercise. IUBMB Life. 2008;60(3):145-53.

139. Rogers MA, Yamamoto C, King DS, Hagberg JM, Ehsani AA, Holloszy JO. Improvement in glucose tolerance after $1 \mathrm{wk}$ of exercise in patients with mild NIDDM. Diabetes Care. 1988;11(8):613-8.

140. Rohlfing CL, Wiedmeyer HM, Little RR, England JD, Tennill A, Goldstein DE. Defining the relationship between plasma glucose and $\mathrm{HbA}(1 \mathrm{c})$ : analysis of glucose profiles and $\mathrm{HbA}(1 \mathrm{c})$ in the Diabetes Control and Complications Trial. Diabetes Care. 2002;25(2):275-8.

141. Rynders CA, Weltman JY, Jiang B et al. Effects of exercise intensity on postprandial improvement in glucose disposal and insulin sensitivity in prediabetic adults. The Journal of Clinical Endocrinology and Metabolism. 2014;99(1):220-8.

142. Saha S, Gerdtham UG, Johansson P. Economic evaluation of lifestyle interventions for preventing diabetes and cardiovascular diseases. International Journal of Environmental Research and Public Health. 2010;7(8):3150-95. 
143. Saydah SH, Miret M, Sung J, Varas C, Gause D, Brancati FL. Postchallenge hyperglycemia and mortality in a national sample of U.S. adults. Diabetes Care. 2001;24(8):1397-402.

144. Sigal RJ, Kenny GP, Boule NG et al. Effects of aerobic training, resistance training, or both on glycemic control in type 2 diabetes: a randomized trial. Annals of Internal Medicine. 2007;147(6):357-69.

145. Singer DE, Nathan DM, Anderson KM, Wilson PW, Evans JC. Association of HbA1c with prevalent cardiovascular disease in the original cohort of the Framingham Heart Study. Diabetes. 1992;41(2):202-8.

146. Singh S, Loke YK, Furberg CD. Long-term risk of cardiovascular events with rosiglitazone: a meta-analysis. JAMA. 2007;298(10):1189-95.

147. Skyler JS, Bergenstal R, Bonow RO et al. Intensive glycemic control and the prevention of cardiovascular events: implications of the ACCORD, ADVANCE, and VA diabetes trials: a position statement of the American Diabetes Association and a scientific statement of the American College of Cardiology Foundation and the American Heart Association. Diabetes Care. 2009;32(1):187-92.

148. Slentz CA, Tanner CJ, Bateman LA et al. Effects of exercise training intensity on pancreatic beta-cell function. Diabetes Care. 2009;32(10):1807-11.

149. Solomon TP, Haus JM, Kelly KR et al. A low-glycemic index diet combined with exercise reduces insulin resistance, postprandial hyperinsulinemia, and glucosedependent insulinotropic polypeptide responses in obese, prediabetic humans. The American Journal of Clinical Nutrition. 2010;92(6):1359-68. 
150. Solomon TP, Malin SK, Karstoft K, Kashyap SR, Haus JM, Kirwan JP.

Pancreatic beta-cell function is a stronger predictor of changes in glycemic control after an aerobic exercise intervention than insulin sensitivity. The Journal of Clinical Endocrinology and Metabolism. 2013;98(10):4176-86.

151. Solomon TP, Malin SK, Karstoft K et al. Determining pancreatic beta-cell compensation for changing insulin sensitivity using an oral glucose tolerance test. American Journal of Physiology. Endocrinology and Metabolism. 2014;307(9):E822-9.

152. Solomon TP, Thyfault JP. Type 2 diabetes sits in a chair. Diabetes, Obesity \& Metabolism. 2013;15(11):987-92.

153. Stettler C, Allemann S, Juni P et al. Glycemic control and macrovascular disease in types 1 and 2 diabetes mellitus: Meta-analysis of randomized trials. American Heart Journal. 2006;152(1):27-38.

154. Stratton IM, Adler AI, Neil HA et al. Association of glycaemia with macrovascular and microvascular complications of type 2 diabetes (UKPDS 35): prospective observational study. British Medical Journal. 2000;321(7258):40512.

155. Temelkova-Kurktschiev TS, Koehler C, Henkel E, Leonhardt W, Fuecker K, Hanefeld M. Postchallenge plasma glucose and glycemic spikes are more strongly associated with atherosclerosis than fasting glucose or $\mathrm{HbA1c}$ level. Diabetes Care. 2000;23(12):1830-4. 
156. The ADVANCE Collaborative Group. Intensive blood glucose control and vascular outcomes in patients with type 2 diabetes. The New England Journal of Medicine. 2008;358(24):2560-72.

157. The Look AHEAD Research Group. Long-term effects of a lifestyle intervention on weight and cardiovascular risk factors in individuals with type 2 diabetes mellitus: four-year results of the Look AHEAD trial. Archives of Internal Medicine. 2010;170(17):1566-75.

158. Thomas DE, Elliott EJ, Naughton GA. Exercise for type 2 diabetes mellitus. The Cochrane Database of Systematic Reviews. 2006;(3):CD002968.

159. Thyfault JP. Setting the stage: possible mechanisms by which acute contraction restores insulin sensitivity in muscle. American Journal of Physiology. Regulatory, Integrative and Comparative Physiology. 2008;294(4):R1103-10.

160. Thyfault JP, Booth FW. Lack of regular physical exercise or too much inactivity. Current Opinion in Clinical Nutrition and mMetabolic Care. 2011;14(4):374-8.

161. Thyfault JP, Du M, Kraus WE, Levine JA, Booth FW. Physiology of sedentary behavior and its relationship to health outcomes. Medicine and Science in Sports and Exercise. 2014;47(6):1301-5.

162. Thyfault JP, Krogh-Madsen R. Metabolic disruptions induced by reduced ambulatory activity in free-living humans. Journal of Applied Physiology. 2011;111(4):1218-24.

163. Todd JF, Bloom SR. Incretins and other peptides in the treatment of diabetes. Diabetic Medicine : a Journal of the British Diabetic Association. 2007;24(3):223-32. 
164. Tominaga M, Eguchi H, Manaka H, Igarashi K, Kato T, Sekikawa A. Impaired glucose tolerance is a risk factor for cardiovascular disease, but not impaired fasting glucose. The Funagata Diabetes Study. Diabetes Care. 1999;22(6):920-4.

165. Traub ML, Jain A, Maslow BS et al. The "muffin test"--an alternative to the oral glucose tolerance test for detecting impaired glucose tolerance. Menopause. 2012;19(1):62-6.

166. Tuomilehto J, Lindstrom J, Eriksson JG et al. Prevention of type 2 diabetes mellitus by changes in lifestyle among subjects with impaired glucose tolerance. The New England Journal of Medicine. 2001;344(18):1343-50.

167. Turner RC. The U.K. Prospective Diabetes Study. A review. Diabetes Care. 1998;21 Suppl 3:C35-8.

168. Turner RC, Cull CA, Frighi V, Holman RR. Glycemic control with diet, sulfonylurea, metformin, or insulin in patients with type 2 diabetes mellitus: progressive requirement for multiple therapies (UKPDS 49). UK Prospective Diabetes Study (UKPDS) Group. JAMA. 1999;281(21):2005-12.

169. Umpierre D, Ribeiro PA, Kramer CK et al. Physical activity advice only or structured exercise training and association with HbA1c levels in type 2 diabetes: a systematic review and meta-analysis. JAMA. 2011;305(17):1790-9.

170. Utzschneider KM, Prigeon RL, Faulenbach MV et al. Oral disposition index predicts the development of future diabetes above and beyond fasting and 2-h glucose levels. Diabetes Care. 2009;32(2):335-41.

171. Wadley GD, Konstantopoulos N, Macaulay L et al. Increased insulin-stimulated Akt pSer473 and cytosolic SHP2 protein abundance in human skeletal muscle 
following acute exercise and short-term training. Journal of Applied Physiology. 2007;102(4):1624-31.

172. Wagenknecht LE, D'Agostino RB, Jr., Haffner SM, Savage PJ, Rewers M. Impaired glucose tolerance, type 2 diabetes, and carotid wall thickness: the Insulin Resistance Atherosclerosis Study. Diabetes Care. 1998;21(11):1812-8.

173. Weiss EP, Royer NK, Fisher JS, Holloszy JO, Fontana L. Postprandial plasma incretin hormones in exercise-trained versus untrained subjects. Medicine and Science in Sports and Exercise. 2014;46(6):1098-103.

174. Wing RR, Marcus MD, Epstein LH, Salata R. Type II diabetic subjects lose less weight than their overweight nondiabetic spouses. Diabetes Care. 1987;10(5):563-6.

175. Woerle HJ, Szoke E, Meyer C et al. Mechanisms for abnormal postprandial glucose metabolism in type 2 diabetes. American Journal of Physiology. Endocrinology and Metabolism. 2006;290(1):E67-E77.

176. Wojtaszewski JF, Richter EA. Effects of acute exercise and training on insulin action and sensitivity: focus on molecular mechanisms in muscle. Essays in Biochemistry. 2006;42:31-46.

177. Wolever TM, Chiasson JL, Csima A et al. Variation of postprandial plasma glucose, palatability, and symptoms associated with a standardized mixed test meal versus 75 g oral glucose. Diabetes Care. 1998;21(3):336-40.

178. Wolever TM, Jenkins DJ. The use of the glycemic index in predicting the blood glucose response to mixed meals. The American Journal of Clinical Nutrition. 1986;43(1):167-72. 
179. Wolever TM, Yang M, Zeng XY, Atkinson F, Brand-Miller JC. Food glycemic index, as given in glycemic index tables, is a significant determinant of glycemic responses elicited by composite breakfast meals. The American Journal of Clinical Nutrition. 2006;83(6):1306-12.

180. $\mathrm{Xu} \mathrm{G}, \mathrm{Li} \mathrm{Z}$, Ding L et al. Intestinal mTOR regulates GLP-1 production in mouse L cells. Diabetologia. 2015;58(8):1887-97.

181. Zierath JR. Invited review: Exercise training-induced changes in insulin signaling in skeletal muscle. Journal of Applied Physiology. 2002;93(2):773-81. 


\section{VITA}

Monica L. Kearney earned a Bachelor of Science degree in Health Management with an emphasis in Fitness and Sports Medicine and a minor in Nutrition from Southeast Missouri State University in 2005, where she graduated magna cum laude. She then spent time employed in community fitness and corporate wellness before returning to Southeast Missouri State University, where she earned a Master of Science degree in Nutrition and Exercise Science in 2009. Monica continued her graduate education at the University of Missouri under the mentorship of Dr. Tom Thomas and Dr. John Thyfault, where she will earn her doctorate in Exercise Physiology in 2015. Monica is an avid believer in the power of movement and physical activity for maintaining and improving all aspects of health and wellness. She has accepted a postdoctoral research fellowship with Washington University School of Medicine, in St. Louis. 